\title{
NORMAL APPROXIMATION FOR STABILIZING FUNCTIONALS ${ }^{1}$
}

\author{
By RAPHAËL LACHIÈZE-REY*, MATthiAs SCHULTE ${ }^{\dagger}$ AND J. E. YUKICH \\ Université Paris Descartes, Sorbonne Paris Cité*, Universität Bern ${ }^{\dagger}$ \\ and Lehigh University
}

We establish presumably optimal rates of normal convergence with respect to the Kolmogorov distance for a large class of geometric functionals of marked Poisson and binomial point processes on general metric spaces. The rates are valid whenever the geometric functional is expressible as a sum of exponentially stabilizing score functions satisfying a moment condition. By incorporating stabilization methods into the Malliavin-Stein theory, we obtain rates of normal approximation for sums of stabilizing score functions which either improve upon existing rates or are the first of their kind.

Our general rates hold for functionals of marked input on spaces more general than full-dimensional subsets of $\mathbb{R}^{d}$, including $m$-dimensional Riemannian manifolds, $m \leq d$. We use the general results to deduce improved and new rates of normal convergence for several functionals in stochastic geometry, including those whose variances re-scale as the volume or the surface area of an underlying set. In particular, we improve upon rates of normal convergence for the $k$-face and $i$ th intrinsic volume functionals of the convex hull of Poisson and binomial random samples in a smooth convex body in dimension $d \geq 2$. We also provide improved rates of normal convergence for statistics of nearest neighbors graphs and high-dimensional data sets, the number of maximal points in a random sample, estimators of surface area and volume arising in set approximation via Voronoi tessellations, and clique counts in generalized random geometric graphs.

\section{CONTENTS}

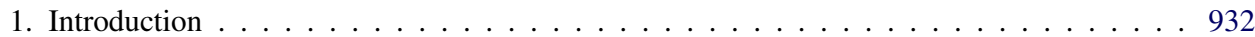

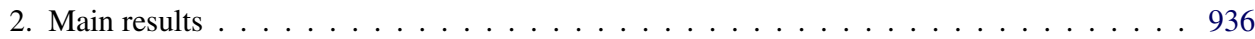

2.1. Notation and definitions . . . . . . . . . . . . . . . . . 936

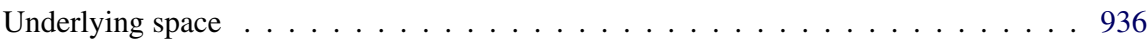

Marked Poisson and binomial point processes _ . . . . . . . . . . . 937

Score functions . . . . . . . . . . . . . . . . . . . . . . . . . 937

2.2. Statements of the main results _ . . . . . . . . . . . . . . . . . . . . 940

3. Applications . . . . . . . . . . . . . . . . . . . . . . . . . . 944

3.1. Nearest neighbors graphs and statistics of high-dimensional data sets . . . . . . . . . 945

Received December 2017; revised May 2018.

${ }^{1}$ Supported in part by NSF Grant DMS-14-06410 and the DFG research unit "Geometry and Physics of Spatial Random Systems".

MSC2010 subject classifications. Primary 60F05; secondary 60D05.

Key words and phrases. Stein's method, Malliavin calculus, stabilization, random Euclidean graphs, statistics of data sets, statistics of convex hulls, Voronoi set approximation, maximal points. 
3.2. Maximal points . . . . . . . . . . . . . . . . . . . . . . . . . . . 949

3.3. Set approximation via Voronoi tessellations . . . . . . . . . . . . . . 952

3.4. Statistics of convex hulls of random point samples . . . . . . . . . . . . . . 956

3.5. Clique counts in generalized random geometric graphs . . . . . . . . . . . . . . . 965

4. Malliavin-Stein bounds . . . . . . . . . . . . . . . . . . . 968

5. Proofs of Theorem 2.1 and Theorem $2.3 \ldots \ldots \ldots 7 \ldots$

Appendix . . . . . . . . . . . . . . . . . . . . . . . . . . . . 990

Acknowledgments . . . . . . . . . . . . . . . . . . . . . . . 991

References . . . . . . . . . . . . . . . . . . . . . . . 991

1. Introduction. Let $(\mathbb{X}, \mathcal{F})$ be a measurable space equipped with a $\sigma$-finite measure $\mathbb{Q}$ and a measurable semi-metric $\mathrm{d}: \mathbb{X} \times \mathbb{X} \rightarrow[0, \infty)$. For all $s \geq 1$, let $\mathcal{P}_{s}$ be a Poisson point process with intensity measure $s \mathbb{Q}$. When $\mathbb{Q}$ is a probability measure, we let $\mathcal{X}_{n}$ be a binomial point process of $n$ points which are i.i.d. according to $\mathbb{Q}$. Consider the statistics

$$
H_{s}:=h_{s}\left(\mathcal{P}_{s}\right):=\sum_{x \in \mathcal{P}_{s}} \xi_{s}\left(x, \mathcal{P}_{s}\right), \quad s \geq 1,
$$

and

$$
H_{n}^{\prime}:=h_{n}\left(\mathcal{X}_{n}\right):=\sum_{x \in \mathcal{X}_{n}} \xi_{n}\left(x, \mathcal{X}_{n}\right), \quad n \in \mathbb{N},
$$

where, roughly speaking, the scores $\xi_{s}\left(x, \mathcal{P}_{s}\right)$ and $\xi_{n}\left(x, \mathcal{X}_{n}\right)$ represent the local contributions to the global statistics $H_{s}$ and $H_{n}^{\prime}$, respectively. Functionals such as $H_{s}$ and $H_{n}^{\prime}$, which are in some sense locally defined, are called stabilizing functionals. The concept of stabilization and the systematic investigation of stabilizing functionals go back to the papers [28, 29]. In the following, we are interested in quantitative central limit theorems for stabilizing functionals, whereas laws of large numbers are shown in [26, 29] and moderate deviations are considered in [13]. For a survey on limit theorems in stochastic geometry with a particular focus on stabilization, we refer to [40]. Statistics $H_{s}$ and $H_{n}^{\prime}$ typically describe a global property of a random geometric structure on $\mathbb{X}$ in terms of local contributions exhibiting spatial interaction and dependence. Functionals in stochastic geometry which may be cast in the form of (1.1) and (1.2) include total edge length and clique counts in random graphs, statistics of Voronoi set approximation, the $k$-face and volume functional of convex hulls of random point samples, as well as statistics of RSA packing models and spatial birth growth models.

In the following, we allow that the underlying point processes $\mathcal{P}_{s}$ and $\mathcal{X}_{n}$ are marked, that is, that an i.i.d. random mark is attached to each of their points.

Throughout this paper, we denote by $N$ a standard Gaussian random variable and by

$$
d_{K}(Y, Z):=\sup _{t \in \mathbb{R}}|\mathbb{P}(Y \leq t)-\mathbb{P}(Z \leq t)|
$$


the Kolmogorov distance of two random variables $Y$ and $Z$. For a sum $S_{n}=$ $\sum_{i=1}^{n} Y_{i}$ of $n$ i.i.d. random variables $Y_{1}, \ldots, Y_{n}$ such that $\mathbb{E}\left|Y_{1}\right|^{3}<\infty$, it is known from the classical Berry-Esseen theorem that

$$
d_{K}\left(\frac{S_{n}-\mathbb{E} S_{n}}{\sqrt{\operatorname{Var} S_{n}}}, N\right) \leq \frac{C \mathbb{E}\left|Y_{1}-\mathbb{E} Y_{1}\right|^{3}}{\operatorname{Var} Y_{1}} \frac{1}{\sqrt{\operatorname{Var} S_{n}}}, \quad n \in \mathbb{N},
$$

with $C \in(0, \infty)$ a universal constant. By considering special choices for $Y_{1}, \ldots$, $Y_{n}$, one can show that the rate $1 / \sqrt{\operatorname{Var} S_{n}}$ in (1.4) is optimal. The main contribution of this paper is to show that exponentially stabilizing functionals $H_{s}$ and $H_{n}^{\prime}$ satisfy bounds resembling those at (1.4), with rates $1 / \sqrt{\operatorname{Var} H_{s}}$ and $1 / \sqrt{\operatorname{Var} H_{n}^{\prime}}$, respectively. Here, the scores $\left(\xi_{s}\right)_{s \geq 1}$ and $\left(\xi_{n}\right)_{n \in \mathbb{N}}$ have uniformly bounded $(4+p)$ th moments for some $p>0$, similar to the assumption $\mathbb{E}\left|Y_{1}\right|^{3}<\infty$ at (1.4). In contrast to the summands of $S_{n}$, the summands of $H_{s}$ and $H_{n}^{\prime}$ are dependent in general, but nevertheless by comparison with the classical Berry-Esseen theorem, one can expect that the rates $1 / \sqrt{\operatorname{Var} H_{s}}$ and $1 / \sqrt{\operatorname{Var} H_{n}^{\prime}}$ are optimal. Thus, whenever such rates occur, we say in the sequel that they are presumably optimal. In order to show that the rates are indeed optimal, one would need to derive lower bounds for the Kolmogorov distance of the same order. This seems to be a completely different problem, which, as far as we know, is open and presumably difficult, as the scores comprising the sums $H_{s}$ and $H_{n}^{\prime}$ have a nontrivial dependence structure.

In stochastic geometry, it is frequently the case that $\left(H_{S}-\mathbb{E} H_{S}\right) / \sqrt{\operatorname{Var} H_{S}}$ converges to the standard normal, and likewise for $\left(H_{n}^{\prime}-\mathbb{E} H_{n}^{\prime}\right) / \sqrt{\operatorname{Var} H_{n}^{\prime}}$. However, up to now there has been no systematic treatment which establishes presumably optimal rates of convergence to the normal. For example, in [8] a central limit theorem for functionals of nearest neighbor graphs is derived, but no rate of convergence is given. Dependency graph methods are used in [1] to show asymptotic normality of the total edge length of the nearest neighbor graph as well as of the Voronoi and Delaunay tessellations, but they lead to suboptimal rates of convergence. Anticipating stabilization methods, the authors of [19] proved asymptotic normality for the total edge length of the Euclidean minimal spanning tree, though they did not obtain a rate of convergence. In the papers [7, 25, 28], abstract central limit theorems for stabilizing functionals are derived and applied to several problems from stochastic geometry. Quantitative bounds for the normal approximation of stabilizing functionals of an underlying Poisson point process are given in $[5,27,30,31,48]$. These results yield rates of convergence for the Kolmogorov distance of the order $1 / \sqrt{\operatorname{Var} H_{S}}$ times some extraneous logarithmic factors. For stabilizing functionals of an underlying binomial point process, we are unaware of analogous results. The paper [10] uses Stein's method to provide rates of normal convergence for functionals on binomial input satisfying a type of local dependence, though these rates are in the Wasserstein distance.

Recent work [22] shows that the Malliavin calculus, combined with Stein's method of normal approximation, yields rates of normal approximation for general 
Poisson functionals. The rates are in the Kolmogorov distance, they are presumably optimal, and the authors use their general results to deduce rates of normal convergence (cf. Proposition 1.4 and Theorem 6.1 of [22]) for Poisson functionals satisfying a type of stabilization. That paper states that "the new connection between the Stein-Malliavin approach and the theory of stabilization has a great potential for further generalisations and applications", though it stops short of linking these two fertile research areas.

The first main goal of this paper is to fully develop this connection, showing that the theory of stabilization neatly dovetails with Malliavin-Stein methods, giving presumably optimal rates of normal convergence. Malliavin-Stein bounds for normal approximation, expressed in terms of moments of first- and second-order difference operators [22], seemingly consist of unwieldy terms. However, if $\xi_{s}$ is exponentially stabilizing and satisfies a moment condition, then our first main goal is to show that the Malliavin-Stein bounds remarkably simplify, showing that

$$
d_{K}\left(\frac{H_{s}-\mathbb{E} H_{s}}{\sqrt{\operatorname{Var} H_{s}}}, N\right) \leq \frac{\tilde{C}}{\sqrt{\operatorname{Var} H_{s}}}, \quad s \geq 1,
$$

as explained in Corollary 2.2. These rates, presumed optimal, remove extraneous logarithmic factors appearing in [5, 27, 30, 31, 48].

Our second main goal is to show that (1.5) holds when $H_{s}$ is replaced by $H_{n}^{\prime}$, thus giving analogous rates of normal convergence when Poisson input is replaced by binomial input. Recall that the paper [20] (see Theorem 5.1 there) uses Stein's method and difference operators to establish rates of normal convergence in the Kolmogorov distance for general functionals of binomial point processes. Though [20] deduces rates of normal convergence for some statistics of binomial input in geometric probability, it too stops short of systematically developing the connection between stabilization, Stein's method and difference operators. Our second goal is to explicitly and fully develop this connection. As a by-product, we show that the ostensibly unmanageable bounds in the Kolmogorov distance may be recast into bounds which collapse into a single term $1 / \sqrt{\operatorname{Var} H_{n}^{\prime}}$. In other words, when $\xi_{n}$ has a a radius of stabilization (with respect to binomial input $\mathcal{X}_{n}$ ) which decays exponentially fast, then subject to a moment condition on $\xi_{n}$, Corollary 2.2 shows

$$
d_{K}\left(\frac{H_{n}^{\prime}-\mathbb{E} H_{n}^{\prime}}{\sqrt{\operatorname{Var} H_{n}^{\prime}}}, N\right) \leq \frac{\tilde{C}^{\prime}}{\sqrt{\operatorname{Var} H_{n}^{\prime}}}, \quad n \geq 9 .
$$

The main finding of this paper, culminating much research related to stabilizing score functionals and captured by the rate results (1.5) and (1.6), is this: Statistics (1.1) and (1.2) enjoy presumably optimal rates of normal convergence once the scores $\xi_{s}$ and $\xi_{n}$ satisfy exponential stabilization and a moment condition. In problems of interest, the verification of these conditions is sometimes a straightforward exercise, as seen in Section 3, the applications section. On the other hand, 
for statistics involving convex hulls of random point samples in a smooth compact convex set, the verification of these conditions involves a judicious choice of the underlying metric space, one which allows us to express complicated spatial dependencies in a relatively simple fashion. This is all illustrated in Section 3.4, where it is shown for both the intrinsic volumes of the convex hull and for the counts of its lower dimensional faces, that the convergence rates (1.5) and (1.6) are either the first of their kind or that they improve upon existing rates of convergence in the literature, for both Poisson and binomial input in all dimensions $d \geq 2$.

Our third and final goal is to broaden the scope of existing central limit theory in such a way that:

(i) The presumably optimal rates (1.5) and (1.6) are applicable both in the context of volume order and of surface area order scaling of the variance of the functional. By this, we mean that the variance of $H_{s}$ (resp., $H_{n}^{\prime}$ ) is of order $s$ (resp., $n$ ) or $s^{1-1 / d}$ (resp., $n^{1-1 / d}$ ), after renormalising so that the score of an arbitrary point is of constant order. The notions volume order scaling and surface area order scaling come from a different (but for many problems equivalent) formulation where the intensity of the underlying point process is kept fixed and a set carrying the input is dilated instead. In this set-up, the variance may be asymptotically proportional to the volume or surface area of the carrying set. Surface order scaling of the variance typically arises when the scores are nonvanishing only for points close to a $(d-1)$-dimensional subset of $\mathbb{R}^{d}$. As shown in Theorems 3.3 and 3.4 , this generality yields improved rates of normal convergence for the number of maximal points in a random sample and for statistics arising in Voronoi set approximation, respectively.

(ii) The methods are sufficiently general so that they bring within their purview score functions of data on spaces $(\mathbb{X}, \mathrm{d})$, with $\mathrm{d}$ an arbitrary semi-metric. We illustrate the power of our general approach by establishing a self-contained, relatively short proof of the asymptotic normality of statistics of convex hulls of random point samples as discussed earlier in this Introduction. Our methods also deliver rates of convergence for statistics of $k$-nearest neighbors graphs and clique counts on both Poisson and binomial input on general metric spaces $(\mathbb{X}, d)$, as seen in Theorems 3.1 and 3.15.

We anticipate that the generality of the methods here will lead to further nontrivial applications in the central limit theory for functionals in stochastic geometry.

This paper is organized as follows. In Section 2, we give general bounds for the normal approximation of stabilizing functionals of Poisson or binomial input, which are our main theoretical findings. In Section 3, we demonstrate the power of our general bounds by deducing rates of normal approximation for several problems from stochastic geometry. We show in Section 4 that some existing Malliavin-Stein bounds [20, 22] may be cast into similar forms facilitating normal approximation of sums of stabilizing scores of Poisson and binomial input. Section 5 combines these bounds with moment and probability bounds for first- and second-order difference operators to prove our main theoretical results. 


\section{Main results.}

2.1. Notation and definitions. Before we present our main results in the next subsection, we fix our framework by introducing some notation and definitions. We spell out the assumptions on the underlying space, define marked Poisson and binomial point processes, and introduce several crucial properties of the score functions comprising $H_{S}$ and $H_{n}^{\prime}$ defined at (1.1) and (1.2).

Underlying space. Let $(\mathbb{X}, \mathcal{F})$ be a measurable space with a $\sigma$-finite measure $\mathbb{Q}$ and a measurable semi-metric $\mathrm{d}: \mathbb{X} \times \mathbb{X} \rightarrow[0, \infty)$, that is, in contrast to the definition of a metric, one allows $\mathrm{d}(x, y)=0$ for distinct $x, y \in \mathbb{X}$. By $B(x, r)$ we denote the ball of radius $r \geq 0$ around $x \in \mathbb{X}$, that is, $B(x, r):=\{y \in \mathbb{X}: \mathrm{d}(x, y) \leq$ $r$. Note that the measurability of $\mathrm{d}$ implies that all these balls belong to $\mathcal{F}$. In the standard set-up for stabilizing functionals, $\mathbb{X}$ is a subset of $\mathbb{R}^{d}$ and $\mathbb{Q}$ has a bounded density with respect to the Lebesgue measure (see, e.g., [25, 30, 48]). To handle more general $\mathbb{X}$ and $\mathbb{Q}$, we replace this standard assumption by the following growth condition on the $\mathbb{Q}$-surface area of spheres: There are constants $\gamma, \kappa>0$ such that

$$
\limsup _{\varepsilon \rightarrow 0} \frac{\mathbb{Q}(B(x, r+\varepsilon))-\mathbb{Q}(B(x, r))}{\varepsilon} \leq \kappa \gamma r^{\gamma-1}, \quad r \geq 0, x \in \mathbb{X} .
$$

This assumption implies that $\mathbb{Q}$ is diffuse, that is, $\mathbb{Q}(\{x\})=0$ for $x \in \mathbb{X}$ [see Lemma 5.1(a)].

Two examples for measure spaces $(\mathbb{X}, \mathcal{F}, \mathbb{Q})$ and semi-metrics $d$ satisfying the assumption (2.1) are the following:

- Example 1. Let $\mathbb{X}$ be a full-dimensional subset of $\mathbb{R}^{d}$ equipped with the induced Borel- $\sigma$-field $\mathcal{F}$ and the usual Euclidean distance d, assume that $\mathbb{Q}$ is a measure on $\mathbb{X}$ with a density $g$ with respect to the Lebesgue measure, and put $\gamma:=d$. Then condition (2.1) reduces to the standard assumption that $g$ is bounded. Indeed, if $\|g\|_{\infty}:=\sup _{x \in \mathbb{X}}|g(x)|<\infty$, then (2.1) is obviously satisfied with $\kappa:=\|g\|_{\infty} \kappa_{d}$, where $\kappa_{d}:=\pi^{d / 2} / \Gamma(d / 2+1)$ is the volume of the $d$-dimensional unit ball in $\mathbb{R}^{d}$. On the other hand, if (2.1) holds, then $\mathbb{Q}(B(x, r)) \leq \kappa r^{d}$ as seen by Lemma 5.1(a) below. This gives an upper bound of $\kappa / \kappa_{d}$ for $g$ since, by Lebesgue's differentiation theorem, Lebesgue almost all points $x$ in $\mathbb{R}^{d}$ are Lebesgue points, that is to say

$$
g(x)=\lim _{r \rightarrow 0}\left(\kappa_{d} r^{d}\right)^{-1} \int_{y \in B(x, r)} g(y) \mathrm{d} y=\lim _{r \rightarrow 0}\left(\kappa_{d} r^{d}\right)^{-1} \mathbb{Q}(B(x, r)) \leq \kappa / \kappa_{d} .
$$

- Example 2. Let $\mathbb{X} \subseteq \mathbb{R}^{d}$ be a smooth $m$-dimensional subset of $\mathbb{R}^{d}, m \leq d$, equipped with a semi-metric $d$, and a measure $\mathbb{Q}$ on $\mathbb{X}$ with a bounded density $g$ with respect to the uniform surface measure $\operatorname{Vol}_{m}$ on $\mathbb{X}$. We assume that 
the $\operatorname{Vol}_{m-1}$ measure of the sphere $\partial(B(x, r))$ is bounded by the surface area of the Euclidean sphere $\mathbb{S}^{m-1}(0, r)$ of the same radius, that is to say

$$
\operatorname{Vol}_{m-1}(\partial B(x, r)) \leq m \kappa_{m} r^{m-1}, \quad x \in \mathbb{X}, r>0 .
$$

When $\mathbb{X}$ is an $m$-dimensional affine space and $\mathrm{d}$ is the usual Euclidean metric on $\mathbb{R}^{d},(2.2)$ holds with equality, naturally. However (2.2) holds in more general situations. For example, by Bishop's comparison theorem (Theorem 1.2 of [39], along with (1.15) there), (2.2) holds for Riemannian manifolds $\mathbb{X}$ with nonnegative Ricci curvature, with $d$ the geodesic distance. Given the bound (2.2), one obtains (2.1) with $\kappa=\|g\|_{\infty} \kappa_{m}$ and $\gamma=m$. This example includes the case $\mathbb{X}=\mathbb{S}^{m}$, the unit sphere in $\mathbb{R}^{m+1}$ equipped with the geodesic distance.

Marked Poisson and binomial point processes. To deal with marked point processes, let $\left(\mathbb{M}, \mathcal{F}_{\mathbb{M}}, \mathbb{Q}_{\mathbb{M}}\right)$ be a probability space. In the following, $\mathbb{M}$ shall be the space of marks and $\mathbb{Q}_{\mathbb{M}}$ the underlying probability measure of the marks. Let $\widehat{\mathbb{X}}:=\mathbb{X} \times \mathbb{M}$, put $\widehat{\mathcal{F}}$ to be the product $\sigma$-field of $\mathcal{F}$ and $\mathcal{F}_{\mathbb{M}}$, and let $\widehat{\mathbb{Q}}$ be the product measure of $\mathbb{Q}$ and $\mathbb{Q}_{\mathbb{M}}$. When $\left(\mathbb{M}, \mathcal{F}_{\mathbb{M}}, \mathbb{Q}_{\mathbb{M}}\right)$ is a singleton endowed with a Dirac point mass, $\widehat{\mathbb{X}}$ reduces to $\mathbb{X}$ and the "hat" superscript can be removed in all occurrences.

Let $\mathbf{N}$ be the set of all counting measures $v$ on $\widehat{\mathbb{X}}$ that are simple [i.e., $v(\{\hat{x}\}) \leq 1$ for all $\hat{x} \in \widehat{\mathbb{X}}]$ and locally finite [i.e., $v(B)<\infty$ for all bounded $B \in \widehat{\mathcal{F}}$ ]. These counting measures can be interpreted as point configurations in $\widehat{\mathbb{X}}$. Thus we treat the elements from $\mathbf{N}$ as sets in our notation. The set $\mathbf{N}$ is equipped with the smallest $\sigma$-field $\mathcal{N}$ such that the maps $m_{A}: \mathbf{N} \rightarrow \mathbb{N} \cup\{0, \infty\}, \mathcal{M} \mapsto \mathcal{M}(A)$ are measurable for all $A \in \widehat{\mathcal{F}}$. A point process is now a random element in $\mathbf{N}$. In this paper, we consider two different classes of point processes, namely Poisson and binomial point processes. For $s \geq 1$, update the notation $\mathcal{P}_{s}$ to represent a Poisson point process with intensity measure $s \widehat{\mathbb{Q}}$. This means that the numbers of points of $\mathcal{P}_{s}$ in disjoint sets $A_{1}, \ldots, A_{m} \in \widehat{\mathcal{F}}, m \in \mathbb{N}$, are independent and that the number of points of $\mathcal{P}_{s}$ in a set $A \in \widehat{\mathcal{F}}$ follows a Poisson distribution with mean $s \widehat{\mathbb{Q}}(A)$. For a comprehensive treatment of Poisson point processes, we refer to the monograph [23]. In case $\mathbb{Q}$ is a probability measure, we denote similarly by $\mathcal{X}_{n}$ a binomial point process of $n \in \mathbb{N}$ points that are independently distributed according to $\widehat{\mathbb{Q}}$. Whenever we state a result involving the binomial point process $\mathcal{X}_{n}$, we implicitly assume that $\mathbb{Q}$, and hence $\widehat{\mathbb{Q}}$, are probability measures.

Score functions. Next, we consider the score functions $\left(\xi_{s}\right)_{s \geq 1}$ that form the statistics $H_{s}$ and $H_{n}^{\prime}$ defined at (1.1) and (1.2). We assume that the scores $\left(\xi_{s}\right)_{s \geq 1}$ are measurable functions from $\widehat{\mathbb{X}} \times \mathbf{N}$ to $\mathbb{R}$. To formulate the assumptions of our main results in the next subsection, we need to introduce several crucial concepts for scores. 
DEFINITION (Radius of stabilization). For $s \geq 1$ a measurable map $R_{S}: \widehat{\mathbb{X}} \times$ $\mathbf{N} \rightarrow \mathbb{R}$ is called a radius of stabilization for $\xi_{s}$ if for all $\hat{x}:=\left(x, m_{x}\right) \in \widehat{\mathbb{X}}, \mathcal{M} \in \mathbf{N}$ and finite $\widehat{\mathcal{A}} \subset \widehat{\mathbb{X}}$ with $|\widehat{\mathcal{A}}| \leq 7$,

$$
\xi_{s}\left(\hat{x},(\mathcal{M} \cup\{\hat{x}\} \cup \widehat{\mathcal{A}}) \cap \widehat{B}\left(x, R_{s}(\hat{x}, \mathcal{M} \cup\{\hat{x}\})\right)\right)=\xi_{s}(\hat{x}, \mathcal{M} \cup\{\hat{x}\} \cup \widehat{\mathcal{A}}),
$$

where $\widehat{B}(y, r):=B(y, r) \times \mathbb{M}$ for $y \in \mathbb{X}$ and $r>0$.

Loosely speaking, the condition (2.3) says that the value of $\xi_{s}$ at $\hat{x}$ is wholly determined by the points in $\mathcal{M}$ at distance at most $R_{s}(\hat{x}, \mathcal{M} \cup\{\hat{x}\})$ from $\hat{x}$. We illustrate this definition with an example for $\mathbb{X}=[0,1]^{d}$ and $\mathbb{Q}$ the Lebesgue measure restricted to $[0,1]^{d}$ and without marks. Let $\xi_{s}, s \geq 1$, be given by $\xi_{s}(x, \mathcal{M})=s^{1 / d} \min \{\mathrm{d}(x, y): y \in \mathcal{M} \backslash\{x\}\}$ for $x \in \mathbb{X}$ and $\mathcal{M} \in \mathbf{N}$. This means that $\xi_{s}(x, \mathcal{M})$ is $s^{1 / d}$ times the distance from $x$ to its nearest neighbor and that the corresponding sum of scores is $s^{1 / d}$ times the total edge length of the directed nearest neighbor graph (see Section 3.1 for more details on the undirected $k$-nearest neighbors graph, a similar and slightly more complicated model). Now $R_{S}(x, \mathcal{M}):=$ $\min \{\mathrm{d}(x, y): y \in \mathcal{M} \backslash\{x\}\}$ is a radius of stabilization of $\xi_{s}$ since the ball of this radius around $x$ contains at least one other point of $\mathcal{M} \cup \mathcal{A}$, whence $\xi_{s}(x, \mathcal{M} \cup \mathcal{A})$ is completely determined by the points from $(\mathcal{M} \cup \mathcal{A}) \cap B(0, R(x, \mathcal{M}))$.

Returning to the general set-up, given a point $x \in \mathbb{X}$ we denote by $M_{x}$ the corresponding random mark, which is distributed according to $\mathbb{Q}_{\mathbb{M}}$ and is independent of everything else.

DEFINITION (Exponential stabilization). The scores $\left(\xi_{s}\right)_{s \geq 1}$ [resp., $\left.\left(\xi_{n}\right)_{n \in \mathbb{N}}\right]$ are exponentially stabilizing if there are radii of stabilization $\left(R_{S}\right)_{s \geq 1}$ [resp., $\left.\left(R_{n}\right)_{n \in \mathbb{N}}\right]$ and constants $C_{\text {stab }}, c_{\text {stab }}, \alpha_{\text {stab }} \in(0, \infty)$ such that, for $x \in \mathbb{X}$, $r \geq 0$ and $s \geq 1$,

$$
\mathbb{P}\left(R_{s}\left(\left(x, M_{x}\right), \mathcal{P}_{s} \cup\left\{\left(x, M_{x}\right)\right\}\right) \geq r\right) \leq C_{\text {stab }} \exp \left(-c_{\text {stab }}\left(s^{1 / \gamma} r\right)^{\alpha_{\text {stab }}}\right),
$$

respectively, for $x \in \mathbb{X}, r \geq 0$ and $n \geq 9$,

$$
\mathbb{P}\left(R_{n}\left(\left(x, M_{x}\right), \mathcal{X}_{n-8} \cup\left\{\left(x, M_{x}\right)\right\}\right) \geq r\right) \leq C_{\text {stab }} \exp \left(-c_{\text {stab }}\left(n^{1 / \gamma} r\right)^{\alpha_{\text {stab }}}\right),
$$

where $\gamma$ is the constant from (2.1).

For the radius of stabilization $R_{S}$ given above for the scores related to the directed nearest neighbor graph, the event $R_{S}(x, \mathcal{M} \cup\{x\})>r$ for some $r>0$ is equivalent to $(\mathcal{M} \backslash\{x\}) \cap B(x, r)=\varnothing$. For both Poisson and binomial input the probability of this event decays exponentially in $r$ so that the conditions (2.4) and (2.5) are satisfied and the scores are exponentially stabilizing.

For a finite set $\mathcal{A} \subset \mathbb{X}$, we denote by $\left(\mathcal{A}, M_{\mathcal{A}}\right)$ the random set obtained by equipping each point of $\mathcal{A}$ with a random mark distributed according to $\mathbb{Q}_{\mathbb{M}}$ and independent of everything else. 
Definition [Bounded $(4+p)$ th moment]. Let $p \in[0, \infty)$ be given. The scores $\left(\xi_{s}\right)_{s \geq 1}$ or $\left(\xi_{n}\right)_{n \in \mathbb{N}}$ satisfy a $(4+p)$ th moment condition if there is a constant $C_{p} \in(0, \infty)$ such that for all $\mathcal{A} \subset \mathbb{X}$ with $|\mathcal{A}| \leq 7$,

$$
\sup _{s \in[1, \infty)} \sup _{x \in \mathbb{X}} \mathbb{E}\left|\xi_{s}\left(\left(x, M_{x}\right), \mathcal{P}_{s} \cup\left\{\left(x, M_{x}\right)\right\} \cup\left(\mathcal{A}, M_{\mathcal{A}}\right)\right)\right|^{4+p} \leq C_{p}
$$

or

$$
\sup _{n \in \mathbb{N}, n \geq 9} \sup _{x \in \mathbb{X}} \mathbb{E}\left|\xi_{n}\left(\left(x, M_{x}\right), \mathcal{X}_{n-8} \cup\left\{\left(x, M_{x}\right)\right\} \cup\left(\mathcal{A}, M_{\mathcal{A}}\right)\right)\right|^{4+p} \leq C_{p}
$$

Since the scores of the total edge length of the directed nearest neighbor graph are bounded by $s^{1 / d}$ times the radius of stabilization, which decays exponentially, a straight forward computation reveals that they satisfy the $(4+p)$ th moment condition for any $p \in[0, \infty)$.

We introduce a further notion relevant, for example, for functionals whose variances exhibit surface area order scaling. Throughout we assume without further mention that $K$ is a measurable subset of $\mathbb{X}$ such that the map $\mathbb{X} \ni z \mapsto$ $\mathrm{d}(z, K):=\inf _{y \in K} \mathrm{~d}(z, y)$ is measurable. Here, $\mathrm{d}(z, K)$ is the distance between a point $z \in \mathbb{X}$ and $K$. If there is a sequence in $K$ that is dense with respect to d, the measurability assumption is always satisfied. Moreover, we use the abbreviation $\mathrm{d}_{s}(\cdot, \cdot):=s^{1 / \gamma} \mathrm{d}(\cdot, \cdot), s \geq 1$.

DEFINITION (Exponential decay with distance to $K$ ). The scores $\left(\xi_{s}\right)_{s \geq 1}$, respectively, $\left(\xi_{n}\right)_{n \in \mathbb{N}}$, decay exponentially fast with the distance to $K$ if there are constants $C_{K}, c_{K}, \alpha_{K} \in(0, \infty)$ such that for all $\mathcal{A} \subset \mathbb{X}$ with $|\mathcal{A}| \leq 7$,

$$
\begin{gathered}
\mathbb{P}\left(\xi_{s}\left(\left(x, M_{x}\right), \mathcal{P}_{s} \cup\left\{\left(x, M_{x}\right)\right\} \cup\left(\mathcal{A}, M_{\mathcal{A}}\right)\right) \neq 0\right) \\
\leq C_{K} \exp \left(-c_{K} \mathrm{~d}_{s}(x, K)^{\alpha_{K}}\right)
\end{gathered}
$$

for $x \in \mathbb{X}$ and $s \geq 1$ respectively,

$$
\begin{aligned}
& \mathbb{P}\left(\xi_{n}\left(\left(x, M_{x}\right), \mathcal{X}_{n-8} \cup\left\{\left(x, M_{x}\right)\right\} \cup\left(\mathcal{A}, M_{\mathcal{A}}\right)\right) \neq 0\right) \\
& \leq C_{K} \exp \left(-c_{K} \mathrm{~d}_{n}(x, K)^{\alpha_{K}}\right)
\end{aligned}
$$

for $x \in \mathbb{X}$ and $n \geq 9$.

For the previously discussed scores of the total edge length of the directed nearest neighbor graph, the conditions (2.8) and (2.9) are satisfied for the trivial choice $K=\mathbb{X}, C_{K}=1$ and arbitrary $c_{K}, \alpha_{K} \in(0, \infty)$. As a nontrivial example, we consider the points of the underlying point process of the directed nearest neighbor graph that are closer to the boundary of $\mathbb{X}=[0,1]^{d}$ than to their nearest neighbor. For $s \geq 1$, let $\xi_{s}(x, \mathcal{M})$ be one if $\mathrm{d}\left(x, \partial\left([0,1]^{d}\right)\right)<\min \{\mathrm{d}(x, y): y \in \mathcal{M} \backslash\{x\}\}$ and, otherwise, zero so that the corresponding sums $H_{s}$ and $H_{n}^{\prime}$ are the total numbers of points closer to the boundary of $\mathbb{X}=[0,1]^{d}$ than to their nearest neighbor. Here, the exponential decay condition is satisfied with $K=\partial\left([0,1]^{d}\right)$. The 
idea is that if a point $x \in \mathbb{X}$ is far away from $K$, then with high probability there is some other point of the point process in $\mathbb{X}$ that is closer than $K$. Indeed, $\xi_{s}(x, \mathcal{M} \cup\{x\})=1$ is equivalent to $(\mathcal{M} \backslash\{x\}) \cap B(x, \mathrm{~d}(x, K))=\varnothing$, an event whose probability decays exponentially in $\mathrm{d}(x, K)$ for Poisson and binomial input, whence (2.8) and (2.9) are satisfied. The choice of the set $K$ will be crucial for our main results and will be further discussed after Theorem 2.1.

2.2. Statements of the main results. In this subsection, we present our main results, whose proofs are postponed to Section 5. The following general theorem provides rates of normal convergence for $H_{s}$ and $H_{n}^{\prime}$ in terms of the Kolmogorov distance defined at (1.3). This theorem is a consequence of general theorems from [22] and [20] giving Malliavin-Stein bounds for functionals of Poisson and binomial point processes (see Theorems 4.1 and 4.2 below). We define $\alpha:=\min \left\{\alpha_{\text {stab }}, \alpha_{K}\right\}$ and

$$
I_{K, s}:=s \int_{\mathbb{X}} \exp \left(-\frac{\min \left\{c_{\mathrm{stab}}, c_{K}\right\} p \mathrm{~d}_{s}(x, K)^{\alpha}}{36 \cdot 4^{\alpha+1}}\right) \mathbb{Q}(\mathrm{d} x), \quad s \geq 1,
$$

where all the constants and $K$ are as in Section 2.1. Throughout this paper, $N$ always denotes a standard Gaussian random variable.

THEOREM 2.1. (a) Assume that the score functions $\left(\xi_{s}\right)_{s \geq 1}$ are exponentially stabilizing (2.4), satisfy the moment condition (2.6) for some $p \in(0,1]$, and decay exponentially fast with the distance to a set $K \subseteq \mathbb{X}$, as at (2.8). Then there is a constant $\tilde{C} \in(0, \infty)$ only depending on the constants in $(2.1),(2.4),(2.6)$ and (2.8) such that

$$
\begin{aligned}
& d_{K}\left(\frac{H_{s}-\mathbb{E} H_{s}}{\sqrt{\operatorname{Var} H_{s}}}, N\right) \\
& \quad \leq \tilde{C}\left(\frac{\sqrt{I_{K, s}}}{\operatorname{Var} H_{s}}+\frac{I_{K, s}}{\left(\operatorname{Var} H_{s}\right)^{3 / 2}}+\frac{I_{K, s}^{5 / 4}+I_{K, s}^{3 / 2}}{\left(\operatorname{Var} H_{s}\right)^{2}}\right), \quad s \geq 1 .
\end{aligned}
$$

(b) Assume that the score functions $\left(\xi_{n}\right)_{n \in \mathbb{N}}$ are exponentially stabilizing (2.5), satisfy the moment condition (2.7) for some $p \in(0,1]$, and decay exponentially fast with the distance to a set $K \subseteq \mathbb{X}$, as at (2.9). Let $\left(I_{K, n}\right)_{n \in \mathbb{N}}$ be as in (2.10). Then there is a constant $\tilde{C} \in(0, \infty)$ only depending on the constants in $(2.1),(2.5)$, (2.7) and (2.9) such that

$$
\begin{aligned}
& d_{K}\left(\frac{H_{n}^{\prime}-\mathbb{E} H_{n}^{\prime}}{\sqrt{\operatorname{Var} H_{n}^{\prime}}}, N\right) \\
& \quad \leq \tilde{C}\left(\frac{\sqrt{I_{K, n}}}{\operatorname{Var} H_{n}^{\prime}}+\frac{I_{K, n}}{\left(\operatorname{Var} H_{n}^{\prime}\right)^{3 / 2}}+\frac{I_{K, n}+I_{K, n}^{3 / 2}}{\left(\operatorname{Var} H_{n}^{\prime}\right)^{2}}\right), \quad n \geq 9 .
\end{aligned}
$$


To deduce central limit theorems from Theorem 2.1, we will have to show that $\sqrt{I_{K, s}} / \operatorname{Var} H_{s} \rightarrow 0$ as $s \rightarrow \infty$ and $\sqrt{I_{K, n}} / \operatorname{Var} H_{n}^{\prime} \rightarrow 0$ as $n \rightarrow \infty$, respectively, which heavily depends on the choice of $K$. In the sequel we always assume that $I_{K, s}$ is finite. This is only a restriction for the Poisson case, where $\mathbb{Q}(\mathbb{X})=\infty$ is allowed. Then $I_{K, s}<\infty$ and (2.8) imply that the number of points of $\mathcal{P}_{s}$ with nonvanishing scores is finite almost surely. Now let us discuss some prominent choices for $K$. If there is a set $A \in \mathcal{F}$ such that $\mathbb{Q}(A)<\infty, \mathbb{X} \ni z \mapsto \mathrm{d}(z, A)$ is measurable, and the scores of points outside of $A$ vanish almost surely, we make the choice $K=A$, for which (2.8) and (2.9) are obviously satisfied with $C_{K}=1$ and arbitrary $c_{K}, \alpha_{K} \in(0, \infty)$. This approach, often with $K=A=\mathbb{X}$ so that

$$
I_{\mathbb{X}, s}=s \mathbb{Q}(\mathbb{X}), \quad s \geq 1, \quad \text { and } \quad I_{\mathbb{X}, n}=n \mathbb{Q}(\mathbb{X}), \quad n \in \mathbb{N},
$$

works for many functionals whose variances have volume order, for example, the total edge length of the directed nearest neighbor graph considered above. If $\mathbb{X}$ is $\mathbb{R}^{d}$ or a compact convex subset of $\mathbb{R}^{d}$ such as the unit cube, one sometimes has to choose $K$ to be a $(d-1)$-dimensional subset of $\mathbb{R}^{d}$ to ensure that $\sqrt{I_{K, s}} / \operatorname{Var} H_{s}$ and $\sqrt{I_{K, n}} /$ Var $H_{n}^{\prime}$ vanish. This situation arises, for example, when considering statistics of convex hulls of random samples, Voronoi set approximation, and the total number of maximal points. Problems with surface order scaling of the variance are typically of this form.

Assuming growth bounds on $I_{K, s} / \operatorname{Var} H_{s}$ and $I_{K, n} / \operatorname{Var} H_{n}^{\prime}$, the rates (2.11) and (2.12) nicely simplify into presumably optimal rates (see Section 1 for a discussion of optimality), ready for off-the-shelf use in applications.

COROLlary 2.2. (a) Let the conditions of Theorem 2.1(a) prevail. Assume further that there is a $C \in(0, \infty)$ such that $\sup _{s \geq 1} I_{K, s} / \operatorname{Var} H_{s} \leq C$. Then there is a $\tilde{C}^{\prime} \in(0, \infty)$ only depending on $C$ and the constants in (2.1), (2.4), (2.6) and (2.8) such that

$$
d_{K}\left(\frac{H_{s}-\mathbb{E} H_{s}}{\sqrt{\operatorname{Var} H_{s}}}, N\right) \leq \frac{\tilde{C}^{\prime}}{\sqrt{\operatorname{Var} H_{s}}}, \quad s \geq 1 .
$$

(b) Let the conditions of Theorem 2.1(b) prevail. If there is a $C \in(0, \infty)$ such that $\sup _{n \geq 1} I_{K, n} / \operatorname{Var} H_{n}^{\prime} \leq C$, then there is a $\tilde{C}^{\prime} \in(0, \infty)$ only depending on $C$ and the constants in (2.1), (2.5), (2.7) and (2.9) such that

$$
d_{K}\left(\frac{H_{n}^{\prime}-\mathbb{E} H_{n}^{\prime}}{\sqrt{\operatorname{Var} H_{n}^{\prime}}}, N\right) \leq \frac{\tilde{C}^{\prime}}{\sqrt{\operatorname{Var} H_{n}^{\prime}}}, \quad n \geq 9 .
$$

This corollary is applied in the context of the convex hull of a random sample of points in a smooth convex set in Section 3.4. In this case, the variance is of order $s^{\frac{d-1}{d+1}}\left(n^{\frac{d-1}{d+1}}\right.$ in the binomial setting), and we obtain rates of normal convergence of order $\left(\operatorname{Var} H_{s}\right)^{-1 / 2}=\Theta\left(s^{-(d-1) /(2(d+1))}\right)$ [resp., $\left(\operatorname{Var} H_{n}^{\prime}\right)^{-1 / 2}=$ $\left.\Theta\left(n^{-(d-1) /(2(d+1))}\right)\right]$, which improves upon rates obtained via other methods. 
In the setting $\mathbb{X} \subseteq \mathbb{R}^{d}$, our results admit further simplification, which goes as follows. For $K \subseteq \mathbb{X} \subseteq \mathbb{R}^{d}$ and $r \in(0, \infty)$, let $K_{r}:=\left\{y \in \mathbb{R}^{d}: \mathrm{d}(y, K) \leq r\right\}$ denote the $r$-parallel set of $K$. Recall that the $(d-1)$-dimensional upper Minkowski content of $K$ is given by

$$
\overline{\mathcal{M}}^{d-1}(K):=\limsup _{r \rightarrow 0} \frac{\operatorname{Vol}_{d}\left(K_{r}\right)}{2 r} .
$$

If $K$ is a closed $(d-1)$-rectifiable set in $\mathbb{R}^{d}$ (i.e., the Lipschitz image of a bounded set in $\left.\mathbb{R}^{d-1}\right)$, then $\overline{\mathcal{M}}^{d-1}(K)$ exists and coincides with a scalar multiple of $\mathcal{H}^{d-1}(K)$, the $(d-1)$-dimensional Hausdorff measure of $K$. Given an unbounded set $I \subset(0, \infty)$ and two families of real numbers $\left(a_{i}\right)_{i \in I},\left(b_{i}\right)_{i \in I}$, we use the Landau notation $a_{i}=O\left(b_{i}\right)$ to indicate that $\lim \sup _{i \in I, i \rightarrow \infty}\left|a_{i}\right| /\left|b_{i}\right|<\infty$. If $b_{i}=O\left(a_{i}\right)$, we write $a_{i}=\Omega\left(b_{i}\right)$, whereas if $a_{i}=O\left(b_{i}\right)$ and $b_{i}=O\left(a_{i}\right)$ we write $a_{i}=\Theta\left(b_{i}\right)$.

THEOREM 2.3. Let $\mathbb{X} \subseteq \mathbb{R}^{d}$ be full-dimensional, let $\mathbb{Q}$ have a bounded density with respect to Lebesgue measure and let the conditions of Theorem 2.1 prevail with $\gamma:=d$.

(a) Let $K$ be a full-dimensional compact subset of $\mathbb{X}$ with $\overline{\mathcal{M}}^{d-1}(\partial K)<\infty$. If $\operatorname{Var} H_{s}=\Omega(s)$, respectively, $\operatorname{Var} H_{n}^{\prime}=\Omega(n)$, then there is a constant $c \in(0, \infty)$ such that

$$
d_{K}\left(\frac{H_{s}-\mathbb{E} H_{s}}{\sqrt{\operatorname{Var} H_{s}}}, N\right) \leq \frac{c}{\sqrt{s}}, \quad \text { resp. } \quad d_{K}\left(\frac{H_{n}^{\prime}-\mathbb{E} H_{n}^{\prime}}{\sqrt{\operatorname{Var} H_{n}^{\prime}}}, N\right) \leq \frac{c}{\sqrt{n}}
$$

for $s \geq 1$, respectively, $n \geq 9$.

(b) Let $K$ be a $(d-1)$-dimensional compact subset of $\mathbb{X}$ with $\overline{\mathcal{M}}^{d-1}(K)<$ $\infty$. If $\operatorname{Var} H_{s}=\Omega\left(s^{(d-1) / d}\right)$, respectively, $\operatorname{Var} H_{n}^{\prime}=\Omega\left(n^{(d-1) / d}\right)$, then there is a constant $c \in(0, \infty)$ such that

$$
d_{K}\left(\frac{H_{s}-\mathbb{E} H_{s}}{\sqrt{\operatorname{Var} H_{s}}}, N\right) \leq \frac{c}{s^{\frac{1}{2}-\frac{1}{2 d}}}, \quad \text { resp. } \quad d_{K}\left(\frac{H_{n}^{\prime}-\mathbb{E} H_{n}^{\prime}}{\sqrt{\operatorname{Var} H_{n}^{\prime}}}, N\right) \leq \frac{c}{n^{\frac{1}{2}-\frac{1}{2 d}}}
$$

for $s \geq 1$, respectively, $n \geq 9$.

The constants $c \in(0, \infty)$ in (a) and (b) depend on the constants in (2.1) and (2.4)(2.9), the set $K$ and the behavior of the variances $\operatorname{Var} H_{s}$ and $\operatorname{Var} H_{n}^{\prime}$.

REMARKS. (i) Comparing (2.17) with existing results. The results at (2.17) are applicable in the setting of volume order scaling of the variances, that is, when the variances of $H_{s}$ and $H_{n}^{\prime}$ exhibit scaling proportional to $s$ and $n$. The rate for Poisson input in (2.17) improves upon the rates given by Theorem 2.1 of [30] (see also Lemma 4.4 of [25]), Corollary 3.1 of [5] and Theorem 2.3 in [27], which 
all contain extraneous logarithmic factors and which rely on dependency graph methods. The rate in (2.17) for binomial input is new, as up to now there are no explicit general rates of normal convergence for sums of stabilizing score functions $\xi_{n}$ of binomial input.

(ii) Comparing (2.18) with existing results. The rates at (2.18) are relevant for statistics with surface area rescaling of the variances, that is, when the variance of $H_{s}$ (resp., $H_{n}^{\prime}$ ) exhibits scaling proportional to $s^{1-1 / d}$ (resp., $n^{1-1 / d}$ ). These rates both improve and extend upon the rates given in the main result (Theorem 1.3) in [48]. First, in the case of Poisson input, the rates remove the logarithmic factors present in Theorem 1.3 of [48]. Second, we obtain rates of normal convergence for binomial input, whereas [48] does not treat this situation.

(iii) Unspecified constants. The bounds for normal approximation all involve unspecified constants $\tilde{C}$, $\tilde{C}^{\prime}$ and $c$. By following our proofs, one could explicitly determine these constants in terms of the constants in (2.1), (2.4)-(2.9) (as well as in terms of $C$ in case of Corollary 2.2 and in terms of $K$ and the behavior of Var $H_{S}$ and $\operatorname{Var} H_{n}^{\prime}$ in case of Theorem 2.3). For the sake of readability, we have decided not to take this approach.

(iv) Normal approximation via re-scaling. Let $\mathcal{P}$ be a homogenous Poisson point process of intensity one on $\mathbb{R}^{d}$. Applications often involve showing normal approximation for $\sum_{x \in \mathcal{P} \cap W_{s}} \xi\left(x, \mathcal{P} \cap W_{s}\right)$, where $\xi$ is a stabilizing score function and $W_{s}:=\left[-\frac{1}{2} s^{1 / d}, \frac{1}{2} s^{1 / d}\right]^{d}$. To see that our main results treat this situation, it suffices to put $\mathbb{X}$ to be $W_{1}, \mathbb{Q}$ Lebesgue measure on $K:=W_{1}, \mathcal{P}_{s}$ a Poisson point process of intensity $s \mathbb{Q}$, and $\xi_{s}(x, \mathcal{M}):=\xi\left(s^{1 / d} x, s^{1 / d} \mathcal{M}\right)$. One may likewise deduce central limit theorems for $\sum_{x \in \mathcal{P} \cap W_{s}} \xi(x, \mathcal{P})$ by taking $\mathbb{X}$ to be $\mathbb{R}^{d}, \mathbb{Q}$ Lebesgue measure on $\mathbb{R}^{d}, \mathcal{P}_{s}$ a Poisson point process of intensity $s \mathbb{Q}, K:=W_{1}$ and $\xi_{s}(x, \mathcal{M}):=\xi\left(s^{1 / d} x, s^{1 / d} \mathcal{M}\right)$ when $x \in W_{1}$ and zero otherwise. In this situation, we have $I_{K, x}=\Theta(s)$.

(v) Extensions to random measures. Up to a constant factor, the rates of normal convergence in Theorem 2.1, Corollary 2.2 and Theorem 2.3 hold for the nonlinear statistics $H_{s}(f)=\sum_{x \in \mathcal{P}_{s}} f(x) \xi_{s}\left(x, \mathcal{P}_{s}\right)$ and $H_{n}^{\prime}(f)=\sum_{x \in \mathcal{X}_{n}} f(x) \xi_{n}\left(x, \mathcal{X}_{n}\right)$, obtained by integrating the random measures $\sum_{x \in \mathcal{P}_{s}} \xi_{s}\left(x, \mathcal{P}_{s}\right) \delta_{x}$ and $\sum_{x \in \mathcal{X}_{n}} \xi_{n}\left(x, \mathcal{X}_{n}\right) \delta_{x}$ with a bounded measurable test function $f$ on $\mathbb{X}$. For example, if the assumptions of Theorem 2.3(a) are satisfied with $K=\mathbb{X}, \operatorname{Var}\left(H_{S}(f)\right)=$ $\Omega(s)$, and $\operatorname{Var}\left(H_{n}^{\prime}(f)\right)=\Omega(n)$, then there is a constant $c \in(0, \infty)$ such that

$$
d_{K}\left(\frac{H_{s}(f)-\mathbb{E} H_{s}(f)}{\sqrt{\operatorname{Var} H_{S}(f)}}, N\right) \leq \frac{c}{\sqrt{s}}, \quad s \geq 1,
$$

and

$$
d_{K}\left(\frac{H_{n}^{\prime}(f)-\mathbb{E} H_{n}^{\prime}(f)}{\sqrt{\operatorname{Var} H_{n}^{\prime}(f)}}, N\right) \leq \frac{c}{\sqrt{n}}, \quad n \geq 9 .
$$

Here, the constant $c \in(0, \infty)$ depends on the constants in (2.1) and (2.4)-(2.7), the set $K$ and the behavior of the variances $\operatorname{Var}\left(H_{s}(f)\right)$ and $\operatorname{Var}\left(H_{n}^{\prime}(f)\right)$. The rate 
(2.19) improves upon the main result (Theorem 2.1) of [30] whereas the rate (2.20) is new.

(vi) Extensions to the Wasserstein distance. All quantitative bounds presented in this section also hold for the Wasserstein distance (see also the discussion at the end of Section 4). The Wasserstein distance between random variables $Y$ and $Z$ with $\mathbb{E}|Y|, \mathbb{E}|Z|<\infty$ is given by

$$
d_{W}(Y, Z):=\sup _{h \in \operatorname{Lip}(1)}|\mathbb{E} h(Y)-\mathbb{E} h(Z)|,
$$

where $\operatorname{Lip}(1)$ stands for the set of all functions $h: \mathbb{R} \rightarrow \mathbb{R}$ whose Lipschitz constant is at most one. Since we believe that the Kolmogorov distance $d_{K}$ is more prominent than the Wasserstein distance, the applications in Section 3 are formulated only for $d_{K}$.

(vii) Subsets without influence. Assume that there is a measurable set $\tilde{\mathbb{X}} \subset \mathbb{X}$ such that the scores satisfy

$$
\xi_{s}(x, \mathcal{M})=\mathbf{1}_{\{x \in \tilde{\mathbb{X}}\}} \xi_{s}(x, \mathcal{M} \cap \tilde{\mathbb{X}}), \quad \mathcal{M} \in \mathbf{N}, x \in \mathcal{M}, s \geq 1,
$$

where $\mathcal{M} \cap \tilde{\mathbb{X}}$ stands for the restriction of the point configuration $\mathcal{M}$ to $\tilde{\mathbb{X}}$. In other words, the sum of scores $\sum_{x \in \mathcal{M}} \xi_{s}(x, \mathcal{M})$ only depends on the points of $\mathcal{M}$ which belong to $\tilde{\mathbb{X}}$. In this case, our previous results are still valid if the assumptions (2.1)-(2.9) hold for all $x \in \tilde{\mathbb{X}}$.

(viii) Null sets. In our assumptions (2.1)-(2.9) we require, for simplicity, that some inequalities are satisfied for all $x \in \mathbb{X}$. In case that these only hold for $\mathbb{Q}$-a.e. $x \in \mathbb{X}$, our results are still true. This also applies to comment (vii).

(ix) Extensions to polynomial stabilization. The situation where the radius of stabilization decays polynomially instead of exponentially is known as polynomial stabilization (see [25]). We expect that our results can be proved by following the same route if the exponential terms in (2.4) and (2.8) [resp., (2.5) and (2.9)] are replaced by

$$
\left(s^{1 / \gamma} r\right)^{-\tau_{\text {stab }}} \quad \text { and } \quad \mathrm{d}_{s}(x, K)^{-\tau_{K}} \quad\left(\text { resp. } \quad\left(n^{1 / \gamma} r\right)^{-\tau_{\text {stab }}} \text { and } \mathrm{d}_{n}(x, K)^{-\tau_{K}}\right)
$$

with $\tau_{\text {stab }}, \tau_{K}>0$ sufficiently large. We did not do so as it would have made our proofs more technical and it seems that most examples of interest are exponentially stabilizing.

3. Applications. By appropriately choosing the measure space $(\mathbb{X}, \mathcal{F}, \mathbb{Q})$, the scores $\left(\xi_{s}\right)_{s \geq 1}$ and $\left(\xi_{n}\right)_{n \in \mathbb{N}}$, and the set $K \subset \mathbb{X}$, we may use the general results of Theorem 2.1, Corollary 2.2 and Theorem 2.3 to deduce presumably optimal rates of normal convergence for statistics in geometric probability. For example, in the setting $\mathbb{X}=\mathbb{R}^{d}$, we expect that all of the statistics $H_{s}$ and $H_{n}^{\prime}$ described in $[7,25,28-30]$ consist of sums of scores $\xi_{s}$ and $\xi_{n}$ satisfying the conditions of Theorem 2.3, showing that the statistics in these papers enjoy rates of normal 
convergence (in the Kolmogorov distance) given by the reciprocal of the standard deviation of $H_{s}$ and $H_{n}^{\prime}$, respectively. Previously, the rates in these papers either contained extraneous logarithmic factors, as in the case of Poisson input, or the rates were sometimes nonexistent, as in the case of binomial input. In the following, we do this in detail for some prominent statistics featuring in the stochastic geometry literature, including the $k$-face and intrinsic volume functionals of convex hulls of random samples. Our selection of statistics is illustrative rather than exhaustive and is intended to demonstrate the wide applicability of Theorem 2.1 and the relative simplicity of Corollary 2.2 and Theorem 2.3. In some instances, the rates of convergence are subject to variance lower bounds, a separate problem not addressed here.

We believe that one could use our approach to also deduce presumably optimal rates of normal convergence for statistics of random sequential packing problems as in [41], set approximation via Delaunay triangulations as in [18], generalized spacings as in [6] and general proximity graphs as in [15].

\subsection{Nearest neighbors graphs and statistics of high-dimensional data sets.}

(a) Total edge length of nearest neighbors graphs. Let $(\mathbb{X}, \mathcal{F}, \mathbb{Q})$ be equipped with a semi-metric $\mathrm{d}$ such that (2.1) is satisfied for some $\gamma$ and $\kappa$. We equip $\mathbb{X}$ with a fixed linear order, which is possible by the well-ordering principle. Given $\mathcal{X} \in \mathbf{N}$, $k \in \mathbb{N}$, and $x \in \mathcal{X}$, let $V_{k}(x, \mathcal{X})$ be the set of $k$ nearest neighbors of $x$, that is, the $k$ closest points of $x$ in $\mathcal{X} \backslash\{x\}$. In case that that these $k$ points are not unique, we break the tie via the fixed linear order on $\mathbb{X}$. The (undirected) nearest neighbor graph $\mathrm{NG}_{1}(\mathcal{X})$ is the graph with vertex set $\mathcal{X}$ obtained by including an edge $\{x, y\}$ if $y \in V_{1}(x, \mathcal{X})$ and/or $x \in V_{1}(y, \mathcal{X})$. More generally, the (undirected) $k$-nearest neighbors graph $\mathrm{NG}_{k}(\mathcal{X})$ is the graph with vertex set $\mathcal{X}$ obtained by including an edge $\{x, y\}$ if $y \in V_{k}(x, \mathcal{X})$ and/or $x \in V_{k}(y, \mathcal{X})$. For all $q \geq 0$, define

$$
\xi^{(q)}(x, \mathcal{X}):=\sum_{y \in V_{k}(x, \mathcal{X})} \rho^{(q)}(x, y),
$$

where $\rho^{(q)}(x, y):=\mathrm{d}(x, y)^{q} / 2$ if $x$ and $y$ are mutual $k$-nearest neighbors, that is, $x \in V_{k}(y, \mathcal{X})$ and $y \in V_{k}(x, \mathcal{X})$, and otherwise $\rho^{(q)}(x, y):=\mathrm{d}(x, y)^{q}$. The total edge length of the undirected $k$-nearest neighbors graph on $\mathcal{X}$ with $q$ th power weighted edges is

$$
L_{\mathrm{NG}_{k}}^{(q)}(\mathcal{X})=\sum_{x \in \mathcal{X}} \xi^{(q)}(x, \mathcal{X}) .
$$

As usual $\mathcal{P}_{s}$ is a Poisson point process on $\mathbb{X}$ with intensity measure $s \mathbb{Q}$ and $\mathcal{X}_{n}$ is a binomial point process of $n$ points in $\mathbb{X}$ distributed according to $\mathbb{Q}$. We assume in the following that $(\mathbb{X}, \mathcal{F}, \mathbb{Q})$ satisfies, beside $(2.1)$,

$$
\inf _{x \in \mathbb{X}} \mathbb{Q}(B(x, r)) \geq c r^{\gamma}, \quad r \in[0, \operatorname{diam}(\mathbb{X})],
$$

where $\gamma$ is the constant from (2.1), diam $(\mathbb{X})$ stands for the diameter of $\mathbb{X}$ and $c>0$. 
THEOREM 3.1. If $q \geq 0$ and $\operatorname{Var}\left(L_{\mathrm{NG}_{k}}^{(q)}\left(\mathcal{P}_{s}\right)\right)=\Omega\left(s^{1-2 q / \gamma}\right)$, then there is $a$ $\tilde{C} \in(0, \infty)$ such that

$$
d_{K}\left(\frac{L_{\mathrm{NG}_{k}}^{(q)}\left(\mathcal{P}_{s}\right)-\mathbb{E} L_{\mathrm{NG}_{k}}^{(q)}\left(\mathcal{P}_{s}\right)}{\sqrt{\operatorname{Var} L_{\mathrm{NG}_{k}}^{(q)}\left(\mathcal{P}_{s}\right)}}, N\right) \leq \frac{\tilde{C}}{\sqrt{s}}, \quad s \geq 1
$$

whereas if $\operatorname{Var}\left(L_{\mathrm{NG}_{k}}^{(q)}\left(\mathcal{X}_{n}\right)\right)=\Omega\left(n^{1-2 q / \gamma}\right)$, then

$$
d_{K}\left(\frac{L_{\mathrm{NG}_{k}}^{(q)}\left(\mathcal{X}_{n}\right)-\mathbb{E} L_{\mathrm{NG}_{k}}^{(q)}\left(\mathcal{X}_{n}\right)}{\sqrt{\operatorname{Var} L_{\mathrm{NG}_{k}}^{(q)}\left(\mathcal{X}_{n}\right)}}, N\right) \leq \frac{\tilde{C}}{\sqrt{n}}, \quad n \geq 9 .
$$

REMARKS. (i) Comparison with previous work. Research has focused on central limit theorems for $L_{\mathrm{NG}_{k}}^{(q)}\left(\mathcal{P}_{s}\right), s \rightarrow \infty$, and $L_{\mathrm{NG}_{k}}^{(q)}\left(\mathcal{X}_{n}\right), n \rightarrow \infty$, when $\mathbb{X}$ is a full-dimensional subset of $\mathbb{R}^{d}$ and where $\mathrm{d}$ is the usual Euclidean distance. This includes the seminal work [8], the paper [1] and the more recent works [27, 28, 30]. When $\mathbb{X}$ is a submanifold of $\mathbb{R}^{d}$ equipped with the Euclidean metric on $\mathbb{R}^{d}$, the paper [31] develops the limit theory for $L_{\mathrm{NG}_{k}}^{(q)}\left(\mathcal{P}_{s}\right), s \rightarrow \infty$, and $L_{\mathrm{NG}_{k}}^{(q)}\left(\mathcal{X}_{n}\right), n \rightarrow \infty$. When $\mathbb{X}$ is a compact convex subset of $\mathbb{R}^{d}$, the paper [22] establishes the presumably optimal $O\left(s^{-1 / 2}\right)$ rate of normal convergence for $L_{\mathrm{NG}_{k}}^{(q)}\left(\mathcal{P}_{s}\right)$.

The rate for binomial input (3.4) improves upon the rate of convergence in the Wasserstein distance $d_{W}$ given by

$$
d_{W}\left(\frac{L_{\mathrm{NG}_{k}}^{(q)}\left(\mathcal{X}_{n}\right)-\mathbb{E} L_{\mathrm{NG}_{k}}^{(q)}\left(\mathcal{X}_{n}\right)}{\sqrt{\operatorname{Var} L_{\mathrm{NG}_{k}}^{(q)}\left(\mathcal{X}_{n}\right)}}, N\right)=O\left(\frac{k^{4} \tilde{\gamma}_{p}^{2 / p}}{n^{(p-8) / 2 p}}+\frac{k^{3} \tilde{\gamma}_{p}^{3 / p}}{n^{(p-6) / 2 p}}\right),
$$

as in Theorem 3.4 of [10] as well as the same rate in the Kolmogorov distance as in Section 6.3 of [20]. Here, $\tilde{\gamma}_{p}:=\mathbb{E}\left|n^{q / \gamma} \xi^{(q)}\left(X_{1}, \mathcal{X}_{n}\right)\right|^{p}$ and $p>8$. For all $\varepsilon>0$, we have $\mathbb{P}\left(n^{q / \gamma} \xi^{(q)}\left(X_{1}, \mathcal{X}_{n}\right)>\varepsilon\right)=\left(1-C \varepsilon^{\gamma} / n\right)^{n}$ and it follows that $\tilde{\gamma}_{p}^{1 / p} \uparrow \infty$ as $p \rightarrow \infty$. Thus, by letting $p \rightarrow \infty$, we do not recover the $O\left(n^{-1 / 2}\right)$ rate in (3.5), but only achieve the rate $O\left(n^{-1 / 2}(\log n)^{\tau}\right)$ with some $\tau>0$.

However, the discussed papers neither provide the presumably optimal $O\left(n^{-1 / 2}\right)$ rate of normal convergence for $L_{\mathrm{NG}_{k}}^{(q)}\left(\mathcal{X}_{n}\right)$ in the $d_{K}$ distance, nor do they consider input on arbitrary metric spaces. Theorem 3.1 rectifies this.

(ii) Variance bounds. When $\mathbb{X}$ is a full-dimensional compact convex subset of $\mathbb{R}^{d}$, then $\gamma=d, \operatorname{Var}\left(L_{\mathrm{NG}_{k}}^{(q)}\left(\mathcal{P}_{s}\right)\right)=\Theta\left(s^{1-2 q / \gamma}\right)$, and $\operatorname{Var}\left(L_{\mathrm{NG}_{k}}\left(\mathcal{X}_{n}\right)\right)=$ $\Theta\left(n^{1-2 q / \gamma}\right)$, which follows from Theorem 2.1 and Lemma 6.3 of [28] [these results treat the case $q=1$ but the proofs easily extend to arbitrary $q \in(0, \infty)]$. Thus we obtain the required variance lower bounds of Theorem 3.1. If $\operatorname{Var}\left(L_{\mathrm{NG}_{k}}^{(q)}\left(\mathcal{P}_{s}\right)\right)=$ $\Omega\left(s^{1-2 q / \gamma}\right)$ does not hold, then the convergence rate in (3.3) is replaced by (2.11) 
with $I_{K, s}$ set to $s$, with a similar statement if $\operatorname{Var}\left(L_{\mathrm{NG}_{k}}\left(\mathcal{X}_{n}\right)\right)=\Omega\left(n^{1-2 q / \gamma}\right)$ does not hold.

(iii) Extension of Theorem 3.1. The directed $k$-nearest neighbors graph, denoted $\mathrm{NG}_{k}^{\prime}(\mathcal{X})$, is the directed graph with vertex set $\mathcal{X}$ obtained by including a directed edge from each point to each of its $k$ nearest neighbors. The total edge length of the directed $k$-nearest neighbors graph on $\mathcal{X}$ with $q$ th power-weighted edges is

$$
L_{\mathrm{NG}_{k}^{\prime}}^{(q)}(\mathcal{X})=\sum_{x \in \mathcal{X}} \tilde{\xi}^{(q)}(x, \mathcal{X}),
$$

where

$$
\tilde{\xi}^{(q)}(x, \mathcal{X}):=\sum_{y \in V_{k}(x, \mathcal{X})} \mathrm{d}(x, y)^{q} .
$$

The proof of Theorem 3.1 given below shows that the analogs of (3.3) and (3.4) hold for $L_{\mathrm{NG}_{k}^{\prime}}^{(q)}\left(\mathcal{P}_{s}\right)$ and $L_{\mathrm{NG}_{k}^{\prime}}^{(q)}\left(\mathcal{X}_{n}\right)$ as well. See also the running example in Section 2.1 for the case $q=k=1$.

Proof of TheOREM 3.1. In the following, we prove (3.3). We deduce this from Corollary 2.2 with $\xi_{s}\left(x, \mathcal{P}_{s}\right)$ set to $s^{q / \gamma} \xi^{(q)}\left(x, \mathcal{P}_{s}\right)$, with $\xi^{(q)}$ as at (3.1) and with $K$ set to $\mathbb{X}$. Recalling the terminology of Corollary 2.2, we have $H_{s}:=$ $s^{q / \gamma} L_{\mathrm{NG}_{k}}^{(q)}\left(\mathcal{P}_{s}\right)=\sum_{x \in \mathcal{P}_{s}} \xi_{s}\left(x, \mathcal{P}_{s}\right)$, with $\operatorname{Var} H_{s}=\operatorname{Var}\left(s^{q / \gamma} \sum_{x \in \mathcal{P}_{s}} \xi^{(q)}\left(x, \mathcal{P}_{s}\right)\right)=$ $\Omega(s)$, by assumption. Recall from (2.13) that $I_{K, s}=\Theta(s)$. We claim that $R_{S}(x, \mathcal{X} \cup\{x\}):=3 \mathrm{~d}\left(x, x_{k N N}(x, \mathcal{X} \cup\{x\})\right)$ is a radius of stabilization for $\xi_{s}(x, \mathcal{X} \cup\{x\})$, where $x_{k N N}(x, \mathcal{X} \cup\{x\})$ is the point of $V_{k}(x, \mathcal{X} \cup\{x\})$ with the maximal distance to $x$. Indeed, if a point $y$ is a $k$-nearest neighbor of $x$, then all of its $k$-nearest neighbors must belong to $B\left(y, 2 \mathrm{~d}\left(x, x_{k N N}(x, \mathcal{X} \cup\{x\})\right)\right)$, since this ball contains with $x$ and its $k-1$ nearest neighbors enough potential $k$-nearest neighbors for $y$. Since $R_{S}(x, \mathcal{X} \cup\{x\})$ is decreasing in $\mathcal{X}$, we do not need to add a deterministic point set $\mathcal{A}$.

We now show that $R_{S}\left(x, \mathcal{P}_{S} \cup\{x\}\right)$ satisfies exponential stabilization (2.4). Notice that

$$
\mathbb{P}\left(R_{s}\left(x, \mathcal{P}_{s} \cup\{x\}\right)>r\right)=\mathbb{P}\left(\mathcal{P}_{s}(B(x, r / 3)<k)\right), \quad r \geq 0 .
$$

The number of points from $\mathcal{P}_{s}$ in $B(x, r / 3)$ follows a Poisson distribution with parameter $s \mathbb{Q}(B(x, r / 3))$. By (3.2), this exceeds $c s(r / 3)^{\gamma}$ if $r \in[0,3 \operatorname{diam}(\mathbb{X})]$. By a Chernoff bound for the Poisson distribution (e.g., Lemma 1.2 of [24]), there is another constant $\tilde{c} \in(0, \infty)$ such that

$$
\mathbb{P}\left(R_{s}\left(x, \mathcal{P}_{s} \cup\{x\}\right)>r\right) \leq k \exp \left(-\tilde{c} s r^{\gamma}\right), \quad r \in[0,3 \operatorname{diam}(\mathbb{X})] .
$$

This also holds for $r>3 \operatorname{diam}(\mathbb{X})$, since $\mathbb{P}\left(R_{S}\left(x, \mathcal{P}_{s} \cup\{x\}\right) \geq r\right)=0$ in this case. This gives (2.4), with $\alpha_{\text {stab }}=\gamma, c_{\text {stab }}=\tilde{c}$, and $C_{\text {stab }}=k$. We may modify this argument to obtain exponential stabilization with respect to binomial input as at (2.5). 
For all $q \in[0, \infty)$, the scores $\left(\xi_{s}\right)_{s \geq 1}$ also satisfy the $(4+p)$ th moment condition (2.6) for all $p \in[0, \infty)$ since

$$
\xi_{s}\left(x, \mathcal{P}_{s} \cup\{x\} \cup \mathcal{A}\right) \leq k s^{q / \gamma} \mathrm{d}\left(x, x_{k N N}\left(x, \mathcal{P}_{s} \cup\{x\}\right)\right)^{q}
$$

for all $\mathcal{A} \subset \mathbb{X}$ with $|\mathcal{A}| \leq 7$, and the above computation shows that $s^{q / \gamma} \mathrm{d}(x$, $\left.x_{k N N}\left(x, \mathcal{P}_{s} \cup\{x\}\right)\right)^{q}$ has an exponentially decaying tail. The bound (3.3) follows by Corollary 2.2. The proof of (3.4) is similar. This completes the proof of Theorem 3.1.

(b) Statistics of high-dimensional data sets. In the case that $\mathbb{X}$ is an $m$ dimensional $C^{1}$-submanifold of $\mathbb{R}^{d}$, with $\mathrm{d}$ the Euclidean distance in $\mathbb{R}^{d}$, the $d i$ rected nearest neighbors graph version of Theorem 3.1 [cf. Remark (iii) above] may be refined to give rates of normal convergence for statistics of highdimensional nonlinear data sets. This goes as follows. Recall that high-dimensional nonlinear data sets are typically modeled as the realization of $\mathcal{X}_{n}:=\left\{X_{1}, \ldots, X_{n}\right\}$, with $X_{i}, 1 \leq i \leq n$, i.i.d. copies of a random variable $X$ having support on an unknown (nonlinear) manifold $\mathbb{X}$ embedded in $\mathbb{R}^{d}$. Typically the coordinate representation of $X_{i}$ is unknown, but the interpoint distances are known. Given this information, the goal is to establish estimators of global characteristics of $\mathbb{X}$, including intrinsic dimension, as well as global properties of the distribution of $X$, such as Rényi entropy. Recall that if the distribution of the random variable $X$ has a Radon-Nikodym derivative $f_{X}$ with respect to the uniform measure on $\mathbb{X}$, then given $\rho \in(0, \infty), \rho \neq 1$, the Rényi $\rho$-entropy of $X$ is

$$
H_{\rho}\left(f_{X}\right):=(1-\rho)^{-1} \log \int_{\mathbb{X}} f_{X}(x)^{\rho} \mathrm{d} x .
$$

Let $\mathbb{X}$ be an $m$-dimensional subset of $\mathbb{R}^{d}, m \leq d$, equipped with the Euclidean metric d on $\mathbb{R}^{d}$. Henceforth, assume $\mathbb{X}$ is an $m$-dimensional $C^{1}$-submanifold-withboundary (see Section 2.1 of [31] for details and precise definitions). Let $\mathbb{Q}$ be a measure on $\mathbb{X}$ with a bounded density $f_{X}$ with respect to the uniform surface measure on $\mathbb{X}$ such that condition (2.1) is satisfied with $\gamma:=m$. Note that Example 2 (Section 2) provides conditions which guarantee that (2.1) holds. Assume $f_{X}$ is bounded away from zero and infinity, and

$$
\inf _{x} \mathbb{Q}(B(x, r)) \geq c r^{m}, \quad r \in[0, \operatorname{diam}(\mathbb{X})],
$$

with some constant $c \in(0, \infty)$. The latter condition is called the "locally conic" condition in [31] (cf. (2.3) in [31]).

Under the above conditions and given Poisson input $\mathcal{P}_{S}$ with intensity $s f_{X}$, the main results of [31] establish rates of normal convergence for estimators of intrinsic dimension, estimators of Rényi entropy, and for Vietoris-Rips clique counts (see Section 2 of [31] for precise statements). However, these rates contain extraneous logarithmic factors and [31] also stops short of establishing rates of normal 
convergence when Poisson input is replaced by binomial input. In what follows, we rectify this for estimators of Rényi entropy. The methods potentially apply to yield rates of normal convergence for estimators of Shannon entropy and intrinsic dimension, but this lies beyond the scope of this paper.

When $f_{X}$ satisfies the assumptions stated above and is also continuous on $\mathbb{X}$, then $n^{q / m-1} L_{\mathrm{NG}_{1}^{\prime}}^{(q)}\left(\mathcal{X}_{n}\right)$ is a consistent estimator of a multiple of $\int_{\mathbb{X}} f_{X}(x)^{1-q / m} \mathrm{~d} x$, as shown in Theorem 2.2 of [31]. The following result establishes a rate of normal convergence for $L_{\mathrm{NG}_{k}^{\prime}}^{(q)}\left(\mathcal{X}_{n}\right)$ and, in particular, for the estimator $n^{q / m-1} L_{\mathrm{NG}_{1}^{\prime}}^{(q)}\left(\mathcal{X}_{n}\right)$.

THEOREM 3.2. If $k \in \mathbb{N}$ and $q \in(0, \infty)$, then there is a constant $c \in(0, \infty)$ such that

$$
d_{K}\left(\frac{L_{\mathrm{NG}_{k}^{\prime}}^{(q)}\left(\mathcal{X}_{n}\right)-\mathbb{E} L_{\mathrm{NG}_{k}^{\prime}}^{(q)}\left(\mathcal{X}_{n}\right)}{\sqrt{\operatorname{Var} L_{\mathrm{NG}_{k}^{\prime}}^{(q)}\left(\mathcal{X}_{n}\right)}}, N\right) \leq \frac{c}{\sqrt{n}}, \quad n \geq 9
$$

A similar result holds if the binomial input $\mathcal{X}_{n}$ is replaced by Poisson input.

REMARKS. (i) We have to exclude the case $q=0$ since $L_{\mathrm{NG}_{1}^{\prime}}^{(0)}\left(\mathcal{X}_{n}\right)=k n$ if $n>k$. For the Poisson case a central limit theorem still holds, but becomes trivial since we have $L_{\mathrm{NG}_{1}^{\prime}}^{(0)}\left(\mathcal{P}_{s}\right)=k\left|\mathcal{P}_{s}\right|$ if $\left|\mathcal{P}_{s}\right| \geq k+1$.

(ii) In the same vein, as described in Remark (i) following Theorem 3.1, Theorem 3.4 of [10] yields a rate of normal convergence for $L_{\mathrm{NG}_{1}^{\prime}}^{(q)}\left(\mathcal{X}_{n}\right)$ in the Wasserstein distance $d_{W}$ given by the right-hand side of (3.5). However, the bound (3.6) is superior and is moreover expressed in the Kolmogorov distance $d_{K}$. When the input $\mathcal{X}_{n}$ is replaced by Poisson input $\mathcal{P}_{s}$, we obtain the rate of normal convergence $O\left(s^{-1 / 2}\right)$, improving upon the rates of [30, 31].

Proof of TheOrem 3.2. Appealing to the method of proof in Theorem 2.3 of [31] and the variance lower bounds of Theorem 6.1 of [28], we see that $\operatorname{Var} L_{\mathrm{NG}_{k}^{\prime}}^{(q)}\left(\mathcal{X}_{n}\right)=\Theta\left(n^{1-2 q / m}\right)$ and $\operatorname{Var} L_{\mathrm{NG}_{k}^{\prime}}^{(q)}\left(\mathcal{P}_{s}\right)=\Theta\left(s^{1-2 q / m}\right)$. The proof follows now the proof of Theorem 3.1.

3.2. Maximal points. Consider the cone $\mathrm{Co}=\left(\mathbb{R}^{+}\right)^{d}$ with apex at the origin of $\mathbb{R}^{d}, d \geq 2$. Given $\mathcal{X} \in \mathbf{N}, x \in \mathcal{X}$ is called maximal if (Co $\left.\oplus x\right) \cap \mathcal{X}=\{x\}$. In other words, a point $x=\left(x_{1}, \ldots, x_{d}\right) \in \mathcal{X}$ is maximal if there is no other point $\left(z_{1}, \ldots, z_{d}\right) \in \mathcal{X}$ with $z_{i} \geq x_{i}$ for all $1 \leq i \leq d$. The maximal layer $m_{\mathrm{Co}}(\mathcal{X})$ is the collection of maximal points in $\mathcal{X}$. Let $M_{\mathrm{Co}}(\mathcal{X}):=\operatorname{card}\left(m_{\mathrm{Co}}(\mathcal{X})\right)$. Maximal 
points are of broad interest in computational geometry and economics; see [11, 32, 44].

Put

$$
\mathbb{X}:=\left\{x \in[0, \infty)^{d}: F(x) \leq 1\right\},
$$

where $F:[0, \infty)^{d} \rightarrow \mathbb{R}^{+}$is a strictly increasing function of each coordinate variable, satisfies $F(0)<1$, is continuously differentiable, and has continuous partials $F_{i}, 1 \leq i \leq d$, bounded away from zero and infinity. Let $\mathbb{Q}$ be a measure on $\mathbb{X}$ with Radon-Nikodym derivative $g$ with respect to Lebesgue measure on $\mathbb{X}$, with $g$ bounded away from zero and infinity. As usual, $\mathcal{P}_{s}$ is the Poisson point process with intensity $s \mathbb{Q}$ and $\mathcal{X}_{n}$ is a binomial point process of $n$ i.i.d. points distributed according to $\mathbb{Q}$.

THEOREM 3.3. There is a constant $c \in(0, \infty)$ such that

$$
d_{K}\left(\frac{M_{\mathrm{Co}}\left(\mathcal{P}_{S}\right)-\mathbb{E} M_{\mathrm{Co}}\left(\mathcal{P}_{S}\right)}{\sqrt{\operatorname{Var} M_{\mathrm{Co}}\left(\mathcal{P}_{S}\right)}}, N\right) \leq c s^{-\frac{1}{2}+\frac{1}{2 d}}, \quad s \geq 1 .
$$

Assuming $\operatorname{Var} M_{\mathrm{Co}}\left(\mathcal{X}_{n}\right)=\Omega\left(n^{(d-1) / d}\right)$, the binomial counterpart to (3.7) holds, with $\mathcal{P}_{s}$ replaced by $\mathcal{X}_{n}$.

REMARKS. (i) Existing results. The rates of normal convergence given by Theorem 3.3 improve upon those given in [4] for Poisson and binomial input for the bounded Wasserstein distance and in [5] and [48] for Poisson input for the Kolmogorov distance. While these findings are also proved via the Stein method, the local dependency methods employed there all incorporate extraneous logarithmic factors. Likewise, when $d=2$, the paper [2] provides rates of normal convergence in the Kolmogorov distance for binomial input, but aside from the special case that $F$ is linear, the rates incorporate extraneous logarithmic factors. The precise approximation bounds of Theorem 3.3 remove the logarithmic factors in $[2,4,5$, 48].

(ii) We have taken $\mathrm{Co}=\left(\mathbb{R}^{+}\right)^{\mathrm{d}}$ to simplify the presentation, but the results extend to general cones which are subsets of $\left(\mathbb{R}^{+}\right)^{d}$ and which have apex at the origin.

Proof of TheOrem 3.3. We deduce this theorem from Theorem 2.3(b) and consider score functions

$$
\zeta(x, \mathcal{X}):= \begin{cases}1 & \text { if }((\operatorname{Co} \oplus x) \cap \mathbb{X}) \cap \mathcal{X}=\{x\}, \\ 0 & \text { otherwise }\end{cases}
$$

Notice that $M_{\mathrm{Co}}(\mathcal{X})=\sum_{x \in \mathcal{P}_{s}} \zeta(x, \mathcal{X})$. Put $K:=\left\{x \in[0, \infty)^{d}: F(x)=1\right\}$. The assumptions on $F$ imply $\overline{\mathcal{M}}^{d-1}(K)<\infty$. In the following, we only prove (3.7) for Poisson input, as the proof for binomial input is similar. Thus we only need 
to show that the scores $\zeta_{s} \equiv \zeta$ satisfy the conditions of Theorem 2.3(b). First, $\zeta$ is bounded and so satisfies the $(4+p)$ th moment condition (2.6) for all $p \in$ $[0, \infty)$. As shown in [48] (see proof of Theorem 2.5 in Section 6), $\zeta_{s}$ also satisfy exponential stabilization (2.4) with $\alpha_{\text {stab }}=d$ with respect to Poisson input $\mathcal{P}_{s}$. Also, we assert that the scores decay exponentially fast with the distance to $K$ with $\alpha_{K}=d$. To see this, let $r(x):=\mathrm{d}(x, K)$ be the distance between $x$ and $K$ and note that $(\operatorname{Co} \oplus x) \cap \mathbb{X}$ contains the set $S(x):=B(x, r(x)) \cap(\operatorname{Co} \oplus x)$. It follows that

$$
\begin{aligned}
\mathbb{P}\left(\zeta\left(x, \mathcal{P}_{s} \cup\{x\}\right) \neq 0\right) & =\mathbb{P}\left(((\operatorname{Co} \oplus x) \cap \mathbb{X}) \cap \mathcal{P}_{s}=\{x\}\right) \\
& =\exp \left(-s \int_{(\operatorname{Co} \oplus x) \cap \mathbb{X}} \mathrm{d} \mathbb{Q}\right) \\
& \leq \exp (-s \mathbb{Q}(S(x))) \\
& \leq \exp \left(-\bar{c} \mathrm{~d}_{s}(x, K)^{d}\right)
\end{aligned}
$$

with some constant $\bar{c}:=\bar{c}(\mathbb{Q}) \in(0, \infty)$, and thus (2.8) holds with $\alpha_{K}=d$.

We now show $\operatorname{Var} M_{\mathrm{Co}}\left(\mathcal{P}_{s}\right)=\Theta\left(s^{(d-1) / d}\right)$. The hypotheses on $F$ imply that there are $M=\Theta\left(s^{(d-1) / d}\right)$ disjoint sets $S_{i}:=\left(\operatorname{Co} \oplus x_{i}\right) \cap \mathbb{X}, i=1, \ldots, M$, with $x_{i} \in \mathbb{X}$, such that $Q_{i}:=\left[0, s^{-1 / d}\right]^{d} \oplus x_{i} \subset S_{i}$ and $x_{i}+s^{-1 / d} e \in K$, where $e=$ $(1, \ldots, 1) \in \mathbb{R}^{d}$. Given $x_{i}$, for $1 \leq j \leq d$ define $d$ subcubes of $Q_{i}$,

$$
Q_{i j}:=\left(\frac{2}{3} s^{-1 / d}, s^{-1 / d}\right]^{j-1} \times\left[0, \frac{1}{3} s^{-1 / d}\right) \times\left(\frac{2}{3} s^{-1 / d}, s^{-1 / d}\right]^{d-j} \oplus x_{i},
$$

as well as the central cube $\tilde{Q}_{i}:=\prod_{j=1}^{d}\left[\frac{1}{3} s^{-1 / d}, \frac{2}{3} s^{-1 / d}\right] \oplus x_{i}$. All cubes thus constructed are disjoint. Say that $S_{i}, 1 \leq i \leq M$, is admissible if there are points

$$
p_{i j} \in \mathcal{P}_{s} \cap Q_{i j}, \quad 1 \leq j \leq d,
$$

which are maximal and $S_{i} \backslash \tilde{Q}_{i}$ contains no other points in $\mathcal{P}_{s}$. Given that $S_{i}$ is admissible, we assert that the maximality status of points in $\mathcal{P}_{s} \cap \tilde{Q}_{i}^{c}$ is unaffected by the (possibly empty) configuration of $\mathcal{P}_{s}$ inside $\tilde{Q}_{i}$. Indeed, if $x \in \mathcal{P}_{s} \cap \tilde{Q}_{i}^{c} \cap Q_{i}$, then $x \in\left\{p_{i j}\right\}_{j=1}^{d}$ and so $(\operatorname{Co} \oplus x) \cap \tilde{Q}_{i}=\varnothing$, showing the assertion in this case. On the other hand, if $x \in \mathcal{P}_{s} \cap \tilde{Q}_{i}^{c} \cap Q_{i}^{c}$ and if $(\operatorname{Co} \oplus x) \cap \tilde{Q}_{i} \neq \varnothing$, then $\operatorname{Co} \oplus x$ must contain at least one of the cubes $Q_{i j}$, thus $\operatorname{Co} \oplus x$ contains at least one of the points $\left\{p_{i j}\right\}_{j=1}^{d}$, and hence $\zeta\left(x, \mathcal{P}_{s}\right)$ vanishes. Let $I$ be the indices $i \in\{1, \ldots, M\}$ such that $S_{i}$ is admissible.

Let $\mathcal{F}_{S}$ be the sigma algebra generated by $I$ and $\mathcal{P}_{S} \cap\left(\mathbb{X} \backslash \bigcup_{i \in I} \tilde{Q}_{i}\right)$, including the maximal points $\left\{\left\{p_{i j}\right\}_{j=1}^{d}\right\}_{i \in I}$. Conditional on $\mathcal{F}_{s}$, note that $\zeta\left(x, \mathcal{P}_{s}\right)$ is deterministic for $x \in \mathcal{P}_{s} \cap\left(\mathbb{X} \backslash \bigcup_{i \in I} \tilde{Q}_{i}\right)$. The conditional variance formula gives

$$
\operatorname{Var} M_{\mathrm{Co}}\left(\mathcal{P}_{s}\right) \geq \mathbb{E} \operatorname{Var}\left[\sum_{x \in \mathcal{P}_{s} \cap \bigcup_{i \in I} \tilde{Q}_{i}} \zeta\left(x, \mathcal{P}_{s}\right)+\sum_{x \in \mathcal{P}_{s} \cap\left(\mathbb{X} \backslash \bigcup_{i \in I} \tilde{Q}_{i}\right)} \zeta\left(x, \mathcal{P}_{s}\right) \mid \mathcal{F}_{s}\right]
$$




$$
\begin{aligned}
& =\mathbb{E} \operatorname{Var}\left[\sum_{i \in I} \sum_{x \in \mathcal{P}_{s} \cap \tilde{Q}_{i}} \zeta\left(x, \mathcal{P}_{s}\right) \mid \mathcal{F}_{s}\right] \\
& =\mathbb{E} \sum_{i \in I} \operatorname{Var}\left[\sum_{x \in \mathcal{P}_{s} \cap \tilde{Q}_{i}} \zeta\left(x, \mathcal{P}_{s}\right) \mid \mathcal{F}_{s}\right],
\end{aligned}
$$

where the last equality follows by independence of $\sum_{x \in \mathcal{P}_{s} \cap \tilde{Q}_{i}} \zeta\left(x, \mathcal{P}_{s}\right), i \in I$. For $i \in I$, the number of maximal points in $\tilde{Q}_{i}$ only depends on the restriction of $\mathcal{P}_{s}$ to $\tilde{Q}_{i}$, and thus exhibits nonzero variability. Together with the bounds on $g$, we obtain that $\operatorname{Var}\left[\sum_{x \in \mathcal{P}_{s} \cap \tilde{Q}_{i}} \zeta\left(x, \mathcal{P}_{s}\right) \mid \mathcal{F}_{s}\right] \geq c_{1}>0$ uniformly in $i \in I$. Since $\mathbb{E} \operatorname{card}(I) \geq c_{2} s^{(d-1) / d}$ with $c_{2} \in(0, \infty)$, the asserted variance lower bound follows. Theorem 2.3(b) gives (3.7).

The proof method in [48] is for Poisson input $\mathcal{P}_{s}$, but it may be easily extended to show that $\left(\zeta_{n}\right)_{n \in \mathbb{N}}$ are exponentially stabilizing with respect to binomial input and that $\left(\zeta_{n}\right)_{n \in \mathbb{N}}$ decay exponentially fast with the distance to $K$. Thus the conditions of Theorem 2.3(b) are satisfied, and so (3.7) follows from (2.14), concluding the proof of Theorem 3.3.

3.3. Set approximation via Voronoi tessellations. Throughout this subsection, let $\mathbb{X}:=[-1 / 2,1 / 2]^{d}, d \geq 2$, and let $A \subset \operatorname{int}(\mathbb{X})$ be a full-dimensional subset of $\mathbb{R}^{d}$. Let $\mathbb{Q}$ be the uniform measure on $\mathbb{X}$. For $\mathcal{X} \in \mathbf{N}$ and $x \in \mathcal{X}$ the Voronoi cell $C(x, \mathcal{X})$ is the set of all $z \in \mathbb{X}$ such that the distance between $z$ and $x$ is at most equal to the distance between $z$ and any other point of $\mathcal{X}$. The collection of all $C(x, \mathcal{X})$ with $x \in \mathcal{X}$ is called the Voronoi tessellation of $\mathbb{X}$. The Voronoi approximation of $A$ with respect to $\mathcal{X}$ is the union of all Voronoi cells $C(x, \mathcal{X}), x \in \mathcal{X}$, with $x \in A$, that is,

$$
A(\mathcal{X}):=\bigcup_{x \in \mathcal{X} \cap A} C(x, \mathcal{X})
$$

In the following, we let $\mathcal{X}$ be either a Poisson point process $\mathcal{P}_{s}, s \geq 1$, with intensity measure $s \mathbb{Q}$ or a binomial point process $\mathcal{X}_{n}$ of $n \in \mathbb{N}$ points distributed according to $\mathbb{Q}$. We are now interested in the behavior of the random approximations

$$
A_{s}:=A\left(\mathcal{P}_{s}\right), \quad s \geq 1, \quad \text { and } \quad A_{n}^{\prime}:=A\left(\mathcal{X}_{n}\right), \quad n \in \mathbb{N},
$$

of $A$. Note that $A_{s}$ is also called the Poisson-Voronoi approximation.

Typically, $A$ is an unknown set having unknown geometric characteristics such as volume and surface area. Notice that $A_{s}$ and $A_{n}^{\prime}$ are random polyhedral approximations of $A$, with volumes closely approximating that of $A$ as $s$ and $n$ become large. There is a large literature devoted to quantifying this approximation and we refer to $[20,48]$ for further discussion and references. One might also expect that $\mathcal{H}^{d-1}\left(\partial A_{s}\right)$ closely approximates a scalar multiple of $\mathcal{H}^{d-1}(\partial A)$, provided the latter quantity exists and is finite. This has been shown in [48]. Using Theorem 2.3(b), 
we deduce rates of normal convergence for the volume and surface area statistics of $A_{s}$ and $A_{n}^{\prime}$ as well as $\operatorname{Vol}\left(A_{s} \Delta A\right)$ and $\operatorname{Vol}\left(A_{n}^{\prime} \Delta A\right)$. Here and elsewhere in this section, we abbreviate $\operatorname{Vol}_{d}$ by Vol. The symmetric difference $U \Delta V$ of two sets $U, V \subset \mathbb{R}^{d}$ is given by $U \Delta V:=(U \backslash V) \cup(V \backslash U)$.

THEOREM 3.4. (a) Let $A \subset(-1 / 2,1 / 2)^{d}$ be closed and such that $\partial$ A satisfies $\overline{\mathcal{M}}^{d-1}(\partial A)<\infty$ and contains $a(d-1)$-dimensional $C^{2}$-submanifold and let $F \in$ $\left\{\operatorname{Vol}, \operatorname{Vol}(\cdot \Delta A), \mathcal{H}^{d-1}(\partial \cdot)\right\}$. Then there is a constant $\tilde{C} \in(0, \infty)$ such that

$$
d_{K}\left(\frac{F\left(A_{s}\right)-\mathbb{E} F\left(A_{s}\right)}{\sqrt{\operatorname{Var} F\left(A_{s}\right)}}, N\right) \leq \tilde{C} s^{-\frac{(d-1)}{2 d}}, \quad s \geq 1,
$$

and

$$
d_{K}\left(\frac{F\left(A_{n}^{\prime}\right)-\mathbb{E} F\left(A_{n}^{\prime}\right)}{\sqrt{\operatorname{Var} F\left(A_{n}^{\prime}\right)}}, N\right) \leq \tilde{C} n^{-\frac{(d-1)}{2 d}}, \quad n \geq 9
$$

as well as

$$
d_{K}\left(\frac{\operatorname{Vol}\left(A_{s}\right)-\operatorname{Vol}(A)}{\sqrt{\operatorname{Var} \operatorname{Vol}\left(A_{S}\right)}}, N\right) \leq \tilde{C} s^{-\frac{(d-1)}{2 d}}, \quad s \geq 1,
$$

and

$$
d_{K}\left(\frac{\operatorname{Vol}\left(A_{n}^{\prime}\right)-\operatorname{Vol}(A)}{\sqrt{\operatorname{Var} \operatorname{Vol}\left(A_{n}^{\prime}\right)}}, N\right) \leq \tilde{C} n^{-\frac{(d-1)}{2 d}}, \quad n \geq 9 .
$$

(b) If $F=\mathrm{Vol}$ and $A \subset(-1 / 2,1 / 2)^{d}$ is compact and convex, then all of the above inequalities are in force.

REMARKS. (i) The bound (3.8) provides a rate of convergence for the main result of [42] (see Theorem 1.1 there), which establishes asymptotic normality for $\operatorname{Vol}\left(A_{S}\right), A$ convex. The bound (3.8) also improves upon Corollary 2.1 of [48] which shows

$$
d_{K}\left(\frac{\operatorname{Vol}\left(A_{s}\right)-\mathbb{E} \operatorname{Vol}\left(A_{s}\right)}{\sqrt{\operatorname{Var} \operatorname{Vol}\left(A_{s}\right)}}, N\right)=O\left((\log s)^{3 d+1} s^{-\frac{(d-1)}{2 d}}\right) .
$$

Recall that the normal convergence of $\mathcal{H}^{d-1}\left(\partial A_{S}\right)$ is given in Remark (i) after Theorem 2.4 of [48] and the bound (3.8) for $F=\mathcal{H}^{d-1}(\partial \cdot)$ provides a rate for this normal convergence.

(ii) The bound (3.11) improves upon the bound of Theorem 6.1 of [20], which contains extra logarithmic factors, and thus addresses an open problem raised in Remark 6.9 of [20].

(iii) We may likewise deduce identical rates of normal convergence for other geometric statistics of $A_{s}$, including the total number of $k$-dimensional faces of $A_{s}$, $k \in\{0,1, \ldots, d-1\}$, as well as the $k$-dimensional Hausdorff measure of the union of the $k$-dimensional faces of $A_{s}$ [thus when $k=d-1$, this gives $\mathcal{H}^{d-1}\left(\partial A_{s}\right)$ ]. 
Second-order asymptotics, including the requisite variance lower bounds for these statistics, are established in [46]. In the case of geometric statistics of $A_{n}^{\prime}$, we expect similar variance lower bounds and central limit theorems.

(iv) Lower bounds for $\operatorname{Var} F\left(A_{s}\right)$ and $\operatorname{Var} F\left(A_{n}^{\prime}\right)$ are essential to showing (3.8)(3.11). We expect the order of these bounds to be unchanged if $\mathbb{Q}$ has a density bounded away from zero and infinity. We thus expect Theorem 3.5 to remain valid in this context because all other arguments in our proof hold for such $\mathbb{Q}$.

Proof OF TheOREM 3.4. We first prove (3.8) for $F=$ Vol and $F=$ $\operatorname{Vol}(\cdot \Delta A)$. The proof method extends easily to the case when Poisson input is replaced by binomial input and we sketch the details as needed. To deduce (3.8) from Theorem 2.3(b), we need to (i) express $s F\left(A_{s}\right)$ as a sum of stabilizing score functions and (ii) define $K \subset \mathbb{X}$ and show that the scores decay exponentially fast with respect to $K$.

(i) Definition of scores. As in [48], for $\mathcal{X} \in \mathbf{N}, x \in \mathcal{X}$, and a fixed subset $A$ of $\mathbb{X}$, define the scores

$$
v^{ \pm}(x, \mathcal{X}):= \begin{cases}\operatorname{Vol}\left(C(x, \mathcal{X}) \cap A^{c}\right) & \text { if } x \in A, \\ \pm \operatorname{Vol}(C(x, \mathcal{X}) \cap A) & \text { if } x \in A^{c} .\end{cases}
$$

Define $v_{s}^{ \pm}(x, \mathcal{X}):=s v^{ \pm}(x, \mathcal{X})$. By the definition of $v^{ \pm}$at (3.12), we have

$$
s \operatorname{Vol}\left(A_{s}\right)=\sum_{x \in \mathcal{P}_{s}} v_{s}^{-}\left(x, \mathcal{P}_{s}\right)+s \operatorname{Vol}(A)
$$

and

$$
s \operatorname{Vol}\left(A \Delta A_{S}\right)=\sum_{x \in \mathcal{P}_{s}} v_{s}^{+}\left(x, \mathcal{P}_{S}\right) .
$$

The arguments of Section 5.1 of [26] show that the scores $v_{s}^{ \pm}$have a radius of stabilization $R_{s}\left(x, \mathcal{P}_{s} \cup\{x\}\right)$ with respect to $\mathcal{P}_{s}$ which satisfies (2.4) with $\gamma=d$ and $\alpha_{\text {stab }}=d$. The scores $v_{s}^{ \pm}$also satisfy the $(4+p)$ th moment condition (2.7) for all $p \in[0, \infty)$.

As remarked in [48] and as shown in Lemma 5.1 of [26], the scores $v_{n}^{ \pm}$have a radius of stabilization $R_{n}\left(x, \mathcal{X}_{n-8} \cup\{x\}\right)$ with respect to binomial input $\mathcal{X}_{n}$ which satisfies (2.5) with $\gamma=d$ and $\alpha_{\text {stab }}=d$.

(ii) Definition of $K$. We set $K$ to be $\partial A$. As noted in the proof of Theorem 2.1 of [48], we assert that the scores $v_{s}^{ \pm}$decay exponentially fast with their distance to $\partial A$, that is, they satisfy (2.8) and (2.9) when $K$ is set to $\partial A$ and with $\alpha_{K}=d$. To see this for Poisson input, note that

$$
\mathbb{P}\left(v_{s}^{ \pm}\left(x, \mathcal{P}_{s} \cup\{x\}\right) \neq 0\right) \leq \mathbb{P}\left(\operatorname{diam}\left(C\left(x, \mathcal{P}_{s} \cup\{x\}\right)\right) \geq \mathrm{d}(x, K)\right)
$$

for $x \in[-1 / 2,1 / 2]^{d}$. Since $\operatorname{diam}\left(C\left(x, \mathcal{P}_{S} \cup\{x\}\right)\right) \leq 2 R_{S}\left(x, \mathcal{P}_{S} \cup\{x\}\right)$ and since $R_{S}\left(x, \mathcal{P}_{S} \cup\{x\}\right)$ has exponentially decaying tails, the assertion follows. 
We deduce (3.8) from the bound (2.18) of Theorem 2.3(b) as follows. If either $\partial A$ contains a $(d-1)$-dimensional $C^{2}$-submanifold or $A$ is compact and convex, then $s^{2} \operatorname{Var} \operatorname{Vol}\left(A_{s}\right)=\Omega\left(s^{(d-1) / d}\right)$; see Theorem 1.2 of [42], Theorem 1.1 of [46] and Theorem 2.2 of [48]. All conditions of Theorem 2.3 are satisfied and so (3.8) follows for $F=\operatorname{Vol}$. Replacing $\operatorname{Vol}\left(A_{s}\right)$ with $\operatorname{Vol}\left(A \Delta A_{s}\right)$, (3.8) holds if $\partial A$ contains a $(d-1)$-dimensional $C^{2}$-submanifold. This assertion follows since the stated conditions imply $s^{2} \operatorname{Var} \operatorname{Vol}\left(A \Delta A_{s}\right)=\Omega\left(s^{(d-1) / d}\right)$, as shown in Theorem 2.2 of [48]. We may similarly deduce (3.9) from the bound (2.18) of Theorem 2.3(b). If either $\partial A$ contains a $(d-1)$-dimensional $C^{2}$-submanifold or $A$ is compact and convex, then $n^{2} \operatorname{Var} \operatorname{Vol}\left(A_{n}^{\prime}\right)=\Omega\left(n^{(d-1) / d}\right)$ as shown in Theorem 2.3 of [48]. Thus (3.9) follows for $F=\operatorname{Vol}$. Considering now $F=\operatorname{Vol}(\cdot \Delta A)$, and appealing to the variance lower bounds of Theorem 2.3 of [48], we see that when $\partial A$ contains a $(d-1)$-dimensional $C^{2}$-submanifold, all conditions of Theorem 2.3(b) are satisfied in the context of binomial input, and so the bound (3.9) follows for $F=\operatorname{Vol}(\cdot \Delta A)$.

To deduce (3.10) from (3.8), we need to replace $\mathbb{E} \operatorname{Vol}\left(A_{s}\right)$ with $\operatorname{Vol}(A)$. As shown in [21], Theorem 2, if the random input consists of $n$ i.i.d. uniformly distributed random variables then $\left|\mathbb{E} \operatorname{Vol}\left(A_{n}^{\prime}\right)-\operatorname{Vol}(A)\right| \leq c^{n}$ for some $c \in(0,1)$. A similar statement holds for Poisson input $\mathcal{P}_{s}$ : If $\left|\mathcal{P}_{s}\right|$ is the cardinality of $\mathcal{P}_{s}$, then

$$
\begin{aligned}
\left|\mathbb{E} \operatorname{Vol}\left(A_{s}\right)-\operatorname{Vol}(A)\right| & =\sum_{n \in \mathbb{N}} \mathbb{P}\left(\left|\mathcal{P}_{s}\right|=n\right)\left|\mathbb{E} \operatorname{Vol}\left(A_{n}^{\prime}\right)-\operatorname{Vol}(A)\right| \\
& \leq \exp (s(c-1)) .
\end{aligned}
$$

This exponential bias allows one to replace $\mathbb{E} \operatorname{Vol}\left(A_{s}\right)$ by $\operatorname{Vol}(A)$ in $(3.8)$ and similarly for $\mathbb{E} \operatorname{Vol}\left(A_{n}^{\prime}\right)$. This gives (3.10) and (3.11).

We now show (3.8) for $F=\mathcal{H}^{d-1}(\partial \cdot)$ and that it also holds when Poisson input is replaced by binomial input. Given $\mathcal{X} \in \mathbf{N}$, define for $x \in \mathcal{X} \cap A$ the score $\alpha(x, \mathcal{X})$ to be the $\mathcal{H}^{d-1}$ measure of the $(d-1)$-dimensional faces of $C(x, \mathcal{X})$ belonging to the boundary of $\bigcup_{w \in \mathcal{X} \cap A} C(w, \mathcal{X})$; if there are no such faces or if $x \notin \mathcal{X} \cap A$, then set $\alpha(x, \mathcal{X})$ to be zero.

Put $\alpha_{s}(x, \mathcal{X}):=s^{(d-1) / d} \alpha(x, \mathcal{X})$. Recalling the notation in (1.1) and (1.2), the surface area of $A_{s}$ and $A_{n}^{\prime}$ is then given by

$$
{ }_{s}^{(d-1) / d} \mathcal{H}^{d-1}\left(\partial A_{s}\right)=h_{s}\left(\mathcal{P}_{s}\right)=\sum_{x \in \mathcal{P}_{s}} \alpha_{s}\left(x, \mathcal{P}_{s}\right)
$$

and

$$
n^{(d-1) / d} \mathcal{H}^{d-1}\left(\partial A_{n}^{\prime}\right)=h_{n}\left(\mathcal{X}_{n}\right)=\sum_{x \in \mathcal{X}_{n}} \alpha_{n}\left(x, \mathcal{X}_{n}\right),
$$

respectively. We want to deduce (3.8) and (3.9) for $F=\mathcal{H}^{d-1}(\partial \cdot)$ from Theorem 2.3(b) with $K$ set to $\partial A$. As shown in the proof of Theorem 2.5 of [48], the 
scores $\alpha_{s}$ are exponentially stabilizing with respect to Poisson and binomial input. In other words, they satisfy (2.4) and (2.5) with $\gamma=d$ and $\alpha_{\text {stab }}=d$. They also satisfy the $(4+p)$ th moment conditions $(2.6)$ and (2.7) for all $p \in[0, \infty)$. As noted in the proof of Theorem 2.5 of [48], the scores $\alpha_{s}$ decay exponentially fast with their distance to $\partial A$, that is, they satisfy (2.8) and (2.9) when $K$ is set to $\partial A$. We note that

$$
\operatorname{Var} \mathcal{H}^{d-1}\left(\partial A_{s}\right)=\Theta\left(s^{-(d-1) / d}\right),
$$

as shown in Theorem 1.1 of [46]. We assert that

$$
\operatorname{Var} \mathcal{H}^{d-1}\left(\partial A_{n}^{\prime}\right)=\Theta\left(n^{-(d-1) / d}\right) .
$$

This may be proved by mimicking the methods to prove (3.13) or, alternatively, with $Z(n)$ denoting an independent Poisson random variable with mean $n$, we could use Lemma 6.1 of [48] to show $\left|\operatorname{Var} h_{n}\left(\mathcal{X}_{Z(n)}\right)-\operatorname{Var} h_{n}\left(\mathcal{X}_{n}\right)\right|=o\left(n^{(d-1) / d}\right)$. Hence, all conditions of Theorem 2.3(b) are satisfied for Poisson and for binomial input. This gives (3.8) and (3.9) for $F=\mathcal{H}^{d-1}(\partial \cdot)$, as desired.

3.4. Statistics of convex hulls of random point samples. In the following, let $A$ be a compact convex subset of $\mathbb{R}^{d}$ with nonempty interior, $C^{2}$-boundary and positive Gaussian curvature. By $\mathbb{Q}$, we denote the uniform measure on $A$. Let $\mathcal{P}_{s}$, $s \geq 1$, be a Poisson point process with intensity measure $s \mathbb{Q}$ and let $\mathcal{X}_{n}, n \in \mathbb{N}$, be a binomial point process of $n$ independent points distributed according to $\mathbb{Q}$. From now on, $\operatorname{Conv}(\mathcal{X})$ stands for the convex hull of a set $\mathcal{X} \subset \mathbb{R}^{d}$. The aim of this subsection is to establish rates of normal convergence for statistics of the random polytopes $\operatorname{Conv}\left(\mathcal{P}_{S}\right)$ and $\operatorname{Conv}\left(\mathcal{X}_{n}\right)$. We denote the number of $k$-faces of a polytope $P$ by $f_{k}(P), k \in\{0, \ldots, d-1\}$, and its intrinsic volumes by $V_{i}(P), i \in\{1, \ldots, d\}$.

THEOREM 3.5. For any $h \in\left\{f_{0}, \ldots, f_{d-1}, V_{1}, \ldots, V_{d}\right\}$, there is a constant $C_{h} \in(0, \infty)$ also depending on $A$ such that

$$
d_{K}\left(\frac{h\left(\operatorname{Conv}\left(\mathcal{P}_{s}\right)\right)-\mathbb{E} h\left(\operatorname{Conv}\left(\mathcal{P}_{s}\right)\right)}{\sqrt{\operatorname{Var} h\left(\operatorname{Conv}\left(\mathcal{P}_{S}\right)\right)}}, N\right) \leq C_{h} s^{-\frac{d-1}{2(d+1)}}, \quad s \geq 1,
$$

and

$$
\begin{aligned}
d_{K}\left(\frac{h\left(\operatorname{Conv}\left(\mathcal{X}_{n}\right)\right)-\mathbb{E} h\left(\operatorname{Conv}\left(\mathcal{X}_{n}\right)\right)}{\sqrt{\operatorname{Var} h\left(\operatorname{Conv}\left(\mathcal{X}_{n}\right)\right)}}, N\right) \\
\quad \leq C_{h} n^{-\frac{d-1}{2(d+1)}}, \quad n \geq \max \{9, d+2\}
\end{aligned}
$$

REMARKS. (i) Previous work. The asymptotic study of the statistics $h\left(\operatorname{Conv}\left(\mathcal{P}_{s}\right)\right)$ and $h\left(\operatorname{Conv}\left(\mathcal{X}_{n}\right)\right), h \in\left\{f_{0}, \ldots, f_{d-1}, V_{1}, \ldots, V_{d}\right\}$, has a long and rich history, starting with the seminal works [36, 37]. The breakthrough paper [34], which relies on dependency graph methods and Voronoi cells, establishes 
rates of normal convergence for Poisson input and $h \in\left\{f_{0}, \ldots, f_{d-1}, V_{d}\right\}$ of the order $s^{-\frac{d-1}{2(d+1)}}$ times some power of $\log s$ (see Theorems 1 and 2). Still in the setting $h \in\left\{f_{0}, \ldots, f_{d-1}, V_{d}\right\}$, but with binomial input Theorem 1.2 and Theorem 1.3 of [47] provide the rates of convergence $n^{-1 /(d+1)+o(1)}$ for $d \geq 3$ and $n^{-1 / 6+o(1)}$ for $d=2$, which improved previous bounds in [34] for the binomial case, but is still weaker than (3.15). When $h \in\left\{f_{0}, \ldots, f_{d-1}, V_{1}, \ldots, V_{d}\right\}$ and $A$ is the unit ball, Theorem 7.1 of [9] gives a central limit theorem for $h\left(\operatorname{Conv}\left(\mathcal{P}_{S}\right)\right)$, with convergence rates involving extra logarithmic factors. Central limit theorems for intrinsic volume functionals over binomial input were derived recently (in parallel and independently of us) in [45]. There, the rate of convergence is only for the Wasserstein distance and contains the additional factor $(\log n)^{3+2 /(d+1)}$ compared to (3.15).

(ii) Extensions. Lower bounds for $\operatorname{Var} h\left(\operatorname{Conv}\left(\mathcal{P}_{s}\right)\right)$ and $\operatorname{Var} h\left(\operatorname{Conv}\left(\mathcal{X}_{n}\right)\right)$ are essential to showing (3.14) and (3.15). We expect the order of these bounds to be unchanged if $\mathbb{Q}$ has a density bounded away from zero and infinity. Consequently, we anticipate that Theorem 3.5 remains valid in this context because all other arguments in our proof below also work for such a density.

In the following, we may assume without loss of generality that $\mathbf{0}$ is in the interior of $A$. The proof of Theorem 3.5 is divided into several lemmas and we prepare it by recalling some geometric facts and introducing some notation.

For a boundary point $z \in \partial A$, we denote by $T_{z}$ the tangent space parametrized by $\mathbb{R}^{d-1}$ in such a way that $z$ is the origin. The boundary of $A$ in a neighborhood of $z$ may be identified with the graph of a function $f_{z}: T_{z} \rightarrow \mathbb{R}$. It may be deduced from [34], Section 5, that there are constants $\underline{c} \in(0,1), \bar{c} \in(1, \infty)$ and $r_{0} \in(0, \infty)$ such that uniformly for all $z \in \partial A$,

$$
\underline{c}^{2}\|v\|^{2} \leq f_{z}(v) \leq \bar{c}^{2}\|v\|^{2}, \quad v \in T_{z} \cap B^{d-1}\left(\mathbf{0}, r_{0}\right),
$$

where we denote by $B^{m}(x, r)$ the closed ball with center $x \in \mathbb{R}^{m}$ and radius $r>0$ in $\mathbb{R}^{m}, m \in \mathbb{N}$.

For $u>0$, we define

$$
A_{-u}:=\left\{y \in A: \mathrm{d}\left(y, A^{c}\right) \leq u\right\},
$$

where $A^{c}:=\mathbb{R}^{d} \backslash A$. It follows from (3.16) that there is a $\varrho>0$ such that all points $x \in A_{-3 \varrho}$ have a unique projection $\prod_{\partial A}(x)$ to $\partial A$. For $3 \varrho \geq \bar{r} \geq r \geq \underline{r} \geq 0$, it also holds that

$$
\begin{aligned}
& \partial A_{-\bar{r}} \subset\left(\partial A_{-r} \oplus(\bar{r}-r) B^{d}(0,1)\right) \quad \text { and } \\
& \partial A_{-\underline{r}} \subset\left(\partial A_{-r} \oplus(r-\underline{r}) B^{d}(0,1)\right) .
\end{aligned}
$$

We denote by $d_{\max }$ the metric

$$
\mathrm{d}_{\max }(x, y):=\max \left\{\|x-y\|, \sqrt{\left|\mathrm{d}\left(x, A^{c}\right)-\mathrm{d}\left(y, A^{c}\right)\right|}\right\}, \quad x, y \in A,
$$


and define for $x \in A$ and $r>0$,

$$
B_{\mathrm{d}_{\max }}(x, r):=\left\{y \in A: \mathrm{d}_{\max }(x, y) \leq r\right\} .
$$

The following lemma ensures that the space $(A, \mathcal{B}(A), \mathbb{Q})$ and the metric $\mathrm{d}_{\text {max }}$ satisfy condition (2.1) for $x \in A_{-\varrho}$, with $\gamma=d+1$.

LEMMA 3.6. There is a constant $\kappa>0$ such that for all $x \in A_{-\varrho}$ and $r>0$

$$
\limsup _{\varepsilon \rightarrow 0} \frac{\mathbb{Q}\left(B_{\mathrm{d}_{\max }}(x, r+\varepsilon)\right)-\mathbb{Q}\left(B_{\mathrm{d}_{\max }}(x, r)\right)}{\varepsilon} \leq \kappa(d+1) r^{d} \text {. }
$$

PROOF. Recall that, for $w>0$ and $D \subset \mathbb{R}^{d}$, the outer $w$-parallel set of $D$ is $\left\{x \in D^{c}: \mathrm{d}(x, D) \leq w\right\}$. For $u, v \geq 0, A_{-(u+v)} \backslash A_{-u}=\left(A \backslash A_{-u}\right)_{-v}$ and $A \backslash A_{-u}$ is convex. Consequently, $\mathbb{Q}\left(\left(A \backslash A_{-u}\right)_{-v}\right)$ can be bounded by the volume of the outer $v$-parallel set of $A \backslash A_{-u}$, which can be bounded by the volume of the outer $v$-parallel set of $A$ so that

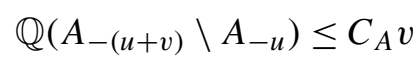

with some universal constant $C_{A}$ only depending on $A$. Since a similar inequality holds for $\mathbb{Q}\left(B^{d}(x, u+v) \backslash B^{d}(x, u)\right)$, we see that the lim sup in (3.18) is bounded. For this reason, it is sufficient to establish (3.18) for small $r$.

We define for $x \in A_{-\varrho}$ and $r \in(0, \varrho)$,

$$
U_{x, r}:=B_{\mathrm{d}_{\max }}(x, r) \cap\{y \in A:\|x-y\|=r\}
$$

and

$$
V_{x, r}:=B_{\mathrm{d}_{\max }}(x, r) \cap\left\{y \in A:\left|\mathrm{d}\left(x, A^{c}\right)-\mathrm{d}\left(y, A^{c}\right)\right|=r^{2}\right\} .
$$

It follows from (3.17) that

$$
\begin{aligned}
& \limsup _{\varepsilon \rightarrow 0} \frac{\mathbb{Q}\left(B_{\mathrm{d}_{\max }}(x, r+\varepsilon)\right)-\mathbb{Q}\left(B_{\mathrm{d}_{\max }}(x, r)\right)}{\varepsilon} \\
& \quad \leq \limsup _{\varepsilon \rightarrow 0} \frac{\mathbb{Q}\left(U_{x, r} \oplus|\varepsilon| B^{d}(0,1)\right)+\mathbb{Q}\left(V_{x, r} \oplus\left(2 r|\varepsilon|+\varepsilon^{2}\right) B^{d}(0,1)\right)}{|\varepsilon|} \\
& \quad \leq 2 \mathcal{H}^{d-1}\left(U_{x, r}\right)+4 r \mathcal{H}^{d-1}\left(V_{x, r}\right) .
\end{aligned}
$$

For $r$ sufficiently small, we obtain sub- and supersets for $A_{-\left(\mathrm{d}\left(x, A^{c}\right)-r^{2}\right)} \cap B^{d}(x, r)$ and $A_{-\left(\mathrm{d}\left(x, A^{c}\right)+r^{2}\right)} \cap B^{d}(x, r)$ by taking the inner parallel sets with respect to the paraboloids given in (3.16). Consequently, $U_{x, r}$ is contained in a strip whose Euclidean thickness is of the order $r^{2}$. This implies that $\mathcal{H}^{d-1}\left(U_{x, r}\right) \leq c_{A} r^{d}$ for all $r>0$ with some constant $c_{A} \in(0, \infty)$ only depending on $A$. Since $V_{x, r}$ is the union of the intersection of the boundaries of the convex sets $A_{-\left(\mathrm{d}\left(x, A^{c}\right)+r^{2}\right)}$ and 
$A_{-\left(\mathrm{d}\left(x, A^{c}\right)-r^{2}\right)}$ with $B^{d}(x, r)$, we have that $\mathcal{H}^{d-1}\left(V_{x, r}\right) \leq 2 d \kappa_{d} r^{d-1}$, which completes the proof.

We let $u_{x}:=\left(\prod_{\partial A}(x)-x\right) /\left\|\prod_{\partial A}(x)-x\right\|$ for $x \in \operatorname{int}(A)$, whereas for $x \in$ $\partial A$ we let $u_{x}$ be the outer unit normal at $x$. For $x \in A$ and $r>0$, we define the hyperplanes

$$
H_{x}:=\left\{y \in \mathbb{R}^{d}:\left\langle u_{x}, y\right\rangle=\left\langle u_{x}, x\right\rangle\right\}
$$

and the parametrized family of sets

$$
A_{x, r}:= \begin{cases}\operatorname{Conv}\left(\left(H_{x} \cap B^{d}(x, r / \bar{c})\right) \cup\left\{x+r^{2} u_{x}\right\}\right) & \text { if } r \leq \sqrt{\mathrm{d}\left(x, A^{c}\right)}, \\ A \backslash \operatorname{Conv}\left(\left(A \backslash B^{d}(x, r / \underline{c})\right) \cup\{x\}\right) & \text { if } r>\sqrt{\mathrm{d}\left(x, A^{c}\right)} .\end{cases}
$$

When $x \in A_{-\varrho}$ and $r>\sqrt{\mathrm{d}\left(x, A^{c}\right)}$ is sufficiently small, we note that $x$ is an extreme point of $A \backslash A_{x, r}$. The sets $A_{x, r}$ have the following important properties.

LEMMA 3.7. (a) There are constants $C_{\mathbb{Q}}, c_{\mathbb{Q}} \in(0, \infty)$ such that

$$
C_{\mathbb{Q}} r^{d+1} \geq \mathbb{Q}\left(A_{x, r}\right) \geq c_{\mathbb{Q}} r^{d+1}, \quad x \in A_{-\varrho}, r \in[0,1] .
$$

(b) There is a constant $c_{\max } \in(0, \infty)$ such that $A_{x, r} \subset B_{\mathrm{d}_{\max }}\left(x, c_{\max } r\right)$ for any $r>0$ and $x \in A_{-\tilde{\varrho}}$ with $\tilde{\varrho}:=\min \left\{1 /\left(4 \bar{c}^{2}\right), \varrho\right\}$.

Proof. We denote the epigraphs of $v \mapsto \underline{c}^{2}\|v\|^{2}$ and $v \mapsto \bar{c}^{2}\|v\|^{2}$ by $\underline{P}_{z}$ and $\bar{P}_{z}$. For $r \leq \sqrt{\mathrm{d}\left(x, A^{c}\right)}$ we have $\mathbb{Q}\left(A_{x, r}\right)=\kappa_{d-1} r^{d+1} /\left(d \bar{c}^{d-1}\right)$. For $x \in A$ and $r>\sqrt{\mathrm{d}\left(x, A^{c}\right)}$ let $z:=\prod_{\partial A}(x)$. Since

$$
\operatorname{Conv}\left(\left(A \backslash B^{d}(x, r / \underline{c})\right) \cup\{x\}\right) \subset \operatorname{Conv}\left(\left(A \backslash B^{d}(z, r / \underline{c})\right) \cup\{z\}\right),
$$

it follows that $A_{x, r} \supset A_{z, r}$. Additionally,

$$
A_{z, r} \supset \bar{P}_{z} \backslash \operatorname{Conv}\left(\{z\} \cup\left(\underline{P}_{z} \backslash B^{d}(z, r / \underline{c})\right)\right) .
$$

A longer computation shows that the volume of the set on the right-hand side can be bounded below by a nonnegative scalar multiple of $r^{d+1}$. The upper bound in part (a) can be proven similarly.

To prove part (b), it suffices to consider only the situation $r \in[0,1]$. It follows immediately from the definition of $A_{x, r}$ that $A_{x, r} \subset B^{d}(x, r / \underline{c})$ for $r \in[0,1]$. For $x \in A_{-\varrho}$ with $\mathrm{d}\left(x, A^{c}\right) \leq 1 /\left(4 \bar{c}^{2}\right), r \leq \sqrt{\mathrm{d}\left(x, A^{c}\right)}$ and $y \in A_{x, r}$, we obtain by a direct but longer computation that

$$
\mathrm{d}\left(x, A^{c}\right) \geq \mathrm{d}\left(y, A^{c}\right) \geq \mathrm{d}\left(x, A^{c}\right)-4 \bar{c}^{2} r^{2} .
$$

On the other hand, for $r>\sqrt{\mathrm{d}\left(x, A^{c}\right)}$ and $y \in A_{x, r}$, we have with $z=\prod_{\partial A}(x)$ that

$$
\begin{aligned}
\mathrm{d}\left(y, A^{c}\right) & \leq \sup _{v \in \partial A \cap B^{d}(x, r / \underline{c})} \mathrm{d}\left(v, H_{z}\right) \leq \sup _{v \in \bar{P}_{z} \cap B^{d}\left(z, r / \underline{c}+\mathrm{d}\left(x, A^{c}\right)\right)} \mathrm{d}\left(v, H_{z}\right) \\
& \leq \sup _{v \in \bar{P}_{z} \cap B^{d}(z,(1 / \underline{c}+1) r)} \mathrm{d}\left(v, H_{z}\right) \leq \bar{c}^{2}(1 / \underline{c}+1)^{2} r^{2} .
\end{aligned}
$$


This implies that $A_{x, r} \subset\left\{y \in B^{d}(0,1): \sqrt{\left|\mathrm{d}\left(x, A^{c}\right)-\mathrm{d}\left(y, A^{c}\right)\right|} \leq \bar{c}(1 / \underline{c}+2) r\right\}$, which completes the proof of part (b).

For $k \in\{0, \ldots, d-1\}$ and $\mathcal{X} \in \mathbf{N}$, let $\mathcal{F}_{k}(\operatorname{Conv}(\mathcal{X}))$ be the set of $k$-dimensional faces of $\operatorname{Conv}(\mathcal{X})$. To cast $f_{k}(\operatorname{Conv}(\mathcal{X}))$ in the form of (1.1) and (1.2), we define

$$
\xi_{k}(x, \mathcal{X}):=\frac{1}{k+1} \sum_{F \in \mathcal{F}_{k}(\operatorname{Conv}(\mathcal{X}))} \mathbf{1}_{\{x \in F\}}, \quad x \in \mathcal{X} .
$$

Note that $f_{k}(\operatorname{Conv}(\mathcal{X}))=\sum_{x \in \mathcal{X}} \xi_{k}(x, \mathcal{X})$.

To cast the intrinsic volumes $V_{j}(\operatorname{Conv}(\mathcal{X})), j \in\{1, \ldots, d-1\}$, in the form of (1.1) and (1.2), we need some more notation. Given the convex set $A$ and a linear subspace $E$, denote by $A \mid E$ the orthogonal projection of $A$ onto $E$. For $x \in \mathbb{R}^{d} \backslash\{0\}$, let $L(x)$ the line spanned by $x$. Given a line $N \subset \mathbb{R}^{d}$ through the origin, and for $1 \leq j \leq d$, let $G(N, j)$ be the set of $j$-dimensional linear subspaces of $\mathbb{R}^{d}$ containing $N$. Let then $v_{j}^{N}(\cdot)$ be the Haar probability measure on $G(N, j)$. Let $M \subset A$ be convex. For $j \in\{0, \ldots, d-1\}, x \in \mathbb{R}^{d} \backslash\{\mathbf{0}\}$, and $L \in G(L(x), j)$ define

$$
f^{L}(x):=\mathbf{1}_{\{x \in(A \mid L) \backslash(M \mid L)\}}
$$

and, as in [9], define the projection avoidance function $\theta_{j}^{A, M}: \mathbb{R}^{d} \backslash\{\mathbf{0}\} \mapsto[0,1]$ by

$$
\theta_{j}^{A, M}(x):=\int_{G(L(x), j)} f^{L}(x) v_{j}^{L(x)}(\mathrm{d} L) .
$$

The following result generalizes [9], (2.7), to nonspherical compact sets, with arguments similar to Lemma A1 from [16]. The proof is in the Appendix.

LEMMA 3.8. Let $M \subset A$ be a convex subset of $\mathbb{R}^{d}$. For all $j \in\{0, \ldots, d-1\}$ there is a constant $\kappa_{d, j}$ depending on $d, j$ such that

$$
V_{j}(A)-V_{j}(M)=\kappa_{d, j} \int_{A \backslash M} \theta_{j}^{A, M}(x)\|x\|^{-(d-j)} \mathrm{d} x .
$$

For $\mathcal{X} \in \mathbf{N}$ and $F \in \mathcal{F}_{d-1}(\operatorname{Conv}(\mathcal{X}))$ put cone $(F):=\{r y: y \in F, r>0\}$. Define for $j \in\{1, \ldots, d-1\}$

$$
\begin{aligned}
\xi_{j, s}(x, \mathcal{X}) & \\
= & \frac{s \kappa_{d, j}}{d} \\
& \times \sum_{F \in \mathcal{F}_{d-1}(\operatorname{Conv}(\mathcal{X}))} \mathbf{1}_{\{x \in F\}} \int_{\operatorname{Cone}(F) \cap(A \backslash \operatorname{Conv}(\mathcal{X}))}\|x\|^{-(d-j)} \theta_{j}^{A, \operatorname{Conv}(\mathcal{X})}(x) \mathrm{d} x
\end{aligned}
$$


for $x \in \mathcal{X}, s \geq 1$. Lemma 3.8 yields

$$
s\left(V_{j}(A)-V_{j}(\operatorname{Conv}(\mathcal{X}))\right)=\sum_{x \in \mathcal{X}} \xi_{j, s}(x, \mathcal{X})
$$

if $\mathbf{0}$ is in the interior of $\operatorname{Conv}(\mathcal{X})$ and if all points of $\mathcal{X}$ are in general position. For $x \in \mathcal{X}$ and $s \geq 1$, define

$$
\xi_{d, s}(x, \mathcal{X}):=\frac{s}{d} \sum_{F \in \mathcal{F}_{d-1}(\operatorname{Conv}(\mathcal{X}))} \mathbf{1}_{\{x \in F\}} \int_{\operatorname{Cone}(F) \cap(A \backslash \operatorname{Conv}(\mathcal{X}))} \mathrm{d} x .
$$

If $\mathbf{0}$ is in the interior of $\operatorname{Conv}(\mathcal{X})$ and all points of $\mathcal{X}$ are in general position, we have as well

$$
s V_{d}(A \backslash \operatorname{Conv}(\mathcal{X}))=\sum_{x \in \mathcal{X}} \xi_{d, s}(x, \mathcal{X})
$$

The definitions of the scores and $\left\|\theta_{j}^{A, \operatorname{Conv}(\mathcal{X})}\right\|_{\infty} \leq 1$ show that for $\mathcal{X} \in \mathbf{N}, x \in \mathcal{X}$, $s \geq 1$ and $j \in\{0, \ldots, d-1\}$

$$
\xi_{j, s}(x, \mathcal{X}) \leq \kappa_{d, j} r(\operatorname{Conv}(\mathcal{X}))^{-(d-j)} \xi_{d, s}(x, \mathcal{X}),
$$

where $r(\operatorname{Conv}(\mathcal{X}))$ is the radius of the largest ball centered at $\mathbf{0}$ and contained in $\operatorname{Conv}(\mathcal{X})$.

Since $\mathbf{0} \in \operatorname{int}(A)$, we can choose $\rho_{0} \in(0, \tilde{\varrho})$ such that $B\left(\mathbf{0}, 2 \rho_{0}\right) \subset A$. For a score $\xi$, we denote by $\tilde{\xi}$ the modified score

$$
\tilde{\xi}(x, \mathcal{X}):=\mathbf{1}_{\left\{x \in A_{-\rho_{0}}\right\}} \xi\left(x,\left(\mathcal{X} \cap A_{-\rho_{0}}\right) \cup\{\mathbf{0}\}\right)
$$

for $\mathcal{X} \in \mathbf{N}$ and $x \in \mathcal{X}$. Our strategy of proof for Theorem 3.5 is to apply in a first step Corollary 2.2 in connection with Remark (vii) after Theorem 2.3 to these modified scores, putting $\mathbb{X}:=A$ and $\tilde{\mathbb{X}}:=A_{-\rho_{0}}$ and $K$ set to $\partial A$. Thereafter, we show that the result remains true without truncating and without adding the origin as an additional point.

For a score $\xi$ and $\mathcal{X} \in \mathbf{N}$, we define

$$
S_{\xi}(\mathcal{X}):=\sum_{x \in \mathcal{X}} \xi(x, \mathcal{X})
$$

LEMMA 3.9. For any $\xi_{s} \in\left\{\xi_{0}, \ldots, \xi_{d-1}, \xi_{1, s}, \ldots, \xi_{d, s}\right\}$, there are constants $C_{0}, c_{0} \in(0, \infty)$ such that

$$
\begin{aligned}
& \max \left\{\mathbb{P}\left(S_{\xi_{s}}\left(\mathcal{P}_{s}\right) \neq S_{\tilde{\xi}_{s}}\left(\mathcal{P}_{s}\right)\right), \mathbb{P}\left(B^{d}\left(\mathbf{0}, \rho_{0}\right) \not \subset \operatorname{Conv}\left(\mathcal{P}_{s}\right)\right),\left|\mathbb{E} S_{\xi_{s}}\left(\mathcal{P}_{s}\right)-\mathbb{E} S_{\tilde{\xi}_{s}}\left(\mathcal{P}_{s}\right)\right|,\right. \\
& \left.\left|\operatorname{Var} S_{\xi_{s}}\left(\mathcal{P}_{s}\right)-\operatorname{Var} S_{\tilde{\xi}_{s}}\left(\mathcal{P}_{s}\right)\right|\right\} \\
& \quad \leq C_{0} \exp \left(-c_{0} s\right)
\end{aligned}
$$


for $s \geq 1$ and

$$
\begin{aligned}
& \max \left\{\mathbb{P}\left(S_{\xi_{n}}\left(\mathcal{X}_{n}\right) \neq S_{\tilde{\xi}_{n}}\left(\mathcal{X}_{n}\right)\right), \mathbb{P}\left(B^{d}\left(\mathbf{0}, \rho_{0}\right) \not \subset \operatorname{Conv}\left(\mathcal{X}_{n}\right)\right),\right. \\
&\left.\left|\mathbb{E} S_{\xi_{n}}\left(\mathcal{X}_{n}\right)-\mathbb{E} S_{\tilde{\xi}_{n}}\left(\mathcal{X}_{n}\right)\right|,\left|\operatorname{Var} S_{\xi_{n}}\left(\mathcal{X}_{n}\right)-\operatorname{Var} S_{\tilde{\xi}_{n}}\left(\mathcal{X}_{n}\right)\right|\right\} \\
& \quad \leq C_{0} \exp \left(-c_{0} n\right)
\end{aligned}
$$

for $n \geq 1$.

Proof. One can choose sets $A_{1}, \ldots, A_{m} \subset\left\{x \in A: \mathrm{d}\left(x, A^{c}\right) \leq \rho_{0}\right\}$ with nonempty interior such that, for any $\mathcal{X} \in \mathbf{N}$ with $A_{i} \cap \mathcal{X} \neq \varnothing, i \in\{1, \ldots, m\}$,

$$
\operatorname{conv}(\mathcal{X}) \supset\left\{x \in A: \mathrm{d}\left(x, A^{c}\right)>\rho_{0}\right\} .
$$

Using $B\left(\mathbf{0}, 2 \rho_{0}\right) \subset A$, this inclusion yields $B\left(\mathbf{0}, \rho_{0}\right) \subset \operatorname{Conv}(\mathcal{X})$. The event $S_{\tilde{\xi}_{s}}(\mathcal{X}) \neq S_{\xi_{s}}(\mathcal{X})$ is also a subset of the event $A_{i} \cap \mathcal{X}=\varnothing$ for some $i \in\{1, \ldots, m\}$. These observations prove the probability bounds.

The generous upper bounds

$\max _{k \in\{0, \ldots, d-1\}} f_{k}(\operatorname{conv}(\mathcal{X})) \leq|\mathcal{X}|^{d-1}$ and $\max _{i \in\{1, \ldots, d\}} V_{i}(\operatorname{conv}(\mathcal{X})) \leq \max _{i \in\{1, \ldots, d\}} V_{i}(A)$

lead to $\left|S_{\tilde{\xi}_{s}}(\mathcal{X})-S_{\xi_{s}}(\mathcal{X})\right| \leq C_{d} s|\mathcal{X}|^{d}$ for some universal constant $C_{d} \in(0, \infty)$. Together with Hölder's inequality and the above probability bounds, this yields the asserted expectation and variance bounds.

The results of [34] show that for $\xi_{s} \in\left\{\xi_{0}, \ldots, \xi_{d-1}, \xi_{d, s}\right\}$ one has

$$
\operatorname{Var} S_{\xi_{s}}\left(\mathcal{P}_{s}\right)=\Theta\left(s^{\frac{d-1}{d+1}}\right) \quad \text { and } \quad \operatorname{Var} S_{\xi_{n}}\left(\mathcal{X}_{n}\right)=\Theta\left(n^{\frac{d-1}{d+1}}\right)
$$

For $\xi_{s} \in\left\{\xi_{1, s}, \ldots, \xi_{d-1, s}\right\}$ and taking into account scaling (3.20), we know from Corollary 7.1 of [9] and from Theorem 2 of [3] that

$$
\operatorname{Var} S_{\xi_{s}}\left(\mathcal{P}_{s}\right)=\Theta\left(s^{\frac{d-1}{d+1}}\right) \quad \text { and } \quad \operatorname{Var} S_{\xi_{n}}\left(\mathcal{X}_{n}\right)=\Theta\left(n^{\frac{d-1}{d+1}}\right)
$$

Hence, Lemma 3.9 implies that for $\xi_{s} \in\left\{\xi_{0}, \ldots, \xi_{d-1}, \xi_{1, s}, \ldots, \xi_{d, s}\right\}$

$$
\operatorname{Var} S_{\tilde{\xi}_{s}}\left(\mathcal{P}_{s}\right)=\Theta\left(s^{\frac{d-1}{d+1}}\right) \quad \text { and } \quad \operatorname{Var} S_{\tilde{\xi}_{n}}\left(\mathcal{X}_{n}\right)=\Theta\left(n^{\frac{d-1}{d+1}}\right)
$$

For a point $x \in A$, let $\tilde{H}_{x, 1}, \ldots, \tilde{H}_{x, 2^{d-1}}$ be a decomposition of $H_{x}$ into solid orthants having $x$ in common and let $H_{x, i}:=\tilde{H}_{x, i}+\operatorname{Span}(x)$ for $i \in\left\{1, \ldots, 2^{d-1}\right\}$.

LEMMA 3.10. Let $\tilde{\xi}_{s} \in\left\{\tilde{\xi}_{0}, \ldots, \tilde{\xi}_{d-1}, \tilde{\xi}_{1, s}, \ldots, \tilde{\xi}_{d, s}\right\}$ and let $x \in A, r>0$ and $\mathcal{X} \in \mathbf{N}$ be such that $x \in \mathcal{X}$ and $\mathcal{X} \cap A_{-\rho_{0}} \cap A_{x, r} \cap H_{x, i} \neq \varnothing$ for $i \in\left\{1, \ldots, 2^{d-1}\right\}$. Then, $\tilde{\xi}_{s}(x, \mathcal{X})$ is completely determined by $\mathcal{X} \cap A_{-\rho_{0}} \cap A_{x, r}$, that is to say by $\mathcal{X} \cap A_{-\rho_{0}} \cap B_{\mathrm{d}_{\max }}\left(x, c_{\max } r\right)$ with $c_{\max }$ as in Lemma 3.7. 
Proof. Let $\mathrm{d}\left(x, A^{c}\right) \leq \rho_{0} \leq 1 /\left(8 \bar{c}^{2}\right)$ since, otherwise, the assertion is trivial. By assumption, there are $y_{1}, \ldots, y_{2^{d-1}}$ such that $y_{i} \in \mathcal{X} \cap A_{-\rho_{0}} \cap A_{x, r} \cap H_{x, i}$ for $i \in\left\{1, \ldots, 2^{d-1}\right\}$. Let $C_{x, y_{1}, \ldots, y_{2 d-1}}$ be the cone with apex $x$ generated by the points $\mathbf{0}, y_{1}, \ldots, y_{2^{d-1}}$. If $C_{x, y_{1}, \ldots, y_{2 d-1}}=\mathbb{R}^{d}$, we have $x \in \operatorname{Conv}\left(\left\{\mathbf{0}, y_{1}, \ldots, y_{2^{d-1}}\right\}\right)$, whence $\tilde{\xi}_{s}(x, \mathcal{X})=0$. If $C_{x, y_{1}, \ldots, y_{2} d-1} \neq \mathbb{R}^{d}$ [this implies that $r>\sqrt{\mathrm{d}\left(x, A^{c}\right)}$ ], no point in the interior of $C_{x, y_{1}, \ldots, y_{2} d-1}$ can be connected with $x$ by an edge. Since $\operatorname{Conv}\left(\left(A \backslash B^{d}(x, r / \underline{c})\right) \cup\{x\}\right) \subset C_{x, y_{1}, \ldots, y_{2} d-1}$, all points in $A \backslash A_{x, r}$ are irrelevant for the facial structure at $x$. Consequently, the scores $\tilde{\xi}_{s}$ are completely determined by $\mathcal{X} \cap A_{-\rho_{0}} \cap A_{x, r}$. In view of Lemma 3.7(b), we have $A_{x, r} \subset B_{\mathrm{d}_{\max }}\left(x, c_{\max } r\right)$ so the same is true for $\mathcal{X} \cap A_{-\rho_{0}} \cap B_{\mathrm{d}_{\max }}\left(x, c_{\max } r\right)$.

We define the map $R: A \times \mathbf{N} \rightarrow \mathbb{R}$ which sends $(x, \mathcal{X})$ to $R(x, \mathcal{X} \cup\{x\})$

$$
:=\left\{\begin{array}{l}
c_{\max } \inf \left\{r \geq 0: \mathcal{X} \cap A_{-\rho_{0}} \cap A_{x, r} \cap H_{x, i} \neq \varnothing \text { for } i \in\left\{1, \ldots, 2^{d-1}\right\}\right\}, \\
\quad x \in A_{-\rho_{0}}, \\
0, \quad x \notin A_{-\rho_{0}} .
\end{array}\right.
$$

The next lemma shows that all $\tilde{\xi}_{s} \in\left\{\tilde{\xi}_{0}, \ldots, \tilde{\xi}_{d-1}, \tilde{\xi}_{1, s}, \ldots, \tilde{\xi}_{d, s}\right\}$ satisfy (2.4) and (2.5) with $\alpha_{\text {stab }}=d+1$.

LEMMA 3.11. $R$ is a radius of stabilization for any $\tilde{\xi}_{s} \in\left\{\tilde{\xi}_{0}, \ldots, \tilde{\xi}_{d-1}, \tilde{\xi}_{1, s}\right.$, $\left.\ldots, \tilde{\xi}_{d, s}\right\}$ and there are constants $C, c \in(0, \infty)$ such that for $r \geq 0, x \in A$

$$
\mathbb{P}\left(R\left(x, \mathcal{P}_{s} \cup\{x\}\right) \geq r\right) \leq C \exp \left(-c s r^{d+1}\right), \quad s \geq 1,
$$

whereas

$$
\mathbb{P}\left(R\left(x, \mathcal{X}_{n-8} \cup\{x\}\right) \geq r\right) \leq C \exp \left(-c n r^{d+1}\right), \quad n \geq 9
$$

PROOF. It follows from Lemma 3.10 that $R$ is a radius of stabilization. It is sufficient to establish the desired inequalities for $x \in A_{-\rho_{0}}$ and $r \in\left[0, r_{0}\right]$ for some $r_{0}>0$. We see that

$\mathbb{P}\left(R\left(x, \mathcal{P}_{s} \cup\{x\}\right) \geq r\right) \leq \mathbb{P}\left(\exists i \in\left\{1, \ldots, 2^{d-1}\right\}: \mathcal{P}_{s} \cap A_{-\rho_{0}} \cap A_{x, r / c_{\max }} \cap H_{x, i}=\varnothing\right)$.

Choosing $r_{0}$ small enough so that $A_{x, r / c_{\max }} \cap A_{-\rho_{0}}=A_{x, r / c_{\max }}$ and noting that by the same arguments as in the proof of Lemma 3.7(a) $\mathbb{Q}\left(A_{x, r / c_{\max }} \cap H_{i}\right) \geq$ $c_{\mathbb{Q}} r^{d+1} /\left(2^{d-1} c_{\max }^{d+1}\right)$, we obtain that

$$
\mathbb{P}\left(R\left(x, \mathcal{P}_{s} \cup\{x\}\right) \geq r\right) \leq 2^{d-1} \exp \left(-s c_{\mathbb{Q}} r^{d+1} /\left(2^{d-1} c_{\max }^{d+1}\right)\right) .
$$

The proof for the binomial case goes similarly.

The next lemma shows that all $\tilde{\xi}_{s} \in\left\{\tilde{\xi}_{0}, \ldots, \tilde{\xi}_{d-1}, \tilde{\xi}_{1, s}, \ldots, \tilde{\xi}_{d, s}\right\}$ satisfy (2.8) and (2.9) with $\alpha_{\partial A}=d+1$. 
LeMma 3.12. For any $\tilde{\xi}_{s} \in\left\{\tilde{\xi}_{0}, \ldots, \tilde{\xi}_{d-1}, \tilde{\xi}_{1, s}, \ldots, \tilde{\xi}_{d, s}\right\}$, there are constants $C_{b}, c_{b} \in(0, \infty)$ such that for $x \in A, \mathcal{A} \subset A$ with $|\mathcal{A}| \leq 7$,

$$
\mathbb{P}\left(\tilde{\xi}_{s}\left(x, \mathcal{P}_{s} \cup\{x\} \cup \mathcal{A}\right) \neq 0\right) \leq C_{b} \exp \left(-c_{b} s \mathrm{~d}_{\max }\left(x, A^{c}\right)^{d+1}\right), \quad s \geq 1,
$$

whereas

$$
\mathbb{P}\left(\tilde{\xi}_{n}\left(x, \mathcal{X}_{n-8} \cup\{x\} \cup A\right) \neq 0\right) \leq C_{b} \exp \left(-c_{b} n \mathrm{~d}_{\max }\left(x, A^{c}\right)^{d+1}\right), \quad n \geq 9 .
$$

Proof. For $x \in A_{-\rho_{0}}, \mathcal{X} \in \mathbf{N}$ and $\mathcal{A} \subset A$ with $|\mathcal{A}| \leq 7$, we have that $\tilde{\xi}_{s}(x, \mathcal{X} \cup\{x\} \cup \mathcal{A})=0$ if $R(x, \mathcal{X} \cup\{x\}) \leq \sqrt{\mathrm{d}\left(x, A^{c}\right)}=\mathrm{d}_{\max }\left(x, A^{c}\right)$. Thus the assertions follow from Lemma 3.11.

LEMmA 3.13. For any $q \geq 1$ and $\tilde{\xi}_{s} \in\left\{\tilde{\xi}_{0}, \ldots, \tilde{\xi}_{d-1}, \tilde{\xi}_{1, s}, \ldots, \tilde{\xi}_{d, s}\right\}$, there is a constant $C_{q} \in(0, \infty)$ such that for all $\mathcal{A} \subset A$ with $|\mathcal{A}| \leq 7$,

$$
\sup _{s \geq 1} \sup _{x \in A} \mathbb{E}\left|\tilde{\xi}_{s}\left(x, \mathcal{P}_{s} \cup\{x\} \cup \mathcal{A}\right)\right|^{q} \leq C_{q}
$$

and

$$
\sup _{n \in \mathbb{N}, n \geq 9} \sup _{x \in A} \mathbb{E}\left|\tilde{\xi}_{n}\left(x, \mathcal{X}_{n-8} \cup\{x\} \cup \mathcal{A}\right)\right|^{q} \leq C_{q}
$$

PRoof. The assertion for $\tilde{\xi}_{0}, \ldots, \tilde{\xi}_{d-1}$ can be shown similarly as in Lemma 7.1 of [9]. Similar considerations as in the proof of Lemma 3.10 show that

$$
\tilde{\xi}_{d, s}\left(x, \mathcal{P}_{s} \cup\{x\} \cup \mathcal{A}\right) \leq s \mathbb{Q}\left(A_{x, R\left(x, \mathcal{P}_{s} \cup\{x\}\right)}\right) .
$$

Combining this with Lemma 3.7 and Lemma 3.11 leads to the inequality for $\tilde{\xi}_{d, s}$ in the Poisson case, which can be proven similarly in the binomial case. For the intrinsic volumes $\tilde{\xi}_{j, s}, j \in\{0, \ldots, d-1\}$, the bound (3.21) shows that the $q$ th moment of $\tilde{\xi}_{j, s}$ is bounded by a constant multiple of the $q$ th moment of $\tilde{\xi}_{d, s}$ plus $s^{q} \mathbb{P}\left(B\left(\mathbf{0}, \rho_{0}\right) \not \subset \operatorname{Conv}\left(\mathcal{X}_{s}\right)\right)$, which by Lemma 3.9 is bounded by $s^{q} C_{0} \exp \left(-c_{0} s\right)$. This completes the proof.

LEMma 3.14. For any $\tilde{\xi}_{s} \in\left\{\tilde{\xi}_{0}, \ldots, \tilde{\xi}_{d-1}, \tilde{\xi}_{1, s}, \ldots, \tilde{\xi}_{d, s}\right\}$ there is a constant $\tilde{C} \in(0, \infty)$ such that

$$
d_{K}\left(\frac{S_{\tilde{\xi}_{s}}\left(\mathcal{P}_{s}\right)-\mathbb{E} S_{\tilde{\xi}_{s}}\left(\mathcal{P}_{s}\right)}{\sqrt{\operatorname{Var} S_{\tilde{\xi}_{s}}\left(\mathcal{P}_{s}\right)}}, N\right) \leq \tilde{C} s^{-\frac{d-1}{2(d+1)}}, \quad s \geq 1,
$$

and

$$
d_{K}\left(\frac{S_{\tilde{\xi}_{n}}\left(\mathcal{X}_{n}\right)-\mathbb{E} S_{\tilde{\xi}_{n}}\left(\mathcal{X}_{n}\right)}{\sqrt{\operatorname{Var} S_{\tilde{\xi}_{n}}\left(\mathcal{X}_{n}\right)}}, N\right) \leq \tilde{C} n^{-\frac{d-1}{2(d+1)}}, \quad n \geq 9
$$


ProOF. By Lemmas 3.6, 3.11, 3.12 and 3.13 all conditions of Corollary 2.2 in connection with Remark (vii) after Theorem 2.3 are satisfied with $\mathbb{X}:=A, \tilde{\mathbb{X}}:=$ $A_{-\rho_{0}}$ and $K:=\partial A$. Note that $I_{\partial A, s}=O\left(s^{(d-1) /(d+1)}\right)$, which completes the proof.

Proof of TheOREM 3.5. For any pair $(X, \tilde{X})$ of square integrable random variables satisfying $\operatorname{Var} X, \operatorname{Var} \tilde{X}>0$, a straightforward computation shows that

$$
\begin{aligned}
& d_{K}\left(\frac{X-\mathbb{E} X}{\sqrt{\operatorname{Var} X}}, N\right) \\
& \quad \leq d_{K}\left(\frac{\tilde{X}-\mathbb{E} X}{\sqrt{\operatorname{Var} X}}, N\right)+\mathbb{P}(X \neq \tilde{X}) \\
& \quad=d_{K}\left(\frac{\tilde{X}-\mathbb{E} \tilde{X}}{\sqrt{\operatorname{Var} \tilde{X}}}, N\left(\frac{\mathbb{E} X-\mathbb{E} \tilde{X}}{\sqrt{\operatorname{Var} \tilde{X}}}, \frac{\operatorname{Var} X}{\operatorname{Var} \tilde{X}}\right)\right)+\mathbb{P}(X \neq \tilde{X}) \\
& \quad \leq d_{K}\left(\frac{\tilde{X}-\mathbb{E} \tilde{X}}{\sqrt{\operatorname{Var} \tilde{X}}}, N\right)+d_{K}\left(N, N\left(\frac{\mathbb{E} X-\mathbb{E} \tilde{X}}{\sqrt{\operatorname{Var} \tilde{X}}}, \frac{\operatorname{Var} X}{\operatorname{Var} \tilde{X}}\right)\right)+\mathbb{P}(X \neq \tilde{X}) \\
& \quad \leq d_{K}\left(\frac{\tilde{X}-\mathbb{E} \tilde{X}}{\sqrt{\operatorname{Var} \tilde{X}}}, N\right)+\frac{|\mathbb{E} X-\mathbb{E} \tilde{X}|}{\sqrt{\operatorname{Var} \tilde{X}}+C\left|\frac{\operatorname{Var} X}{\operatorname{Var} \tilde{X}}-1\right|+\mathbb{P}(X \neq \tilde{X}),}
\end{aligned}
$$

where $N\left(\mu, \sigma^{2}\right)$ stands for a Gaussian random variable with mean $\mu$ and variance $\sigma^{2}$ and $C \in(0, \infty)$ is some universal constant. Applying this to the pairs $(X, \tilde{X}):=$ $\left(S_{\xi_{s}}\left(\mathcal{P}_{S}\right), S_{\tilde{\xi}_{s}}\left(\mathcal{P}_{s}\right)\right)$ and $(X, \tilde{X}):=\left(S_{\xi_{n}}\left(\mathcal{X}_{n}\right), S_{\tilde{\xi}_{n}}\left(\mathcal{X}_{n}\right)\right)$, respectively, together with Lemma 3.9, Lemma 3.14, (3.22), (3.23) and (3.24) completes the proof.

3.5. Clique counts in generalized random geometric graphs. Let $(\mathbb{X}, \mathcal{F}, \mathbb{Q})$ be equipped with a semi-metric d such that (2.1) is satisfied for some $\gamma$ and $\kappa$. Moreover, let $\mathbb{M}=[0, \infty)$ be equipped with the Borel sigma algebra $\mathcal{F}_{\mathbb{M}}:=\mathcal{B}([0, \infty))$ and a probability measure $\mathbb{Q}_{\mathbb{M}}$ on $([0, \infty), \mathcal{B}([0, \infty)))$. By $\widehat{\mathbb{Q}}$, we denote the the product measure of $\mathbb{Q}$ and $\mathbb{Q}_{\mathbb{M}}$. In the following, let $\mathcal{P}_{s}$ be a marked Poisson point process with intensity measure $s \widehat{\mathbb{Q}}, s \geq 1$, and let $\mathcal{X}_{n}$ be a marked binomial point process of $n \in \mathbb{N}$ points distributed according to $\widehat{\mathbb{Q}}$.

Given $\mathcal{X} \in \mathbf{N}$, recall that $\mathbf{N}$ is the set of point configurations in $\widehat{\mathbb{X}}$, and a scale parameter $\beta \in(0, \infty)$, consider the graph $G(\mathcal{X}, \beta)$ on $\mathcal{X}$ with $\left(x_{1}, m_{x_{1}}\right) \in \mathcal{X}$ and $\left(x_{2}, m_{x_{2}}\right) \in \mathcal{X}$ joined with an edge iff $\mathrm{d}\left(x_{1}, x_{2}\right) \leq \beta \min \left(m_{x_{1}}, m_{x_{2}}\right)$. When $m_{x}=1$ for all $x \in \mathcal{X}$, we obtain the familiar geometric graph with parameter $\beta$. Alternatively, we could use the connection rule that $\left(x_{1}, m_{x_{1}}\right)$ and $\left(x_{2}, m_{x_{2}}\right)$ are joined with an edge iff $\mathrm{d}\left(x_{1}, x_{2}\right) \leq \beta \max \left(m_{x_{1}}, m_{x_{2}}\right)$. A scale-free random graph based on this connection rule with an underlying marked Poisson point process is studied in [17]. The number of cliques of order $k+1$ in $G(\mathcal{X}, \beta)$, here denoted $\mathcal{C}_{k}(\mathcal{X}, \beta)$, is a well-studied statistic in random geometric graphs. Recall that $k+1$ vertices of a graph form a clique of order $k+1$ if each pair of them is connected by an edge. 
The clique count $\mathcal{C}_{k}(\mathcal{X}, \beta)$ is also a central statistic in topological data analysis. Consider the simplicial complex $\mathcal{R}^{\beta}(\mathcal{X})$ whose $k$-simplices correspond to unordered $(k+1)$-tuples of points of $\mathcal{X}$ such that any constituent pair of points $\left(x_{1}, m_{x_{1}}\right)$ and $\left(x_{2}, m_{x_{2}}\right)$ satisfies $\mathrm{d}\left(x_{1}, x_{2}\right) \leq \beta \min \left(m_{x_{1}}, m_{x_{2}}\right)$. When $m_{x}=1$ for all $x \in \mathcal{X}$, then $\mathcal{R}^{\beta}(\mathcal{X})$ coincides with the Vietoris-Rips complex with scale parameter $\beta$ and $\mathcal{C}_{k}(\mathcal{X}, \beta)$ counts the number of $k$-simplices in $\mathcal{R}^{\beta}(\mathcal{X})$.

When $\mathbb{Q}$ is the uniform measure on a compact set $\mathbb{X} \subset \mathbb{R}^{d}$ with $\operatorname{Vol}(\mathbb{X})>0$ and $\gamma=d$, the ungainly quantity $\mathcal{C}_{k}\left(\mathcal{P}_{s}, \beta s^{-1 / \gamma}\right)$ studied below is equivalent to the more natural clique count $\mathcal{C}_{k}\left(\widetilde{\mathcal{P}}_{1} \cap s^{1 / d} \mathbb{X}, \beta\right)$, where $\widetilde{\mathcal{P}}_{1}$ is a rate one stationary Poisson point process in $\mathbb{R}^{d}$ and $\widetilde{\mathcal{P}}_{1} \cap s^{1 / d} \mathbb{X}$ is its restriction to $s^{1 / d} \mathbb{X}$.

THEOREM 3.15. Let $k \in \mathbb{N}$ and $\beta \in(0, \infty)$ and assume there are constants $c_{1} \in(0, \infty)$ and $c_{2} \in(0, \infty)$ such that

$$
\mathbb{P}\left(M_{x} \geq r\right) \leq c_{1} \exp \left(-\frac{r^{c_{2}}}{c_{1}}\right), \quad x \in \mathbb{X}, r \in(0, \infty) .
$$

If $\inf _{s \geq 1} \operatorname{Var} \mathcal{C}_{k}\left(\mathcal{P}_{s}, \beta s^{-1 / \gamma}\right) / s>0$, then there is a constant $\tilde{C} \in(0, \infty)$ such that

$$
d_{K}\left(\frac{\mathcal{C}_{k}\left(\mathcal{P}_{s}, \beta s^{-1 / \gamma}\right)-\mathbb{E} \mathcal{C}_{k}\left(\mathcal{P}_{s}, \beta s^{-1 / \gamma}\right)}{\sqrt{\operatorname{Var} \mathcal{C}_{k}\left(\mathcal{P}_{s}, \beta s^{-1 / \gamma}\right)}}, N\right) \leq \frac{\tilde{C}}{\sqrt{s}}, \quad s \geq 1
$$

Likewise if $\inf _{n \geq 9} \operatorname{Var}_{k}\left(\mathcal{X}_{n}, \beta n^{-1 / \gamma}\right) / n>0$, then there is a constant $\tilde{C} \in(0, \infty)$ such that

$$
d_{K}\left(\frac{\mathcal{C}_{k}\left(\mathcal{X}_{n}, \beta n^{-1 / \gamma}\right)-\mathbb{E}_{k}\left(\mathcal{X}_{n}, \beta n^{-1 / \gamma}\right)}{\sqrt{\operatorname{Var} \mathcal{C}_{k}\left(\mathcal{X}_{n}, \beta n^{-1 / \gamma}\right)}}, N\right) \leq \frac{\tilde{C}}{\sqrt{n}}, \quad n \geq 9
$$

REMARKS. (i) When $\mathbb{X}$ is a full-dimensional subset of $\mathbb{R}^{d}$ and when $M_{x} \equiv 1$ for all $x \in \mathbb{X}$, that is, $\mathbb{Q}_{\mathbb{M}}$ is the Dirac measure concentrated at one, a central limit theorem for the Poisson case is shown in [24], Theorem 3.10. Although the result in [24] is nonquantitative, the method of proof should yield a rate of convergence for the Kolmogorov distance. Rates of normal convergence with respect to the Wasserstein distance $d_{W}$ are given in [12].

(ii) The contributions of this theorem are three-fold. First, $\mathbb{X}$ may be an arbitrary metric space, not necessarily a subset of $\mathbb{R}^{d}$. Second, the graphs $G\left(\mathcal{P}_{s}, \beta s^{-1 / \gamma}\right)$ and $G\left(\mathcal{X}_{n}, \beta n^{-1 / \gamma}\right)$ are more general than the standard random geometric graph, as they consist of edges having arbitrary (exponentially decaying) lengths. Third, by applying our general findings we obtain presumably optimal rates of convergence for the Poisson and the binomial case at the same time.

(iii) The random variable $\mathcal{C}_{k}\left(\mathcal{P}_{s}, \beta s^{-1 / \gamma}\right)$ is a so-called Poisson U-statistic. Bounds for the normal approximation of such random variables were deduced, for example, in [35] and [20] for the Wasserstein distance and in [43] and [14] for 
the Kolmogorov distance. These results should also yield bounds similar to those in (3.26).

(iv) The assumption $\inf _{s \geq 1} \operatorname{Var} \mathcal{C}_{k}\left(\mathcal{P}_{s}, \beta s^{-1 / \gamma}\right) / s>0$ is satisfied if $\mathbb{X} \subset \mathbb{R}^{d}$ is a full $d$-dimensional set and $g$ is a bounded probability density, as noted in the proof of Theorem 2.5 in Section 6 of [31]. If this assumption is not satisfied, then we would have instead

$$
\begin{aligned}
d_{K}( & \left.\frac{\mathcal{C}_{k}\left(\mathcal{P}_{s}, \beta s^{-1 / \gamma}\right)-\mathbb{E} \mathcal{C}_{k}\left(\mathcal{P}_{s}, \beta s^{-1 / \gamma}\right)}{\sqrt{\operatorname{Var} \mathcal{C}_{k}\left(\mathcal{P}_{s}, \beta s^{-1 / \gamma}\right)}}, N\right) \\
\leq & \tilde{C}\left(\frac{\sqrt{s}}{\operatorname{Var} \mathcal{C}_{k}\left(\mathcal{P}_{s}, \beta s^{-1 / \gamma}\right)}\right. \\
& \left.+\frac{s}{\left(\operatorname{Var} \mathcal{C}_{k}\left(\mathcal{P}_{s}, \beta s^{-1 / \gamma}\right)\right)^{3 / 2}}+\frac{s^{3 / 2}}{\left(\operatorname{Var} \mathcal{C}_{k}\left(\mathcal{P}_{s}, \beta s^{-1 / \gamma}\right)\right)^{2}}\right)
\end{aligned}
$$

for $s \geq 1$. A similar comment applies for an underlying binomial point process in the situation where $\inf _{n \geq 9} \operatorname{Var}_{k}\left(\mathcal{X}_{n}, \beta n^{-1 / \gamma}\right) / n>0$ does not hold.

Proof of TheOrem 3.15. To deduce Theorem 3.15 from Corollary 2.2, we express $\mathcal{C}_{k}\left(\mathcal{X}, \beta s^{-1 / \gamma}\right)$ as a sum of stabilizing score functions, which goes as follows. Fix $\gamma, s, \beta \in(0, \infty)$. For $\mathcal{X} \in \mathbf{N}$ and $x \in \mathcal{X}$, let $\phi_{k, s}^{(\beta)}(x, \mathcal{X})$ be the number of $(k+1)$-cliques containing $x$ in $G\left(\mathcal{X}, \beta s^{-1 / \gamma}\right)$ and such that $x$ is the point with the largest mark. This gives the desired identification

$$
\mathcal{C}_{k}\left(\mathcal{X}, \beta s^{-1 / \gamma}\right)=\sum_{x \in \mathcal{X}} \phi_{k, s}^{(\beta)}(x, \mathcal{X})
$$

Now we are ready to deduce (3.26) and (3.27) from Corollary 2.2 with the scores $\xi_{s}$ and $\xi_{n}$ set to $\phi_{k, s}^{(\beta)}$ and $\phi_{k, n}^{(\beta)}$, respectively, and with $K$ set to $\mathbb{X}$. Notice that $I_{K, s}=\Theta(s)$, as noted in (2.13). It is enough to show that $\phi_{k, s}^{(\beta)}$ and $\phi_{k, n}^{(\beta)}$ satisfy all conditions of Corollary 2.2. Stabilization (2.4) is satisfied with $\alpha_{\mathrm{stab}}=a$, with the radius of stabilization

$$
R_{s}\left(\left(x, M_{x}\right), \mathcal{P}_{s} \cup\left\{\left(x, M_{x}\right)\right\}\right)=\beta s^{-1 / \gamma} M_{x},
$$

because $M_{x}$ has exponentially decaying tails as in (3.25). For any $p>0$, we have

$$
\begin{aligned}
& \mathbb{E}\left|\phi_{k, s}^{(\beta)}\left(\left(x, M_{x}\right), \mathcal{P}_{s} \cup\left\{\left(x, M_{x}\right) \cup\left(\mathcal{A}, M_{\mathcal{A}}\right)\right\}\right)\right|^{4+p} \\
& \quad \leq \mathbb{E}\left|\operatorname{card}\left\{\mathcal{P}_{s} \cap B\left(x, \beta s^{-1 / \gamma} M_{x}\right)\right\}+7\right|^{(4+p) k} \leq C(\beta, p, \gamma)<\infty
\end{aligned}
$$

for all $x \in \mathbb{X}, s \geq 1$ and $\mathcal{A} \subset \mathbb{X}$ with $|\mathcal{A}| \leq 7$ and so the $(4+p)$ th moment condition (2.6) holds for $p \in(0, \infty)$. The conclusion (3.26) follows from (2.14). The proof of (3.27) is similar. 
4. Malliavin-Stein bounds. For any measurable $f: \mathbf{N} \rightarrow \mathbb{R}, \mathcal{M} \in \mathbf{N}$ and $\hat{x}, \hat{x}_{1}, \hat{x}_{2} \in \widehat{\mathbb{X}}$, we define

$$
\begin{aligned}
D_{\hat{x}} f(\mathcal{M}) & :=f(\mathcal{M} \cup\{\hat{x}\})-f(\mathcal{M}), \\
D_{\hat{x}_{1}, \hat{x}_{2}}^{2} f(\mathcal{M}) & :=f\left(\mathcal{M} \cup\left\{\hat{x}_{1}, \hat{x}_{2}\right\}\right)-f\left(\mathcal{M} \cup\left\{\hat{x}_{1}\right\}\right)-f\left(\mathcal{M} \cup\left\{\hat{x}_{2}\right\}\right)+f(\mathcal{M}) .
\end{aligned}
$$

Our key tool for the proof of the bound (2.11) is the following marked version of a result from [22] (see Proposition 1.4 and Theorem 6.1 in [22]) for square integrable Poisson functionals.

THEOREM 4.1. Let $s>0$ and let $f: \mathbf{N} \rightarrow \mathbb{R}$ be measurable with $\mathbb{E} f\left(\mathcal{P}_{s}\right)^{2}<$ $\infty$. Assume there are constants $c, p \in(0, \infty)$ such that

$$
\mathbb{E}\left|D_{\left(x, M_{x}\right)} f\left(\mathcal{P}_{s} \cup\left\{\left(\mathcal{A}, M_{\mathcal{A}}\right)\right\}\right)\right|^{4+p} \leq c, \quad \mathbb{Q} \text {-a.e. } x \in \mathbb{X}, \mathcal{A} \subset \mathbb{X},|\mathcal{A}| \leq 1 .
$$

Let $F:=f\left(\mathcal{P}_{S}\right)$. Then there is a constant $C:=C(c, p) \in(0, \infty)$ such that

$$
d_{K}\left(\frac{F-\mathbb{E} F}{\sqrt{\operatorname{Var} F}}, N\right) \leq C\left(S_{1}+S_{2}+S_{3}\right),
$$

with

$$
\begin{aligned}
\Gamma_{s} & :=s \int_{\mathbb{X}} \mathbb{P}\left(D_{\left(x, M_{x}\right)} f\left(\mathcal{P}_{s}\right) \neq 0\right)^{\frac{p}{8+2 p}} \mathbb{Q}(\mathrm{d} x), \\
\psi_{s}\left(x_{1}, x_{2}\right) & :=\mathbb{P}\left(D_{\left(x_{1}, M_{x_{1}}\right),\left(x_{2}, M_{x_{2}}\right)}^{2} f\left(\mathcal{P}_{s}\right) \neq 0\right)^{\frac{p}{16+4 p}} \\
S_{1} & :=\frac{s}{\operatorname{Var} F} \sqrt{\int_{\mathbb{X}^{2}} \psi_{s}\left(x_{1}, x_{2}\right)^{2} \mathbb{Q}^{2}\left(\mathrm{~d}\left(x_{1}, x_{2}\right)\right)} \\
S_{2} & :=\frac{s^{3 / 2}}{\operatorname{Var} F} \sqrt{\int_{\mathbb{X}}\left(\int_{\mathbb{X}} \psi_{s}\left(x_{1}, x_{2}\right) \mathbb{Q}\left(\mathrm{d} x_{2}\right)\right)^{2} \mathbb{Q}\left(\mathrm{d} x_{1}\right)}, \\
S_{3} & :=\frac{\sqrt{\Gamma_{s}}}{\operatorname{Var} F}+\frac{2 \Gamma_{s}}{(\operatorname{Var} F)^{3 / 2}}+\frac{\Gamma_{s}^{5 / 4}+2 \Gamma_{s}^{3 / 2}}{(\operatorname{Var} F)^{2}}
\end{aligned}
$$

PROOF. In case that there are no marks, this is Theorem 6.1 in [22]. The marked version can be obtained in the following way: In Theorem 1.2 in [22], one can use the product form of $\widehat{\mathbb{Q}}$ and Hölder's inequality to bring the marks under the expectations. Evaluating this new bound along the lines of the proof of Theorem 6.1 in [22] yields (4.2).

For the case of binomial input, we do not have the same ready-made bounds at our disposal. We fill this lacuna with the following analogous bound, bringing the results from [20] and [22] into a satisfying alignment. 
THEOREM 4.2. Let $n \geq 3$ and let $f: \mathbf{N} \rightarrow \mathbb{R}$ be measurable with $\mathbb{E} f\left(\mathcal{X}_{n}\right)^{2}<$ $\infty$. Assume that there are constants $c, p \in(0, \infty)$ such that

(4.3)

$\mathbb{E}\left|D_{\left(x, M_{x}\right)} f\left(\mathcal{X}_{n-1-|\mathcal{A}|} \cup\left\{\left(\mathcal{A}, M_{\mathcal{A}}\right)\right\}\right)\right|^{4+p} \leq c, \quad \mathbb{Q}$-a.e. $x \in \mathbb{X}, \mathcal{A} \subset \mathbb{X},|\mathcal{A}| \leq 2$.

Let $F:=f\left(\mathcal{X}_{n}\right)$. Then there is a constant $C:=C(c, p) \in(0, \infty)$ such that

$$
d_{K}\left(\frac{F-\mathbb{E} F}{\sqrt{\operatorname{Var} F}}, N\right) \leq C\left(S_{1}^{\prime}+S_{2}^{\prime}+S_{3}^{\prime}\right),
$$

with

$$
\begin{aligned}
\Gamma_{n}^{\prime} & :=n \int_{\mathbb{X}} \mathbb{P}\left(D_{\left(x, M_{x}\right)} f\left(\mathcal{X}_{n-1}\right) \neq 0\right)^{\frac{p}{8+2 p}} \mathbb{Q}(\mathrm{d} x) \\
\psi_{n}^{\prime}\left(x, x^{\prime}\right) & :=\sup _{\mathcal{A} \subset \mathbb{X}:|\mathcal{A}| \leq 1} \mathbb{P}\left(D_{\left(x, M_{x}\right),\left(x^{\prime}, M_{x^{\prime}}\right)}^{2} f\left(\mathcal{X}_{n-2-|\mathcal{A}|} \cup\left(\mathcal{A}, M_{\mathcal{A}}\right)\right) \neq 0\right)^{\frac{p}{8+2 p}} \\
S_{1}^{\prime} & :=\frac{n}{\operatorname{Var} F} \sqrt{\int_{\mathbb{X}^{2}} \psi_{n}^{\prime}\left(x, x^{\prime}\right) \mathbb{Q}^{2}\left(\mathrm{~d}\left(x, x^{\prime}\right)\right)} \\
S_{2}^{\prime} & :=\frac{n^{3 / 2}}{\operatorname{Var} F} \sqrt{\int_{\mathbb{X}}\left(\int_{\mathbb{X}} \psi_{n}^{\prime}\left(x, x^{\prime}\right) \mathbb{Q}\left(\mathrm{d} x^{\prime}\right)\right)^{2} \mathbb{Q}(\mathrm{d} x)} \\
S_{3}^{\prime} & :=\frac{\sqrt{\Gamma_{n}^{\prime}}}{\operatorname{Var} F}+\frac{\Gamma_{n}^{\prime}}{(\operatorname{Var} F)^{3 / 2}}+\frac{{\sqrt{\Gamma_{n}^{\prime}}}^{3}+\Gamma_{n}^{\prime}}{(\operatorname{Var} F)^{2}}
\end{aligned}
$$

Before proving Theorem 4.2, we require two auxiliary results, the first of which involves some additional notation. For a measurable $f: \mathbf{N} \rightarrow \mathbb{R}$ extend the notation $f\left(x_{1}, \ldots, x_{q}\right):=f\left(\left\{x_{1}, \ldots, x_{q}\right\}\right)$ for $x_{1}, \ldots, x_{q} \in \mathbb{X}$.

For a fixed $n \geq 1$, let $X:=\left(X_{1}, \ldots, X_{n}\right)$, where $X_{1}, \ldots, X_{n}$ are independent random elements in $\widehat{\mathbb{X}}$ distributed according to $\widehat{\mathbb{Q}}$. Let $X^{\prime}, \tilde{X}$ be independent copies of $X$. We write $U \stackrel{\text { a.s. }}{=} V$ if two variables $U$ and $V$ satisfy $\mathbb{P}(U=V)=1$. In the vocabulary of [20], a random vector $Y:=\left(Y_{1}, \ldots, Y_{n}\right)$ is a recombination of $\left\{X, X^{\prime}, \tilde{X}\right\}$ if for each $1 \leq i \leq n$, either $Y_{i} \stackrel{\text { a.s. }}{=} X_{i}, Y_{i} \stackrel{\text { a.s. }}{=} X_{i}^{\prime}$ or $Y_{i} \stackrel{\text { a.s. }}{=} \widetilde{X}_{i}$. For a vector $x=\left(x_{1}, \ldots, x_{p}\right) \in \widehat{\mathbb{X}}^{p}$, and indices $I:=\left\{i_{1}, \ldots, i_{q}\right\} \subset[p]:=\{1,2, \ldots, p\}$, define $x^{i_{1}, \ldots, i_{q}}:=\left(x_{j}, j \notin I\right)$, the vector $x$ with the components indexed by $I$ removed. For $i, j \in[n]$, introduce the index derivatives

$$
\begin{aligned}
\mathbf{D}_{i} f(X) & :=f(X)-f\left(X^{i}\right), \\
\mathbf{D}_{i, j}^{2} f(X) & :=f(X)-f\left(X^{i}\right)-f\left(X^{j}\right)+f\left(X^{i, j}\right)=\mathbf{D}_{j, i}^{2} f(X) .
\end{aligned}
$$

We note that the derivatives $D$ and $\mathbf{D}$ obey the relation $\mathbf{D}_{i} f(X)=D_{X_{i}} f\left(\mathcal{X}_{n}^{i}\right)$.

We introduce, for $n$-dimensional random vectors $Y, Y^{\prime}$ and $Z$,

$$
\gamma_{Y, Z}(f):=\mathbb{E}\left[\mathbf{1}_{\left\{\mathbf{D}_{1,2}^{2} f(Y) \neq 0\right\}}\left(\mathbf{D}_{2} f(Z)\right)^{4}\right]
$$




$$
\begin{aligned}
\gamma_{Y, Y^{\prime}, Z}^{\prime}(f) & :=\mathbb{E}\left[\mathbf{1}_{\left\{\mathbf{D}_{1,2}^{2} f(Y) \neq 0, \mathbf{D}_{1,3}^{2} f\left(Y^{\prime}\right) \neq 0\right\}}\left(\mathbf{D}_{2} f(Z)\right)^{4}\right], \\
B_{n}(f) & :=\sup \left\{\gamma_{Y, Z}(f) ; Y, Z \text { recombinations of }\left\{X, X^{\prime}, \widetilde{X}\right\}\right\}, \\
B_{n}^{\prime}(f) & :=\sup \left\{\gamma_{Y, Y^{\prime}, Z}^{\prime}(f) ; Y, Y^{\prime}, Z \text { recombinations of }\left\{X, X^{\prime}, \widetilde{X}\right\}\right\} .
\end{aligned}
$$

Theorem 5.1 of [20], simplified by [20], Remark 5.2, and [20], Proposition 5.3, gives the following.

THEOREM 4.3. Let $n \geq 2, f: \mathbf{N} \rightarrow \mathbb{R}$ measurable with $\mathbb{E} f\left(\mathcal{X}_{n}\right)^{2}<\infty$, and $F:=f\left(\mathcal{X}_{n}\right)$. Then there is a constant $c_{0} \in(0, \infty)$, depending neither on $n$ nor $f$, such that

$$
\begin{aligned}
& d_{K}\left(\frac{F-\mathbb{E} F}{\sqrt{\operatorname{Var} F}}, N\right) \\
& \leq c_{0}\left[\frac{\sqrt{n}}{\operatorname{Var} F}\left(\sqrt{n B_{n}(f)}+\sqrt{n^{2} B_{n}^{\prime}(f)}+\sqrt{\mathbb{E} \mathbf{D}_{1} f(X)^{4}}\right)\right. \\
& \quad+\sup _{Y} \frac{n}{(\operatorname{Var} F)^{2}} \mathbb{E}\left|(f(X)-\mathbb{E} F)\left(\mathbf{D}_{1} f(Y)\right)^{3}\right| \\
& \left.\quad+\frac{n}{(\operatorname{Var} F)^{3 / 2}} \mathbb{E}\left|\mathbf{D}_{1} f(X)\right|^{3}\right],
\end{aligned}
$$

where the $\sup _{Y}$ runs over recombinations $Y$ of $\left\{X, X^{\prime}, \tilde{X}\right\}$.

To control the fourth centered moment of $F:=f\left(\mathcal{X}_{n}\right)$, we use the following bound. For a similar bound for Poisson functionals, we refer to [22], Lemma 4.2.

LEMMA 4.4. For a measurable $f: \mathbf{N} \rightarrow \mathbb{R}, n \in \mathbb{N}$ and $F:=f\left(\mathcal{X}_{n}\right)$ assume that $\operatorname{Var} F=1$. Then

$$
\begin{aligned}
\mathbb{E}(F-\mathbb{E} F)^{4} \leq & 9 \max \left\{\left(32 n \int_{\mathbb{X}} \sqrt{\mathbb{E}\left(D_{\left(x, M_{x}\right)} f\left(\mathcal{X}_{n-1}\right)\right)^{4}} \mathbb{Q}(\mathrm{d} x)\right)^{2},\right. \\
& \left.4 n \mathbb{E}\left(\mathbf{D}_{1} f\left(\mathcal{X}_{n}\right)\right)^{4}+1\right\} .
\end{aligned}
$$

PROOF. The Efron-Stein inequality implies that for measurable $g: \mathbf{N} \rightarrow \mathbb{R}$ and $n \in \mathbb{N}$ such that $\mathbb{E}\left|g\left(\mathcal{X}_{n}\right)\right|<\infty$,

$$
\mathbb{E} g\left(\mathcal{X}_{n}\right)^{2} \leq 2 n \mathbb{E}\left(\mathbf{D}_{1} g\left(\mathcal{X}_{n}\right)\right)^{2}+\left(\mathbb{E} g\left(\mathcal{X}_{n}\right)\right)^{2}
$$

Using the Efron-Stein bound and Var $F=1$ gives

$$
\mathbb{E}(F-\mathbb{E} F)^{4} \leq 2 n \mathbb{E}\left(\mathbf{D}_{1}\left(\left(f\left(\mathcal{X}_{n}\right)-\mathbb{E} F\right)^{2}\right)\right)^{2}+1 .
$$


Combining the identity

$$
\begin{aligned}
\mathbf{D}_{1}\left(g\left(\mathcal{X}_{n}\right)^{2}\right) & =g\left(\mathcal{X}_{n}\right)^{2}-g\left(\mathcal{X}_{n}^{1}\right)^{2}=\left(g\left(\mathcal{X}_{n}^{1}\right)+\mathbf{D}_{1} g\left(\mathcal{X}_{n}\right)\right)^{2}-g\left(\mathcal{X}_{n}^{1}\right)^{2} \\
& =2 g\left(\mathcal{X}_{n}^{1}\right) \mathbf{D}_{1} g\left(\mathcal{X}_{n}\right)+\left(\mathbf{D}_{1} g\left(\mathcal{X}_{n}\right)\right)^{2}
\end{aligned}
$$

with Jensen's inequality, we obtain

$$
\begin{aligned}
\mathbb{E}(F-\mathbb{E} F)^{4} & \leq 2 n \mathbb{E}\left[\left(2 \mathbf{D}_{1} f\left(\mathcal{X}_{n}\right)\left(f\left(\mathcal{X}_{n}^{1}\right)-\mathbb{E} F\right)+\left(\mathbf{D}_{1} f\left(\mathcal{X}_{n}\right)\right)^{2}\right)^{2}\right]+1 \\
& \leq 4 n \mathbb{E}\left[4\left(\mathbf{D}_{1} f\left(\mathcal{X}_{n}\right)\right)^{2}\left(f\left(\mathcal{X}_{n}^{1}\right)-\mathbb{E} F\right)^{2}+\left(\mathbf{D}_{1} f\left(\mathcal{X}_{n}\right)\right)^{4}\right]+1
\end{aligned}
$$

Hölder's inequality and a combination of the triangle inequality and Jensen's inequality imply that

$$
\begin{aligned}
\mathbb{E}\left(\mathbf{D}_{1}\right. & \left.f\left(\mathcal{X}_{n}\right)\right)^{2}\left(f\left(\mathcal{X}_{n}^{1}\right)-\mathbb{E} F\right)^{2} \\
\leq & \int_{\widehat{\mathbb{X}}} \sqrt{\mathbb{E}\left(f\left(\mathcal{X}_{n}^{1} \cup\{y\}\right)-f\left(\mathcal{X}_{n}^{1}\right)\right)^{4}} \widehat{\mathbb{Q}}(\mathrm{d} y) \sqrt{\mathbb{E}\left(f\left(\mathcal{X}_{n}^{1}\right)-\mathbb{E} F\right)^{4}} \\
\leq & \int_{\mathbb{X}} \sqrt{\mathbb{E}\left(D_{\left(x, M_{x}\right)} f\left(\mathcal{X}_{n-1}\right)\right)^{4}} \mathbb{Q}(\mathrm{d} x) 2\left(\sqrt{\mathbb{E}\left(f\left(\mathcal{X}_{n}\right)-\mathbb{E} F\right)^{4}}\right. \\
& +\sqrt{\left.\mathbb{E}\left(\mathbf{D}_{1} f\left(\mathcal{X}_{n}\right)\right)^{4}\right) .}
\end{aligned}
$$

Combining the above estimates, we arrive at

$$
\begin{aligned}
& \mathbb{E}(F-\mathbb{E} F)^{4} \\
& \leq 32 n \int_{\mathbb{X}} \sqrt{\mathbb{E}\left(D_{\left(x, M_{x}\right)} f\left(\mathcal{X}_{n-1}\right)\right)^{4}} \mathbb{Q}(\mathrm{d} x)\left(\sqrt{\mathbb{E}(F-\mathbb{E} F)^{4}}+\sqrt{\mathbb{E}\left(\mathbf{D}_{1} f\left(\mathcal{X}_{n}\right)\right)^{4}}\right) \\
& \quad+4 n \mathbb{E}\left(\mathbf{D}_{1} f\left(\mathcal{X}_{n}\right)\right)^{4}+1,
\end{aligned}
$$

which implies the asserted inequality.

Given Lemma 4.4, we deduce Theorem 4.2 from Theorem 4.3 as follows.

PROOF OF THEOREM 4.2. It suffices to show that each of the five terms in (4.5) is bounded by a scalar multiple of $S_{1}^{\prime}, S_{2}^{\prime}$ or $S_{3}^{\prime}$. We first show that the terms in (4.5) involving $B_{n}(f)$ and $B_{n}^{\prime}(f)$ are bounded, respectively, by scalar multiples of $S_{1}^{\prime}$ and $S_{2}^{\prime}$. Let us estimate first $B_{n}(f)$. By $\widehat{\mathbb{Q}}^{Y_{1}, Y_{2}, Z_{1}, Z_{2}}$ we denote the joint probability measure of $Y_{1}, Y_{2}, Z_{1}, Z_{2}$ and by $\mathbb{Q}^{Y_{1}, Y_{2}, Z_{1}, Z_{2}}$ the joint probability measure of $Y_{1}, Y_{2}, Z_{1}, Z_{2}$ without marks. By Hölder's inequality, the fact that $\widehat{\mathbb{Q}}^{Y_{1}, Y_{2}, Z_{1}, Z_{2}}$ factorizes into $\mathbb{Q}^{Y_{1}, Y_{2}, Z_{1}, Z_{2}}$ and a part controlling the marks, the independence of $Y_{1}, Y_{2}$ and (4.3), we obtain that

$\gamma_{Y, Z}(f)$

$$
=\mathbb{E}\left[\mathbf{1}_{\left\{\mathbf{D}_{1,2}^{2} f(Y) \neq 0\right\}}\left(\mathbf{D}_{2} f(Z)\right)^{4}\right]
$$




$$
\begin{aligned}
= & \int_{\widehat{\mathbb{X}}^{4}} \mathbb{E}\left[\mathbf{1}_{\left\{D_{\hat{y}_{1}, \hat{y}_{2}}^{2} f\left(Y^{1,2}\right) \neq 0\right\}}\left(D_{\hat{z}_{2}} f\left(Z^{1,2} \cup\left\{\hat{z}_{1}\right\}\right)\right)^{4}\right] \\
& \times \widehat{\mathbb{Q}}^{Y_{1}, Y_{2}, Z_{1}, Z_{2}}\left(\mathrm{~d}\left(\hat{y}_{1}, \hat{y}_{2}, \hat{z}_{1}, \hat{z}_{2}\right)\right) \\
\leq & \int_{\widehat{\mathbb{X}}^{4}} \mathbb{P}\left(D_{\hat{y}_{1}, \hat{y}_{2}}^{2} f\left(Y^{1,2}\right) \neq 0\right)^{\frac{p}{4+p}} \mathbb{E}\left[\left|D_{\hat{z}_{2}} f\left(Z^{1,2} \cup\left\{\hat{z}_{1}\right\}\right)\right|^{4+p}\right]^{\frac{4}{4+p}} \\
& \times \widehat{\mathbb{Q}}^{Y_{1}, Y_{2}, Z_{1}, Z_{2}}\left(\mathrm{~d}\left(\hat{y}_{1}, \hat{y}_{2}, \hat{z}_{1}, \hat{z}_{2}\right)\right) \\
\leq & \int_{\mathbb{X}^{4}} \mathbb{P}\left(D_{\left(y_{1}, M_{y_{1}}\right),\left(y_{2}, M_{y_{2}}\right)}^{2} f\left(Y^{1,2}\right) \neq 0\right)^{\frac{p}{4+p}} \\
& \times \mathbb{E}\left[\left|D_{\left(z_{2}, M_{z_{2}}\right)} f\left(Z^{1,2} \cup\left\{\left(z_{1}, M_{z_{1}}\right)\right\}\right)\right|^{4+p}\right]^{\frac{4}{4+p}} \\
& \times \mathbb{Q}^{Y_{1}, Y_{2}, Z_{1}, Z_{2}}\left(\mathrm{~d}\left(y_{1}, y_{2}, z_{1}, z_{2}\right)\right) \\
\leq & c^{\frac{4}{4+p}} \int_{\mathbb{X}^{2}} \mathbb{P}\left(D_{\left(y_{1}, M_{y_{1}}\right),\left(y_{2}, M_{y_{2}}\right)}^{2} f\left(\mathcal{X}_{n-2}\right) \neq 0\right)^{\frac{p}{4+p}} \mathbb{Q}^{2}\left(\mathrm{~d}\left(y_{1}, y_{2}\right)\right) .
\end{aligned}
$$

This implies that

$$
\gamma_{Y, Z}(f) \leq c^{\frac{4}{4+p}} \int_{\mathbb{X}^{2}} \psi_{n}^{\prime}\left(y_{1}, y_{2}\right) \mathbb{Q}^{2}\left(\mathrm{~d}\left(y_{1}, y_{2}\right)\right),
$$

which gives the desired bound

$$
\frac{\sqrt{n}}{\operatorname{Var} F} \sqrt{n B_{n}(f)} \leq c^{\frac{2}{4+p}} \frac{n}{\operatorname{Var} F} \sqrt{\int_{\mathbb{X}^{2}} \psi_{n}^{\prime}\left(x, x^{\prime}\right) \mathbb{Q}^{2}\left(\mathrm{~d}\left(x, x^{\prime}\right)\right)} \leq C(c, p) S_{1}^{\prime} .
$$

To estimate $B_{n}^{\prime}(f)$, let $\widehat{\mathbb{Q}}^{\left(Y_{1}, \ldots, Z_{3}\right)}$ be the joint probability measure of

$$
\left(Y_{1}, \ldots, Y_{3}, Y_{1}^{\prime}, \ldots, Y_{3}^{\prime}, Z_{1}, \ldots, Z_{3}\right)
$$

and let $\mathbb{Q}^{\left(Y_{1}, \ldots, Z_{3}\right)}$ be the corresponding probability measure without marks. By similar arguments as above, we obtain that

$\gamma_{Y, Y^{\prime}, Z}^{\prime}(f)$

$$
\begin{aligned}
= & \mathbb{E}\left[\mathbf{1}_{\left\{\mathbf{D}_{1,2}^{2} f(Y) \neq 0, \mathbf{D}_{1,3}^{2} f\left(Y^{\prime}\right) \neq 0\right\}}\left(\mathbf{D}_{2} f(Z)\right)^{4}\right] \\
= & \int_{\widehat{\mathbb{X}}^{9}} \mathbb{E}\left[\mathbf{1}_{\left\{D_{\hat{y}_{1}, \hat{y}_{2}}^{2} f\left(Y^{1,2,3} \cup\left\{\hat{y}_{3}\right\}\right) \neq 0\right\}} \mathbf{1}_{\left\{D_{\hat{y}_{1}^{\prime}, \hat{y}_{3}^{\prime}}^{2} f\left(Y^{\prime 1,2,3} \cup\left\{\hat{y}_{2}^{\prime}\right\}\right) \neq 0\right\}}\right. \\
& \left.\times\left(D_{\hat{z}_{2}} f\left(Z^{1,2,3} \cup\left\{\hat{z}_{1}, \hat{z}_{3}\right\}\right)\right)^{4}\right] \widehat{\mathbb{Q}}^{Y_{1}, \ldots, Z_{3}}\left(\mathrm{~d}\left(\hat{y}_{1}, \ldots, \hat{z}_{3}\right)\right) \\
\leq & \int_{\widehat{\mathbb{X}}^{9}} \mathbb{P}\left(D_{\hat{y}_{1}, \hat{y}_{2}}^{2} f\left(Y^{1,2,3} \cup\left\{\hat{y}_{3}\right\}\right) \neq 0\right)^{\frac{p}{8+2 p}} \mathbb{P}\left(D_{\hat{y}_{1}^{\prime}, \hat{y}_{3}^{\prime}}^{2} f\left(Y^{\prime 1,2,3} \cup\left\{\hat{y}_{2}^{\prime}\right\}\right) \neq 0\right)^{\frac{p}{8+2 p}} \\
& \times \mathbb{E}\left[\left|D_{\hat{z}_{2}} f\left(Z^{1,2,3} \cup\left\{\hat{z}_{1}, \hat{z}_{3}\right\}\right)\right|^{4+p}\right]^{\frac{4}{4+p}} \widehat{\mathbb{Q}}^{Y_{1}, \ldots, Z_{3}}\left(\mathrm{~d}\left(\hat{y}_{1}, \ldots, \hat{z}_{3}\right)\right) \\
\leq & \int_{\mathbb{X}^{9}} \mathbb{P}\left(D_{\left(y_{1}, M_{y_{1}}\right),\left(y_{2}, M_{y_{2}}\right)}^{2} f\left(\mathcal{X}_{n-3} \cup\left\{\left(y_{3}, M_{y_{3}}\right)\right\}\right) \neq 0\right)^{\frac{p}{8+2 p}}
\end{aligned}
$$




$$
\begin{aligned}
& \times \mathbb{P}\left(D_{\left(y_{1}^{\prime}, M_{y_{1}^{\prime}}\right),\left(y_{3}^{\prime}, M_{y_{3}^{\prime}}\right)}^{2} f\left(\mathcal{X}_{n-3} \cup\left\{\left(y_{2}^{\prime}, M_{y_{2}^{\prime}}\right)\right\}\right) \neq 0\right)^{\frac{p}{8+2 p}} \\
& \times \mathbb{E}\left[\left|D_{\left(z_{2}, M_{z_{2}}\right)} f\left(\mathcal{X}_{n-3} \cup\left\{\left(z_{1}, M_{z_{1}}\right),\left(z_{3}, M_{z_{3}}\right)\right\}\right)\right|^{4+p}\right]^{\frac{4}{4+p}} \\
& \times \mathbb{Q}^{Y_{1}, \ldots, Z_{3}}\left(\mathrm{~d}\left(y_{1}, \ldots, z_{3}\right)\right) \\
\leq & c^{\frac{4}{4+p}} \int_{\mathbb{X}^{9}} \psi_{n}^{\prime}\left(y_{1}, y_{2}\right) \psi_{n}^{\prime}\left(y_{1}^{\prime}, y_{3}^{\prime}\right) \mathbb{Q}^{Y_{1}, \ldots, Z_{3}}\left(\mathrm{~d}\left(y_{1}, \ldots, z_{3}\right)\right) .
\end{aligned}
$$

If $Y_{1} \stackrel{a . s .}{=} Y_{1}^{\prime}$, this simplifies to

$$
\gamma_{Y, Y^{\prime}, Z}^{\prime}(f) \leq c^{\frac{4}{4+p}} \int_{\mathbb{X}}\left(\int_{\mathbb{X}} \psi_{n}^{\prime}\left(x, x^{\prime}\right) \mathbb{Q}\left(\mathrm{d} x^{\prime}\right)\right)^{2} \mathbb{Q}(\mathrm{d} x) .
$$

If $Y_{1}$ and $Y_{1}^{\prime}$ are independent, the Cauchy-Schwarz inequality leads to

$$
\begin{aligned}
\gamma_{Y, Y^{\prime}, Z}^{\prime}(f) & \leq c^{\frac{4}{4+p}}\left(\int_{\mathbb{X}^{2}} \psi_{n}^{\prime}\left(x, x^{\prime}\right) \mathbb{Q}^{2}\left(\mathrm{~d}\left(x, x^{\prime}\right)\right)\right)^{2} \\
& \leq c^{\frac{4}{4+p}} \int_{\mathbb{X}}\left(\int_{\mathbb{X}} \psi_{n}^{\prime}\left(x, x^{\prime}\right) \mathbb{Q}\left(\mathrm{d} x^{\prime}\right)\right)^{2} \mathbb{Q}(\mathrm{d} x) .
\end{aligned}
$$

Thus we obtain the desired bound

$$
\begin{aligned}
\frac{\sqrt{n}}{\operatorname{Var} F} \sqrt{n^{2} \gamma_{Y, Y^{\prime}, Z}^{\prime}(f)} & \leq c^{\frac{2}{4+p}} \frac{n^{\frac{3}{2}}}{\operatorname{Var} F} \sqrt{\int_{\mathbb{X}}\left(\int_{\mathbb{X}} \psi_{n}^{\prime}\left(x, x^{\prime}\right) \mathbb{Q}\left(\mathrm{d} x^{\prime}\right)\right)^{2} \mathbb{Q}(\mathrm{d} x)} \\
& \leq C(c, p) S_{2}^{\prime} .
\end{aligned}
$$

We now show that the remaining terms in (4.5) are bounded by a scalar multiple of $S_{3}^{\prime}$. For $1 \leq m \leq 4$ and $\mathbb{Q}$-a.e. $x \in \mathbb{X}$, Hölder's inequality and (4.3) lead to

$$
\begin{aligned}
& \mathbb{E}\left|D_{\left(x, M_{x}\right)} f\left(\mathcal{X}_{n-1}\right)\right|^{m} \\
& \quad \leq \mathbb{E}\left[\left|D_{\left(x, M_{x}\right)} f\left(\mathcal{X}_{n-1}\right)\right|^{4+p}\right]^{\frac{m}{4+p}} \mathbb{P}\left(D_{\left(x, M_{x}\right)} f\left(\mathcal{X}_{n-1}\right) \neq 0\right)^{\frac{4+p-m}{4+p}} \\
& \quad \leq c^{\frac{m}{4+p}} \mathbb{P}\left(D_{\left(x, M_{x}\right)} f\left(\mathcal{X}_{n-1}\right) \neq 0\right)^{\frac{p}{4+p}}
\end{aligned}
$$

where we have also used that $\frac{4+p-m}{4+p} \geq \frac{p}{4+p}$. For $1 \leq m \leq 4$ and $u \in[1 / 2,1]$, we derive from (4.6) that

$$
\begin{aligned}
& \int_{\mathbb{X}} \mathbb{E} {\left[\left|D_{\left(x, M_{x}\right)} f\left(\mathcal{X}_{n-1}\right)\right|^{m}\right]^{u} \mathbb{Q}(\mathrm{d} x) } \\
& \leq c^{\frac{m u}{4+p}} \int_{\mathbb{X}} \mathbb{P}\left(D_{\left(x, M_{x}\right)} f\left(\mathcal{X}_{n-1}\right) \neq 0\right)^{\frac{u p}{4+p}} \mathbb{Q}(\mathrm{d} x) \\
& \leq c^{\frac{m u}{4+p}} \frac{\Gamma_{n}^{\prime}}{n} .
\end{aligned}
$$


This implies immediately that, for $1 \leq m \leq 4$,

$$
\mathbb{E}\left|\mathbf{D}_{1} f(X)\right|^{m} \leq c^{\frac{m}{4+p}} \int_{\mathbb{X}} \mathbb{P}\left(D_{\left(x, M_{x}\right)} f\left(\mathcal{X}_{n-1}\right) \neq 0\right)^{\frac{p}{4+p}} \mathbb{Q}(\mathrm{d} x) \leq c^{\frac{m}{4+p}} \frac{\Gamma_{n}^{\prime}}{n} .
$$

This gives for $m=4$ and $m=3$ that the third and fifth terms in (4.5) are bounded by

$$
\frac{\sqrt{n} \sqrt{\mathbb{E} \mathbf{D}_{1} f(X)^{4}}}{\operatorname{Var} F}+\frac{n \mathbb{E}\left|\mathbf{D}_{1} f(X)\right|^{3}}{(\operatorname{Var} F)^{\frac{3}{2}}} \leq \frac{c^{\frac{2}{4+p}} \sqrt{\Gamma_{n}^{\prime}}}{\operatorname{Var} F}+\frac{c^{\frac{3}{4+p}} \Gamma_{n}^{\prime}}{(\operatorname{Var} F)^{\frac{3}{2}}} \leq C(c, p) S_{3}^{\prime} .
$$

Lastly, we bound the fourth term in (4.5) by a scalar multiple of $S_{3}^{\prime}$. Let $Y$ be a recombination of $\left\{X, X^{\prime}, \tilde{X}\right\}$. Noting that $Y \stackrel{(d)}{=} X$, let us estimate

$$
\begin{aligned}
& \mathbb{E}\left|(f(X)-\mathbb{E} F)\left(\mathbf{D}_{1} f(Y)\right)^{3}\right| \\
&=\mathbb{E}\left|\left(f\left(X^{1}\right)-\mathbb{E} F+\mathbf{D}_{1} f(X)\right)\left(\mathbf{D}_{1} f(Y)\right)^{3}\right| \\
& \leq \int_{\mathbb{X}} \mathbb{E}\left[\left|f\left(X^{1}\right)-\mathbb{E} F\right|\left|D_{\left(y_{1}, M_{y_{1}}\right)} f\left(Y^{1}\right)\right|^{3}\right] \mathbb{Q}\left(\mathrm{d} y_{1}\right) \\
&+\mathbb{E}\left[\left|\mathbf{D}_{1} f(X)\right|\left|\mathbf{D}_{1} f(Y)\right|^{3}\right] \\
& \leq \mathbb{E}\left[\left(f\left(\mathcal{X}_{n}^{1}\right)-\mathbb{E} F\right)^{4}\right]^{\frac{1}{4}} \int_{\mathbb{X}} \mathbb{E}\left[\left(D_{\left(x, M_{x}\right)} f\left(\mathcal{X}_{n-1}\right)\right)^{4}\right]^{\frac{3}{4}} \mathbb{Q}(\mathrm{d} x) \\
&+\mathbb{E}\left(\mathbf{D}_{1} f(X)\right)^{4} \\
& \leq\left(\mathbb{E}\left[\left(f\left(\mathcal{X}_{n}\right)-\mathbb{E} F\right)^{4}\right]^{\frac{1}{4}}\right. \\
&\left.+\mathbb{E}\left[\left(\mathbf{D}_{1} f(X)\right)^{4}\right]^{\frac{1}{4}}\right) \int_{\mathbb{X}} \mathbb{E}\left[\left(D_{\left(x, M_{x}\right)} f\left(\mathcal{X}_{n-1}\right)\right)^{4}\right]^{\frac{3}{4}} \mathbb{Q}(\mathrm{d} x)+c^{\frac{4}{4+p}} \frac{\Gamma_{n}^{\prime}}{n} .
\end{aligned}
$$

By (4.7), we have

$$
\int_{\mathbb{X}} \mathbb{E}\left[\left(D_{\left(x, M_{x}\right)} f\left(\mathcal{X}_{n-1}\right)\right)^{4}\right]^{\frac{3}{4}} \mathbb{Q}(\mathrm{d} x) \leq c^{\frac{3}{4+p}} \frac{\Gamma_{n}^{\prime}}{n} .
$$

From Lemma 4.4 and (4.7), it follows that

$$
\begin{aligned}
\frac{\mathbb{E}(F-\mathbb{E} F)^{4}}{(\operatorname{Var} F)^{2}} \leq & 9 \max \left\{\left(\frac{32 n}{\operatorname{Var} F} \int_{\mathbb{X}} \sqrt{\mathbb{E}\left(D_{\left(y, M_{y}\right)} f\left(\mathcal{X}_{n-1}\right)\right)^{4}} \mathbb{Q}(\mathrm{d} y)\right)^{2},\right. \\
& \left.4 n \frac{\mathbb{E}\left(\mathbf{D}_{1} f\left(\mathcal{X}_{n}\right)\right)^{4}}{(\operatorname{Var} F)^{2}}+1\right\} \\
\leq & 9 \max \left\{\frac{1024 c^{\frac{4}{4+p}}\left(\Gamma_{n}^{\prime}\right)^{2}}{(\operatorname{Var} F)^{2}}, \frac{4 c^{\frac{4}{4+p}} \Gamma_{n}^{\prime}}{(\operatorname{Var} F)^{2}}+1\right\} .
\end{aligned}
$$


Altogether, the fourth term in (4.5) satisfies the bound

$$
\begin{aligned}
& \frac{n \mathbb{E}\left|(f(X)-\mathbb{E} F)\left(\mathbf{D}_{1} f(Y)\right)^{3}\right|}{(\operatorname{Var} F)^{2}} \\
& \leq\left(\sqrt { 3 } \operatorname { m a x } \left\{\frac{4 \cdot \sqrt{2} c^{\frac{1}{4+p}} \sqrt{\Gamma_{n}^{\prime}}}{\sqrt{\operatorname{Var} F}}, \frac{\sqrt{2} c^{\frac{1}{4+p}} \Gamma_{n}^{\prime \frac{1}{4}}}{\sqrt{\operatorname{Var} F}+1\}}\right.\right. \\
& \left.\quad+\frac{c^{\frac{1}{4+p}} \Gamma_{n}^{\prime \frac{1}{4}}}{n^{\frac{1}{4}} \sqrt{\operatorname{Var} F}}\right) \frac{c^{\frac{3}{4+p}} \Gamma_{n}^{\prime}}{(\operatorname{Var} F)^{\frac{3}{2}}}+\frac{c^{\frac{4}{4+p}} \Gamma_{n}^{\prime}}{(\operatorname{Var} F)^{2}} \\
& \leq C(c, p) S_{3}^{\prime},
\end{aligned}
$$

which completes the proof.

REMARK. The bounds in Theorem 4.1 and Theorem 4.2 are still valid for the Wasserstein distance given in (2.21). This follows from the fact that the underlying bounds in Theorem 6.1 in [22] and Theorem 4.3 (see also Remark 4.3 in [20]) are true for the Wasserstein distance as well.

5. Proofs of Theorem 2.1 and Theorem 2.3. The bounds in Theorems 4.1 and 4.2 are admittedly unwieldy. However, when $F$ is a sum of stabilizing score functions, as in (1.1) and (1.2), then the terms on the right-hand side of (4.2) and (4.4) conveniently collapse into the more manageable bounds (2.11) and (2.12), respectively.

We first provide several lemmas giving moment and probability bounds for the first- and second-order difference operators. Throughout we assume that the hypotheses of Theorem 2.1 are in force. We can assume without loss of generality that $C_{\text {stab }}=C_{K}=: C, c_{\text {stab }}=c_{K}=: c$ and $\alpha_{\text {stab }}=\alpha_{K}=: \alpha$.

LEMMA 5.1. (a) For any $x \in \mathbb{X}$ and $r \geq 0$,

$$
\mathbb{Q}(B(x, r)) \leq \kappa r^{\gamma} .
$$

(b) For any $v>0$, there is a constant $C_{v} \in(0, \infty)$ such that

$$
\int_{\mathbb{X} \backslash B(x, r)} \exp \left(-\left(\beta^{1 / \gamma} \mathrm{d}(x, y)\right)^{v}\right) \mathbb{Q}(\mathrm{d} y) \leq \frac{C_{v}}{\beta} \exp \left(-\left(\beta^{1 / \gamma} r\right)^{v} / 2\right)
$$

for all $\beta \geq 1, x \in \mathbb{X}$ and $r \geq 0$.

ProOF. We prove only (b) since (a) can be shown similarly. We first derive the inequality

$$
\mathbb{Q}(B(x, v))-\mathbb{Q}(B(x, u)) \leq \kappa \gamma \max \left\{u^{\gamma-1}, v^{\gamma-1}\right\}(v-u)
$$


for $0<u<v<\infty$. Let $g(t):=\mathbb{Q}(B(x, t)), t>0$, and assume that there is a $c \in(0, \infty)$ such that $g(v)-g(u) \geq c(v-u)$. Then one can construct sequences $\left(u_{n}\right)_{n \in \mathbb{N}}$ and $\left(v_{n}\right)_{n \in \mathbb{N}}$ such that $u_{1}=u, v_{1}=v, u_{n} \leq u_{n+1}<v_{n+1} \leq v_{n}, n \in \mathbb{N}$, $\lim _{n \rightarrow \infty} u_{n}=\lim _{n \rightarrow \infty} v_{n}=: w$, and $g\left(v_{n}\right)-g\left(u_{n}\right) \geq c\left(v_{n}-u_{n}\right), n \in \mathbb{N}$. Consequently,

$$
\begin{aligned}
c & \leq \frac{v_{n}-w}{v_{n}-u_{n}} \frac{g\left(v_{n}\right)-g(w)}{v_{n}-w}+\frac{w-u_{n}}{v_{n}-u_{n}} \frac{g(w)-g\left(u_{n}\right)}{w-u_{n}} \\
& \leq \max \left\{\frac{g\left(v_{n}\right)-g(w)}{v_{n}-w}, \frac{g(w)-g\left(u_{n}\right)}{w-u_{n}}\right\}
\end{aligned}
$$

and $n \rightarrow \infty$ and (2.1) lead to $c \leq \kappa \gamma w^{\gamma-1} \leq \kappa \gamma \max \left\{u^{\gamma-1}, v^{\gamma-1}\right\}$.

It is sufficient to show (5.2) for $r>0$ since the case $r=0$ then follows from $r \rightarrow 0$. For any monotone sequence $\left(r_{n}\right)_{n \in \mathbb{N}}$ with $r_{1}>r=: r_{0}$ and $\lim _{n \rightarrow \infty} r_{n}=$ $\infty$, we have

$$
\begin{aligned}
J_{r} & :=\int_{\mathbb{X} \backslash B(x, r)} \exp \left(-\left(\beta^{1 / \gamma} \mathrm{d}(x, y)\right)^{\nu}\right) \mathbb{Q}(\mathrm{d} y) \\
& \leq \sum_{n=1}^{\infty} \exp \left(-\left(\beta^{1 / \gamma_{r_{n-1}}}\right)^{\nu}\right) \mathbb{Q}\left(B\left(x, r_{n}\right) \backslash B\left(x, r_{n-1}\right)\right) .
\end{aligned}
$$

For $\sup _{n \in \mathbb{N}}\left|r_{n}-r_{n-1}\right| \rightarrow 0$, the inequality (5.3) and the properties of the Riemann integral imply that

$$
J_{r} \leq \int_{r}^{\infty} \exp \left(-\left(\beta^{1 / \gamma} u\right)^{\nu}\right) \kappa \gamma u^{\gamma-1} \mathrm{~d} u=\frac{1}{\beta} \int_{\beta^{1 / \gamma_{r}}}^{\infty} \exp \left(-w^{\nu}\right) \kappa \gamma w^{\gamma-1} \mathrm{~d} w .
$$

Now a straightforward computation completes the proof of (b).

Throughout our proofs, we only make use of (5.1) and (5.2) and not of (2.1) so that one could replace the assumption (2.1) by (5.1) and (5.2).

LEMmA 5.2. Let $\mathcal{M} \in \mathbf{N}$ and $\hat{y}, \hat{y}_{1}, \hat{y}_{2} \in \widehat{\mathbb{X}}$. Then, for $s \geq 1$,

$$
\begin{aligned}
D_{\hat{y}} h_{s}(\mathcal{M})= & \xi_{s}(\hat{y}, \mathcal{M} \cup\{\hat{y}\})+\sum_{x \in \mathcal{M}} D_{\hat{y}} \xi_{s}(x, \mathcal{M}), \\
D_{\hat{y}_{1}, \hat{y}_{2}}^{2} h_{s}(\mathcal{M})= & D_{\hat{y}_{1}} \xi_{s}\left(\hat{y}_{2}, \mathcal{M} \cup\left\{\hat{y}_{2}\right\}\right)+D_{\hat{y}_{2}} \xi_{s}\left(\hat{y}_{1}, \mathcal{M} \cup\left\{\hat{y}_{1}\right\}\right) \\
& +\sum_{x \in \mathcal{M}} D_{\hat{y}_{1}, \hat{y}_{2}}^{2} \xi_{s}(x, \mathcal{M}) .
\end{aligned}
$$

Proof. In the following, let $h:=h_{s}$ and $\xi:=\xi_{s}$. By the definition of the difference operator, we have that

$$
D_{\hat{y}} h(\mathcal{M})=\sum_{x \in \mathcal{M} \cup\{\hat{y}\}} \xi(x, \mathcal{M} \cup\{\hat{y}\})-\sum_{x \in \mathcal{M}} \xi(x, \mathcal{M})
$$




$$
\begin{aligned}
& =\xi(\hat{y}, \mathcal{M} \cup\{\hat{y}\})+\sum_{x \in \mathcal{M}}(\xi(x, \mathcal{M} \cup\{\hat{y}\})-\xi(x, \mathcal{M})) \\
& =\xi(\hat{y}, \mathcal{M} \cup\{\hat{y}\})+\sum_{x \in \mathcal{M}} D_{\hat{y}} \xi(x, \mathcal{M}) .
\end{aligned}
$$

For the second-order difference operator, this implies that

$$
\begin{array}{rl}
D_{\hat{y}_{1}, \hat{y}_{2}}^{2} & h(\mathcal{M}) \\
= & \xi\left(\hat{y}_{2}, \mathcal{M} \cup\left\{\hat{y}_{1}, \hat{y}_{2}\right\}\right)+\sum_{x \in \mathcal{M} \cup\left\{\hat{y}_{1}\right\}} D_{\hat{y}_{2}} \xi\left(x, \mathcal{M} \cup\left\{\hat{y}_{1}\right\}\right) \\
& -\xi\left(\hat{y}_{2}, \mathcal{M} \cup\left\{\hat{y}_{2}\right\}\right)-\sum_{x \in \mathcal{M}} D_{\hat{y}_{2}} \xi(x, \mathcal{M}) \\
= & D_{\hat{y}_{1}} \xi\left(\hat{y}_{2}, \mathcal{M} \cup\left\{\hat{y}_{2}\right\}\right)+D_{\hat{y}_{2}} \xi\left(\hat{y}_{1}, \mathcal{M} \cup\left\{\hat{y}_{1}\right\}\right) \\
& +\sum_{x \in \mathcal{M}}\left(D_{\hat{y}_{2}} \xi\left(x, \mathcal{M} \cup\left\{\hat{y}_{1}\right\}\right)-D_{\hat{y}_{2}} \xi(x, \mathcal{M})\right) \\
= & D_{\hat{y}_{1}} \xi\left(\hat{y}_{2}, \mathcal{M} \cup\left\{\hat{y}_{2}\right\}\right)+D_{\hat{y}_{2}} \xi\left(\hat{y}_{1}, \mathcal{M} \cup\left\{\hat{y}_{1}\right\}\right)+\sum_{x \in \mathcal{M}} D_{\hat{y}_{1}, \hat{y}_{2}}^{2} \xi(x, \mathcal{M}),
\end{array}
$$

which completes the proof.

If a point $\hat{y} \in \widehat{\mathbb{X}}$ is inserted into $\mathcal{M} \in \mathbf{N}$ at a distance exceeding the stabilization radius at $\hat{x} \in \mathcal{M}$, then the difference operator $D_{\hat{y}}$ of the score at $\hat{x}$ vanishes, as seen by the next lemma.

Lemma 5.3. Let $\mathcal{M} \in \mathbf{N},\left(x, m_{x}\right) \in \mathcal{M}, \widehat{\mathcal{A}} \subset \widehat{\mathbb{X}}$ with $|\widehat{\mathcal{A}}| \leq 6, y, y_{1}, y_{2} \in \mathbb{X}$ and $m_{y}, m_{y_{1}}, m_{y_{2}} \in \mathbb{M}$. Then, for $s \geq 1$,

$$
D_{\left(y, m_{y}\right)} \xi_{s}\left(\left(x, m_{x}\right), \mathcal{M} \cup \widehat{\mathcal{A}}\right)=0
$$

if $R_{S}\left(\left(x, m_{x}\right), \mathcal{M}\right)<\mathrm{d}(x, y)$ and

$$
D_{\left(y_{1}, m_{y_{1}}\right),\left(y_{2}, m_{y_{2}}\right)}^{2} \xi_{s}\left(\left(x, m_{x}\right), \mathcal{M}\right)=0
$$

if $R_{S}\left(\left(x, m_{x}\right), \mathcal{M}\right)<\max \left\{\mathrm{d}\left(x, y_{1}\right), \mathrm{d}\left(x, y_{2}\right)\right\}$.

Proof. Note that $R:=R_{s}$ and $\xi:=\xi_{s}$. Moreover, we use the abbreviations $\hat{x}:=\left(x, m_{x}\right), \hat{y}:=\left(y, m_{y}\right), \hat{y}_{1}:=\left(y_{1}, m_{y_{1}}\right)$ and $\hat{y}_{2}:=\left(y_{2}, m_{y_{2}}\right)$. Recall that $\widehat{B}(z, r)$ stands for the cylinder $B(z, r) \times \mathbb{M}$ for $z \in \mathbb{X}$ and $r>0$. It follows from the definitions of the difference operator and of the radius of stabilization that

$$
\begin{aligned}
D_{\hat{y}} \xi(\hat{x}, \mathcal{M} \cup \widehat{\mathcal{A}})= & \xi(\hat{x}, \mathcal{M} \cup \widehat{\mathcal{A}} \cup\{\hat{y}\})-\xi(\hat{x}, \mathcal{M} \cup \widehat{\mathcal{A}}) \\
= & \xi(\hat{x},(\mathcal{M} \cup \widehat{\mathcal{A}} \cup\{\hat{y}\}) \cap \widehat{B}(x, R(\hat{x}, \mathcal{M}))) \\
& -\xi(\hat{x},(\mathcal{M} \cup \widehat{\mathcal{A}}) \cap \widehat{B}(x, R(\hat{x}, \mathcal{M}))) .
\end{aligned}
$$


If $R(\hat{x}, \mathcal{M})<\mathrm{d}(x, y)$, we have

$$
(\mathcal{M} \cup \widehat{\mathcal{A}} \cup\{\hat{y}\}) \cap \widehat{B}(x, R(\hat{x}, \mathcal{M}))=(\mathcal{M} \cup \widehat{\mathcal{A}}) \cap \widehat{B}(x, R(\hat{x}, \mathcal{M}))
$$

so that the terms on the right-hand side of (5.4) cancel out. For the second-order difference operator, we obtain that

$$
\begin{aligned}
D_{\hat{y}_{1}, \hat{y}_{2}}^{2} \xi(\hat{x}, \mathcal{M}) & \\
(5.5)= & \xi\left(\hat{x},\left(\mathcal{M} \cup\left\{\hat{y}_{1}, \hat{y}_{2}\right\}\right) \cap \widehat{B}\left(x, R\left(-\xi\left(\hat{x},\left(\mathcal{M} \cup\left\{\hat{y}_{1}\right\}\right) \cap \widehat{B}(x, R(\hat{x}, \mathcal{M}))\right)\right.\right.\right. \\
& -\xi\left(\hat{x},\left(\mathcal{M} \cup\left\{\hat{y}_{2}\right\}\right) \cap \widehat{B}(x, R(\hat{x}, \mathcal{M}))\right)+\xi(\hat{x}, \mathcal{M} \cap \widehat{B}(x, R(\hat{x}, \mathcal{M}))) .
\end{aligned}
$$

Without loss of generality, we can assume that $\mathrm{d}\left(x, y_{1}\right) \geq \mathrm{d}\left(x, y_{2}\right)$. If $R(\hat{x}, \mathcal{M})<$ $\max \left\{\mathrm{d}\left(x, y_{1}\right), \mathrm{d}\left(x, y_{2}\right)\right\}=\mathrm{d}\left(x, y_{1}\right)$, we see that

$$
\left(\mathcal{M} \cup\left\{y_{1}, y_{2}\right\}\right) \cap \widehat{B}(x, R(\hat{x}, \mathcal{M}))=\left(\mathcal{M} \cup\left\{y_{2}\right\}\right) \cap \widehat{B}(x, R(\hat{x}, \mathcal{M}))
$$

and

$$
\left(\mathcal{M} \cup\left\{\hat{y}_{1}\right\}\right) \cap \widehat{B}(x, R(\hat{x}, \mathcal{M}))=\mathcal{M} \cap \widehat{B}(x, R(\hat{x}, \mathcal{M})),
$$

whence the terms on the right-hand side of (5.5) cancel out.

We recall that $M_{x}, x \in \mathbb{X}$, always stands for a random mark distributed according to $\mathbb{Q}_{\mathbb{M}}$ and associated with the point $x$. Moreover, we tacitly assume that $M_{x}$ is independent from everything else. For a finite set $\mathcal{A} \subset \mathbb{X},\left(\mathcal{A}, M_{\mathcal{A}}\right)$ is the shorthand notation for $\left\{\left(x, M_{x}\right): x \in \mathcal{A}\right\}$. The next lemma shows that moments of difference operators of the scores are uniformly bounded. In the following, $p \in(0,1]$ and $C_{p}>0$ come from the moment assumptions (2.6) and (2.7), respectively.

Lemma 5.4. (a) For any $\varepsilon \in(0, p]$ and for all $s \geq 1, x, y \in \mathbb{X}$ and $\mathcal{A} \subset \mathbb{X}$ with $|\mathcal{A}| \leq 6$,

$$
\mathbb{E}\left|D_{\left(y, M_{y}\right)} \xi_{s}\left(\left(x, M_{x}\right), \mathcal{P}_{s} \cup\left\{\left(x, M_{x}\right)\right\} \cup\left(\mathcal{A}, M_{\mathcal{A}}\right)\right)\right|^{4+\varepsilon} \leq 2^{4+\varepsilon} C_{p}^{\frac{4+\varepsilon}{4+p}} .
$$

(b) For any $\varepsilon \in(0, p]$ and for all $n \geq 9, x, y \in \mathbb{X}$ and $\mathcal{A} \subset \mathbb{X}$ with $|\mathcal{A}| \leq 6$,

$$
\mathbb{E}\left|D_{\left(y, M_{y}\right)} \xi_{n}\left(\left(x, M_{x}\right), \mathcal{X}_{n-8} \cup\left\{\left(x, M_{x}\right)\right\} \cup\left(\mathcal{A}, M_{\mathcal{A}}\right)\right)\right|^{4+\varepsilon} \leq 2^{4+\varepsilon} C_{p}^{\frac{4+\varepsilon}{4+p}} .
$$

PROOF. It follows from Jensen's inequality, Hölder's inequality and (2.6) that

$$
\begin{aligned}
\mathbb{E}\left|D_{\left(y, M_{y}\right)} \xi_{s}\left(\left(x, M_{x}\right), \mathcal{P}_{s} \cup\left(\mathcal{A}, M_{\mathcal{A}}\right) \cup\left\{\left(x, M_{x}\right)\right\}\right)\right|^{4+\varepsilon} \\
\leq 2^{3+\varepsilon} \mathbb{E}\left(\left|\xi_{s}\left(\left(x, M_{x}\right), \mathcal{P}_{s} \cup\left(\mathcal{A}, M_{\mathcal{A}}\right) \cup\left\{\left(y, M_{y}\right)\right\} \cup\left\{\left(x, M_{x}\right)\right\}\right)\right|^{4+\varepsilon}\right. \\
\left.\quad+\left|\xi_{s}\left(\left(x, M_{x}\right), \mathcal{P}_{s} \cup\left(\mathcal{A}, M_{\mathcal{A}}\right) \cup\left\{\left(x, M_{x}\right)\right\}\right)\right|^{4+\varepsilon}\right) \\
\leq 2^{4+\varepsilon} C_{p}^{\frac{4+\varepsilon}{4+p}}
\end{aligned}
$$

which proves (a). Part (b) follows in the same way from (2.7). 
LEMMA 5.5. For any $\varepsilon \in(0, p)$, there is a constant $C_{\varepsilon} \in(0, \infty)$ only depending on the constants in (2.1), (2.4) and (2.6) such that

$$
\mathbb{E}\left|D_{\left(y, M_{y}\right)} h_{s}\left(\mathcal{P}_{s} \cup\left(\mathcal{A}, M_{\mathcal{A}}\right)\right)\right|^{4+\varepsilon} \leq C_{\varepsilon}
$$

for $y \in \mathbb{X}, \mathcal{A} \subset \mathbb{X}$ with $|\mathcal{A}| \leq 1$ and $s \geq 1$.

Proof. Fix $y \in \mathbb{X}$. We start with the case $\mathcal{A}=\varnothing$. It follows from Lemma 5.2 and Jensen's inequality that

$$
\begin{aligned}
\mathbb{E}\left|D_{\left(y, M_{y}\right)} h_{s}\left(\mathcal{P}_{s}\right)\right|^{4+\varepsilon} & \\
= & \mathbb{E}\left|\xi_{s}\left(\left(y, M_{y}\right), \mathcal{P}_{s} \cup\left\{\left(y, M_{y}\right)\right\}\right)+\sum_{x \in \mathcal{P}_{s}} D_{\left(y, M_{y}\right)} \xi_{s}\left(x, \mathcal{P}_{s}\right)\right|^{4+\varepsilon} \\
\leq & 2^{3+\varepsilon} \mathbb{E}\left|\xi_{s}\left(\left(y, M_{y}\right), \mathcal{P}_{s} \cup\left\{\left(y, M_{y}\right)\right\}\right)\right|^{4+\varepsilon} \\
& +2^{3+\varepsilon} \mathbb{E}\left|\sum_{x \in \mathcal{P}_{s}} D_{\left(y, M_{y}\right)} \xi_{s}\left(x, \mathcal{P}_{s}\right)\right|^{4+\varepsilon} .
\end{aligned}
$$

Here, the first summand is bounded by $2^{3+\varepsilon}\left(C_{p}+1\right)$ by assumption (2.6). The second summand is a sum of $Z:=\sum_{x \in \mathcal{P}_{s}} \mathbf{1}_{\left\{D_{\left(y, M_{y}\right)} \xi_{s}\left(x, \mathcal{P}_{s}\right) \neq 0\right\}}$ terms distinct from zero. A further application of Jensen's inequality to the function $x \mapsto x^{4+\varepsilon}$ leads to

$$
\begin{aligned}
\left|\sum_{x \in \mathcal{P}_{s}} D_{\left(y, M_{y}\right)} \xi_{s}\left(x, \mathcal{P}_{s}\right)\right|^{4+\varepsilon} & \leq Z^{4+\varepsilon}\left|\sum_{x \in \mathcal{P}_{s}} Z^{-1} D_{\left(y, M_{y}\right)} \xi_{s}\left(x, \mathcal{P}_{s}\right)\right|^{4+\varepsilon} \\
& \leq Z^{4+\varepsilon} \sum_{x \in \mathcal{P}_{s}} Z^{-1}\left|D_{\left(y, M_{y}\right)} \xi_{s}\left(x, \mathcal{P}_{s}\right)\right|^{4+\varepsilon} \\
& \leq Z^{4} \sum_{x \in \mathcal{P}_{s}}\left|D_{\left(y, M_{y}\right)} \xi_{s}\left(x, \mathcal{P}_{s}\right)\right|^{4+\varepsilon} .
\end{aligned}
$$

By deciding whether points in different sums are identical or distinct, we obtain that

$$
\mathbb{E} Z^{4} \sum_{x \in \mathcal{P}_{s}}\left|D_{\left(y, M_{y}\right)} \xi_{s}\left(x, \mathcal{P}_{s}\right)\right|^{4+\varepsilon}=I_{1}+15 I_{2}+25 I_{3}+10 I_{4}+I_{5},
$$

where, for $i \in\{1, \ldots, 5\}$,

$$
I_{i}=\mathbb{E} \sum_{\left(x_{1}, \ldots, x_{i}\right) \in \mathcal{P}_{s, \neq}^{i}} \mathbf{1}_{\left\{D_{\left(y, M_{y}\right)} \xi_{s}\left(x_{j}, \mathcal{P}_{s}\right) \neq 0, j=1, \ldots, i\right\}}\left|D_{\left(y, M_{y}\right)} \xi_{s}\left(x_{1}, \mathcal{P}_{s}\right)\right|^{4+\varepsilon}
$$


Here, we denote by $\mathcal{P}_{s, \neq}^{i}$ the set of $i$-tuples of distinct points of $\mathcal{P}_{s}$. It follows from the multivariate Mecke formula and Hölder's inequality that

$$
\begin{aligned}
I_{i}= & s^{i} \int_{\widehat{\mathbb{X}}^{i}} \mathbb{E}\left[\mathbf{1}_{\left\{D_{\left(y, M_{y}\right)} \xi_{s}\left(x_{j}, \mathcal{P}_{s} \cup\left\{x_{1}, \ldots, x_{i}\right\}\right) \neq 0, j=1, \ldots, i\right\}}\right. \\
& \left.\times\left|D_{\left(y, M_{y}\right)} \xi_{s}\left(x_{1}, \mathcal{P}_{s} \cup\left\{x_{1}, \ldots, x_{i}\right\}\right)\right|^{4+\varepsilon}\right] \widehat{\mathbb{Q}}^{i}\left(\mathrm{~d}\left(x_{1}, \ldots, x_{i}\right)\right) \\
\leq & s^{i} \int_{\mathbb{X}^{i}} \prod_{j=1}^{i} \mathbb{P}\left(D_{\left(y, M_{y}\right)} \xi_{s}\left(x_{j}, \mathcal{P}_{s} \cup\left\{\left(x_{1}, M_{x_{1}}\right), \ldots,\left(x_{i}, M_{x_{i}}\right)\right\}\right) \neq 0\right)^{\frac{p-\varepsilon}{4 i+p i}} \\
& \times\left(\mathbb{E}\left|D_{\left(y, M_{y}\right)} \xi_{s}\left(x_{1}, \mathcal{P}_{s} \cup\left\{\left(x_{1}, M_{x_{1}}\right), \ldots,\left(x_{i}, M_{x_{i}}\right)\right\}\right)\right|^{4+p}\right)^{\frac{4+\varepsilon}{4+p}} \\
& \times \mathbb{Q}^{i}\left(\mathrm{~d}\left(x_{1}, \ldots, x_{i}\right)\right) .
\end{aligned}
$$

Combining this with Lemma 5.3, (2.4) and Lemma 5.4(a) leads to

$$
\begin{aligned}
I_{i} & \leq 2^{4+\varepsilon} C_{p}^{\frac{4+\varepsilon}{4+p}} s^{i} \int_{\mathbb{X}^{i}} C^{\frac{p-\varepsilon}{4+p}} \prod_{j=1}^{i} \exp \left(-\frac{c(p-\varepsilon)}{4 i+p i} \mathrm{~d}_{s}\left(x_{j}, y\right)^{\alpha}\right) \mathbb{Q}^{i}\left(\mathrm{~d}\left(x_{1}, \ldots, x_{i}\right)\right) \\
& =2^{4+\varepsilon} C_{p}^{\frac{4+\varepsilon}{4+p}}\left(s C^{\frac{p-\varepsilon}{4 i+p i}} \int_{\mathbb{X}} \exp \left(-\frac{c(p-\varepsilon)}{4 i+p i} \mathrm{~d}_{s}(x, y)^{\alpha}\right) \mathbb{Q}(\mathrm{d} x)\right)^{i} .
\end{aligned}
$$

Now (5.2) with $r=0$ yields that the integrals on the right-hand side are uniformly bounded, and thus the first asserted moment bound holds.

Next, we assume that $\mathcal{A}=\{z\}$ with $z \in \mathbb{X}$. Lemma 5.2 and a further application of Jensen's inequality show that

$$
\begin{aligned}
& \mathbb{E}\left|D_{\left(y, M_{y}\right)} h_{s}\left(\mathcal{P}_{s} \cup\left\{\left(z, M_{z}\right)\right\}\right)\right|^{4+\varepsilon} \\
&=\mathbb{E} \mid \xi_{s}\left(\left(y, M_{y}\right), \mathcal{P}_{s} \cup\left\{\left(y, M_{y}\right),\left(z, M_{z}\right)\right\}\right) \\
&+D_{\left(y, M_{y}\right)} \xi_{s}\left(\left(z, M_{z}\right), \mathcal{P}_{s} \cup\left\{\left(z, M_{z}\right)\right\}\right) \\
&+\left.\sum_{x \in \mathcal{P}_{s}} D_{\left(y, M_{y}\right)} \xi_{s}\left(x, \mathcal{P}_{s} \cup\left\{\left(z, M_{z}\right)\right\}\right)\right|^{4+\varepsilon} \\
& \leq 3^{3+\varepsilon} \mathbb{E}\left|\xi_{s}\left(\left(y, M_{y}\right), \mathcal{P}_{s} \cup\left\{\left(y, M_{y}\right),\left(z, M_{z}\right)\right\}\right)\right|^{4+\varepsilon} \\
&+3^{3+\varepsilon} \mathbb{E}\left|D_{\left(y, M_{y}\right)} \xi_{s}\left(\left(z, M_{z}\right), \mathcal{P}_{s} \cup\left\{\left(z, M_{z}\right)\right\}\right)\right|^{4+\varepsilon} \\
&+3^{3+\varepsilon} \mathbb{E}\left|\sum_{x \in \mathcal{P}_{s}} D_{\left(y, M_{y}\right)} \xi_{s}\left(x, \mathcal{P}_{s} \cup\left\{\left(z, M_{z}\right)\right\}\right)\right|^{4+\varepsilon} .
\end{aligned}
$$

The last term on the right-hand side can be now bounded by exactly the same arguments as above since these still hold true if one adds an additional point. As the other terms are bounded by (2.6) and Lemma 5.4(a), this completes the proof. 
Lemma 5.6. For any $\varepsilon \in(0, p)$, there is a constant $C_{\varepsilon} \in(0, \infty)$ only depending on the constants in (2.1), (2.5) and (2.7) such that

$$
\mathbb{E}\left|D_{\left(y, M_{y}\right)} h_{n}\left(\mathcal{X}_{n-1-|\mathcal{A}|} \cup\left(\mathcal{A}, M_{\mathcal{A}}\right)\right)\right|^{4+\varepsilon} \leq C_{\varepsilon}
$$

for $y \in \mathbb{X}, \mathcal{A} \subset \mathbb{X}$ with $|\mathcal{A}| \leq 2$ and $n \geq 9$.

PRoOf. Let $\mathcal{X}_{n, \mathcal{A}}:=\mathcal{X}_{n-1-|\mathcal{A}|} \cup\left(\mathcal{A}, M_{\mathcal{A}}\right)$. It follows from Lemma 5.2 and Jensen's inequality that

$$
\begin{aligned}
& \mathbb{E}\left|D_{\left(y, M_{y}\right)} h_{n}\left(\mathcal{X}_{n, \mathcal{A}}\right)\right|^{4+\varepsilon} \\
&= \mathbb{E} \mid \xi_{n}\left(\left(y, M_{y}\right), \mathcal{X}_{n, \mathcal{A}} \cup\left\{\left(y, M_{y}\right)\right\}\right) \\
&+\left.\sum_{x \in \mathcal{X}_{n-1-|\mathcal{A}|} \cup\left(\mathcal{A}, M_{\mathcal{A}}\right)} D_{\left(y, M_{y}\right)} \xi_{n}\left(x, \mathcal{X}_{n, \mathcal{A}}\right)\right|^{4+\varepsilon} \\
& \leq 4^{3+\varepsilon} \mathbb{E}\left|\xi_{n}\left(\left(y, M_{y}\right), \mathcal{X}_{n, \mathcal{A}} \cup\left\{\left(y, M_{y}\right)\right\}\right)\right|^{4+\varepsilon} \\
&+4^{3+\varepsilon} \sum_{x \in \mathcal{A}} \mathbb{E}\left|D_{\left(y, M_{y}\right)} \xi_{n}\left(\left(x, M_{x}\right), \mathcal{X}_{n, \mathcal{A}}\right)\right|^{4+\varepsilon} \\
&+\left.\left.4^{3+\varepsilon} \mathbb{E}\right|_{x \in \mathcal{X}_{n-1-|\mathcal{A}|}} D_{\left(y, M_{y}\right)} \xi_{n}\left(x, \mathcal{X}_{n, \mathcal{A}}\right)\right|^{4+\varepsilon} .
\end{aligned}
$$

On the right-hand side, the first summand is bounded by $4^{3+\varepsilon}\left(C_{p}+1\right)$ by assumption (2.7) (after conditioning on the points of $\mathcal{X}_{n-1-|\mathcal{A}|} \backslash \mathcal{X}_{n-8}$ ) and the second summand is bounded by $4^{3+\varepsilon} \cdot 2 \cdot 2^{4+\varepsilon} C_{p}^{\frac{4+\varepsilon}{4+p}}$ by Lemma 5.4(b). A further application of Jensen's inequality with $Z:=\sum_{x \in \mathcal{X}_{n-1-|\mathcal{A}|}} \mathbf{1}_{\left.\left\{D_{\left(y, M_{y}\right.}\right) \xi_{n}\left(x, \mathcal{X}_{n, \mathcal{A}}\right) \neq 0\right\}}$ leads to

$$
\begin{aligned}
\left|\sum_{x \in \mathcal{X}_{n-1-|\mathcal{A}|}} D_{\left(y, M_{y}\right)} \xi_{n}\left(x, \mathcal{X}_{n, \mathcal{A}}\right)\right|^{4+\varepsilon} & \leq Z^{3+\varepsilon} \sum_{x \in \mathcal{X}_{n-1-|\mathcal{A}|}}\left|D_{\left(y, M_{y}\right)} \xi_{n}\left(x, \mathcal{X}_{n, \mathcal{A}}\right)\right|^{4+\varepsilon} \\
& \leq Z^{4} \sum_{x \in \mathcal{X}_{n-1-|\mathcal{A}|}}\left|D_{\left(y, M_{y}\right)} \xi_{n}\left(x, \mathcal{X}_{n, \mathcal{A}}\right)\right|^{4+\varepsilon} .
\end{aligned}
$$

By deciding whether points in different sums are identical or distinct, we obtain that

$$
\mathbb{E} Z^{4} \sum_{x \in \mathcal{X}_{n-1-|\mathcal{A}|}}\left|D_{\left(y, M_{y}\right)} \xi_{n}\left(x, \mathcal{X}_{n, \mathcal{A}}\right)\right|^{4+\varepsilon}=I_{1}+15 I_{2}+25 I_{3}+10 I_{4}+I_{5},
$$

where, for $i \in\{1, \ldots, 5\}$,

$$
I_{i}=\mathbb{E} \sum_{\left(x_{1}, \ldots, x_{i}\right) \in \mathcal{X}_{n-1-|\mathcal{A}|, \neq}^{i}} \mathbf{1}_{\left\{D_{\left(y, M_{y}\right)} \xi_{n}\left(x_{j}, \mathcal{X}_{n, \mathcal{A}}\right) \neq 0, j=1, \ldots, i\right\}}\left|D_{\left(y, M_{y}\right)} \xi_{n}\left(x_{1}, \mathcal{X}_{n, \mathcal{A}}\right)\right|^{4+\varepsilon} .
$$


It follows from Hölder's inequality that $I_{i}$ equals

$$
\begin{aligned}
& \left.\frac{(n-1-|\mathcal{A}|) !}{(n}-1-|\mathcal{A}|-i\right) ! \\
& \quad \times \int_{\widehat{\mathbb{X}} i} \mathbb{E} \mathbf{1}_{\left\{D_{\left(y, M_{y}\right)} \xi_{n}\left(x_{j}, \mathcal{X}_{n-i, \mathcal{A}} \cup\left\{x_{1}, \ldots, x_{i}\right\}\right) \neq 0, j=1, \ldots, i\right\}} \\
& \quad \times\left|D_{\left(y, M_{y}\right)} \xi_{n}\left(x_{1}, \mathcal{X}_{n-i, \mathcal{A}} \cup\left\{x_{1}, \ldots, x_{i}\right\}\right)\right|^{4+\varepsilon} \widehat{\mathbb{Q}}^{i}\left(\mathrm{~d}\left(x_{1}, \ldots, x_{i}\right)\right) \\
& \leq n^{i} \int_{\mathbb{X} i} \prod_{j=1}^{i} \mathbb{P}\left(D_{\left(y, M_{y}\right)} \xi_{n}\left(\left(x_{j}, M_{x_{j}}\right), \mathcal{X}_{n-i, \mathcal{A}} \cup\left\{\left(x_{1}, M_{x_{1}}\right), \ldots,\left(x_{i}, M_{x_{i}}\right)\right\}\right) \neq 0\right)^{\frac{p-\varepsilon}{4 i+p i}} \\
& \quad \times\left(\mathbb{E}\left|D_{\left(y, M_{y}\right)} \xi_{n}\left(\left(x_{1}, M_{x_{1}}\right), \mathcal{X}_{n-i, \mathcal{A}} \cup\left\{\left(x_{1}, M_{x_{1}}\right), \ldots,\left(x_{i}, M_{x_{i}}\right)\right\}\right)\right|^{4+p}\right)^{\frac{4+\varepsilon}{4+p}} \\
& \quad \times \mathbb{Q}^{i}\left(\mathrm{~d}\left(x_{1}, \ldots, x_{i}\right)\right) .
\end{aligned}
$$

Combining this with Lemma 5.3, (2.5) and Lemma 5.4(b) leads to

$$
\begin{aligned}
I_{i} & \leq 2^{4+\varepsilon} C_{p}^{\frac{4+\varepsilon}{4+p}} n^{i} \int_{\mathbb{X}^{i}} C^{\frac{p-\varepsilon}{4+p}} \prod_{j=1}^{i} \exp \left(-\frac{c(p-\varepsilon)}{4 i+p i} \mathrm{~d}_{n}\left(x_{j}, y\right)^{\alpha}\right) \mathbb{Q}^{i}\left(\mathrm{~d}\left(x_{1}, \ldots, x_{i}\right)\right) \\
& =2^{4+\varepsilon} C_{p}^{\frac{4+\varepsilon}{4+p}}\left(n C^{\frac{p-\varepsilon}{4 i+p i}} \int_{\mathbb{X}} \exp \left(-\frac{c(p-\varepsilon)}{4 i+p i} \mathrm{~d}_{n}(x, y)^{\alpha}\right) \mathbb{Q}(\mathrm{d} x)\right)^{i} .
\end{aligned}
$$

Now (5.2) yields that the integrals on the right-hand side are uniformly bounded.

LEMMA 5.7. (a) For $x, z \in \mathbb{X}$ and $s \geq 1$,

$$
\begin{aligned}
& \mathbb{P}\left(D_{\left(x, M_{x}\right)} \xi_{s}\left(\left(z, M_{z}\right), \mathcal{P}_{s} \cup\left\{\left(z, M_{z}\right)\right\}\right) \neq 0\right) \\
& \leq 2 C \exp \left(-c \max \left\{\mathrm{d}_{s}(x, z), \mathrm{d}_{s}(z, K)\right\}^{\alpha}\right) .
\end{aligned}
$$

(b) For $x_{1}, x_{2}, z \in \mathbb{X}$ and $s \geq 1$,

$$
\begin{aligned}
& \mathbb{P}\left(D_{\left(x_{1}, M_{x_{1}}\right),\left(x_{2}, M_{x_{2}}\right)}^{2} \xi_{s}\left(\left(z, M_{z}\right), \mathcal{P}_{s} \cup\left\{\left(z, M_{z}\right)\right\}\right) \neq 0\right) \\
& \quad \leq 4 C \exp \left(-c \max \left\{\mathrm{d}_{s}\left(x_{1}, z\right), \mathrm{d}_{s}\left(x_{2}, z\right), \mathrm{d}_{s}(z, K)\right\}^{\alpha}\right) .
\end{aligned}
$$

PROOF. We prove part (b). By Lemma 5.3, the event

$$
D_{\left(x_{1}, M_{x_{1}}\right),\left(x_{2}, M_{x_{2}}\right)}^{2} \xi_{s}\left(\left(z, M_{z}\right), \mathcal{P}_{s} \cup\left\{\left(z, M_{z}\right)\right\}\right) \neq 0
$$

is a subset of the event that the points $x_{1}, x_{2}$ belong to the ball centered at $z$ with radius $R_{s}\left(\left(z, M_{z}\right), \mathcal{P}_{s} \cup\left\{\left(z, M_{z}\right)\right\}\right)$, that is, $R_{s}\left(\left(z, M_{z}\right), \mathcal{P}_{s} \cup\left\{\left(z, M_{z}\right)\right\}\right) \geq$ $\max \left\{\mathrm{d}\left(x_{1}, z\right), \mathrm{d}\left(x_{2}, z\right)\right\}$. By $(2.4)$, this gives

$$
\begin{gathered}
\mathbb{P}\left(D_{\left(x_{1}, M_{x_{1}}\right),\left(x_{2}, M_{x_{2}}\right)}^{2} \xi_{s}\left(\left(z, M_{z}\right), \mathcal{P}_{s} \cup\left\{\left(z, M_{z}\right)\right\}\right) \neq 0\right) \\
\leq C \exp \left(-c \max \left\{\mathrm{d}_{s}\left(x_{1}, z\right), \mathrm{d}_{s}\left(x_{2}, z\right)\right\}^{\alpha}\right) .
\end{gathered}
$$


By (2.8), we also have

$$
\mathbb{P}\left(D_{\left(x_{1}, M_{x_{1}}\right),\left(x_{2}, M_{x_{2}}\right)}^{2} \xi_{s}\left(\left(z, M_{z}\right), \mathcal{P}_{s} \cup\left\{\left(z, M_{z}\right)\right\}\right) \neq 0\right) \leq 4 C \exp \left(-c \mathrm{~d}_{s}(z, K)^{\alpha}\right) .
$$

This gives the proof of part (b). Part (a) is proven in a similar way.

LEMMA 5.8. (a) For $x, z \in \mathbb{X}$ and $n \geq 9$,

$$
\begin{aligned}
& \sup _{\mathcal{A} \subset \mathbb{X},|\mathcal{A}| \leq 1} \mathbb{P}\left(D_{\left(x, M_{x}\right)} \xi_{n}\left(\left(z, M_{z}\right), \mathcal{X}_{n-2-|\mathcal{A}|} \cup\left\{\left(z, M_{z}\right)\right\} \cup\left(\mathcal{A}, M_{\mathcal{A}}\right)\right) \neq 0\right) \\
& \quad \leq 2 C \exp \left(-c \max \left\{\mathrm{d}_{n}(x, z), \mathrm{d}_{n}(z, K)\right\}^{\alpha}\right)
\end{aligned}
$$

(b) For $x_{1}, x_{2}, z \in \mathbb{X}$ and $n \geq 9$,

$$
\begin{aligned}
& \sup _{\mathcal{A} \subset \mathbb{X},|\mathcal{A}| \leq 1} \mathbb{P}\left(D_{\left(x_{1}, M_{x_{1}}\right),\left(x_{2}, M_{x_{2}}\right)}^{2} \xi_{n}\left(\left(z, M_{z}\right), \mathcal{X}_{n-3-|\mathcal{A}|} \cup\left\{\left(z, M_{z}\right)\right\} \cup\left(\mathcal{A}, M_{\mathcal{A}}\right)\right) \neq 0\right) \\
& \quad \leq 4 C \exp \left(-c \max \left\{\mathrm{d}_{n}\left(x_{1}, z\right), \mathrm{d}_{n}\left(x_{2}, z\right), \mathrm{d}_{n}(z, K)\right\}^{\alpha}\right) .
\end{aligned}
$$

PROOF. By Lemma 5.3 and (2.5) together with similar arguments as in the proof of Lemma 5.7, we obtain

$$
\begin{aligned}
& \mathbb{P}\left(D_{\left(x_{1}, M_{x_{1}}\right),\left(x_{2}, M_{x_{2}}\right)}^{2} \xi_{n}\left(\left(z, M_{z}\right), \mathcal{X}_{n-3-|\mathcal{A}|} \cup\left\{\left(z, M_{z}\right)\right\} \cup\left(\mathcal{A}, M_{\mathcal{A}}\right)\right) \neq 0\right) \\
& \quad \leq \mathbb{P}\left(R_{n}\left(\left(z, M_{z}\right), \mathcal{X}_{n-8} \cup\left\{\left(z, M_{z}\right)\right\}\right) \geq \max \left\{\mathrm{d}_{s}\left(x_{1}, z\right), \mathrm{d}_{s}\left(x_{2}, z\right)\right\}\right) \\
& \quad \leq C \exp \left(-c \max \left\{\mathrm{d}_{s}\left(x_{1}, z\right), \mathrm{d}_{s}\left(x_{2}, z\right)\right\}^{\alpha}\right) .
\end{aligned}
$$

It follows from (2.9) that

$$
\begin{aligned}
& \mathbb{P}\left(D_{\left(x_{1}, M_{x_{1}}\right),\left(x_{2}, M_{x_{2}}\right)}^{2} \xi_{n}\left(\left(z, M_{z}\right), \mathcal{X}_{n-3-|\mathcal{A}|} \cup\left\{\left(z, M_{z}\right)\right\} \cup\left(\mathcal{A}, M_{\mathcal{A}}\right)\right) \neq 0\right) \\
& \quad \leq 4 C \exp \left(-c \mathrm{~d}_{s}(z, K)^{\alpha}\right),
\end{aligned}
$$

which completes the proof of part (b). Part (a) is proven similarly.

LEMMA 5.9. (a) Let $\beta \in(0, \infty)$ be fixed. Then there is a constant $C_{\beta} \in(0, \infty)$ such that

$$
s \int_{\mathbb{X}} \mathbb{P}\left(D_{\left(x_{1}, M_{x_{1}}\right),\left(x_{2}, M_{x_{2}}\right)}^{2} h_{s}\left(\mathcal{P}_{s}\right) \neq 0\right)^{\beta} \mathbb{Q}\left(\mathrm{d} x_{2}\right) \leq C_{\beta} \exp \left(-c \beta \mathrm{d}_{s}\left(x_{1}, K\right) / 4^{\alpha+1}\right)
$$

for all $x_{1} \in \mathbb{X}$ and $s \in[1, \infty)$.

(b) Let $\beta \in(0, \infty)$ be fixed. Then there is a constant $C_{\beta} \in(0, \infty)$ such that

$$
\begin{aligned}
& n \int_{\mathbb{X}} \sup _{\mathcal{A} \subset \mathbb{X},|\mathcal{A}| \leq 1} \mathbb{P}\left(D_{\left(x_{1}, M_{x_{1}}\right),\left(x_{2}, M_{x_{2}}\right)}^{2} h_{n}\left(\mathcal{X}_{n-2-|\mathcal{A}|} \cup\left(\mathcal{A}, M_{\mathcal{A}}\right)\right) \neq 0\right)^{\beta} \mathbb{Q}\left(\mathrm{d} x_{2}\right) \\
& \quad \leq C_{\beta} \exp \left(-c \beta \mathrm{d}_{s}\left(x_{1}, K\right) / 4^{\alpha+1}\right)
\end{aligned}
$$

for all $x_{1} \in \mathbb{X}$ and $n \geq 9$. 
PROOF. By Lemma 5.2, we have

$$
\begin{aligned}
D_{\left(x_{1}, M_{x_{1}}\right),\left(x_{2}, M_{x_{2}}\right)}^{2} h_{s}\left(\mathcal{P}_{s}\right)= & D_{\left(x_{1}, M_{x_{1}}\right)} \xi_{s}\left(\left(x_{2}, M_{x_{2}}\right), \mathcal{P}_{s} \cup\left\{\left(x_{2}, M_{x_{2}}\right)\right\}\right) \\
& +D_{\left(x_{2}, M_{x_{2}}\right)} \xi_{s}\left(\left(x_{1}, M_{x_{1}}\right), \mathcal{P}_{s} \cup\left\{\left(x_{1}, M_{x_{1}}\right)\right\}\right) \\
& +\sum_{z \in \mathcal{P}_{s}} D_{\left(x_{1}, M_{x_{1}}\right),\left(x_{2}, M_{x_{2}}\right)}^{2} \xi_{s}\left(z, \mathcal{P}_{s}\right)
\end{aligned}
$$

so that the Slivnyak-Mecke formula leads to

$$
\begin{aligned}
& \mathbb{P}\left(D_{\left(x_{1}, M_{x_{1}}\right),\left(x_{2}, M_{x_{2}}\right)}^{2} h_{s}\left(\mathcal{P}_{s}\right) \neq 0\right) \\
& \leq \mathbb{P}\left(D_{\left(x_{1}, M_{x_{1}}\right)} \xi_{s}\left(\left(x_{2}, M_{x_{2}}\right), \mathcal{P}_{s} \cup\left\{\left(x_{2}, M_{x_{2}}\right)\right\}\right) \neq 0\right) \\
&+\mathbb{P}\left(D_{\left(x_{2}, M_{x_{2}}\right)} \xi_{s}\left(\left(x_{1}, M_{x_{1}}\right), \mathcal{P}_{s} \cup\left\{\left(x_{1}, M_{x_{1}}\right)\right\}\right) \neq 0\right) \\
&+\underbrace{s \int_{\mathbb{X}} \mathbb{P}\left(D_{\left(x_{1}, M_{x_{1}}\right),\left(x_{2}, M_{x_{2}}\right)}^{2} \xi_{s}\left(\left(z, M_{z}\right), \mathcal{P}_{s} \cup\left\{\left(z, M_{z}\right)\right\}\right) \neq 0\right) \mathbb{Q}(\mathrm{d} z) .}_{=: T_{x_{1}, x_{2}, s}}
\end{aligned}
$$

Here, we use part (a) of Lemma 5.7 to bound each of the first two terms on the right-hand side. We may bound the first term by

$$
\begin{array}{r}
\mathbb{P}\left(D_{\left(x_{1}, M_{x_{1}}\right)} \xi_{s}\left(\left(x_{2}, M_{x_{2}}\right), \mathcal{P}_{s} \cup\left\{\left(x_{2}, M_{x_{2}}\right)\right\}\right) \neq 0\right) \\
\leq 2 C \exp \left(-c \max \left\{\mathrm{d}_{s}\left(x_{1}, x_{2}\right), \mathrm{d}_{s}\left(x_{2}, K\right)\right\}^{\alpha}\right) .
\end{array}
$$

Observing that $\mathrm{d}_{s}\left(x_{1}, K\right) \leq 2 \max \left\{\mathrm{d}_{s}\left(x_{2}, K\right), \mathrm{d}_{s}\left(x_{1}, x_{2}\right)\right\}$, we obtain

$$
\begin{aligned}
& \mathbb{P}\left(D_{\left(x_{1}, M_{x_{1}}\right)} \xi_{s}\left(\left(x_{2}, M_{x_{2}}\right), \mathcal{P}_{s} \cup\left\{\left(x_{2}, M_{x_{2}}\right)\right\}\right) \neq 0\right) \\
& \quad \leq 2 C \exp \left(-c \max \left\{\mathrm{d}_{s}\left(x_{1}, x_{2}\right), \mathrm{d}_{s}\left(x_{1}, K\right), \mathrm{d}_{s}\left(x_{2}, K\right)\right\}^{\alpha} / 2^{\alpha}\right) .
\end{aligned}
$$

We bound $\mathbb{P}\left(D_{\left(x_{2}, M_{x_{2}}\right)} \xi_{s}\left(\left(x_{1}, M_{x_{1}}\right), \mathcal{P}_{s} \cup\left\{\left(x_{1}, M_{x_{1}}\right)\right\}\right) \neq 0\right)$ in the same way. It follows from part (b) of Lemma 5.7 that

$$
T_{x_{1}, x_{2}, s} \leq 4 C s \int_{\mathbb{X}} \exp \left(-c \max \left\{\mathrm{d}_{s}\left(x_{1}, z\right), \mathrm{d}_{s}\left(x_{2}, z\right), \mathrm{d}_{s}(z, K)\right\}^{\alpha}\right) \mathbb{Q}(\mathrm{d} z) .
$$

Assume that $\mathrm{d}_{s}\left(x_{1}, K\right) \geq \mathrm{d}_{s}\left(x_{2}, K\right)$ [the reasoning is similar if $\mathrm{d}_{s}\left(x_{2}, K\right) \geq$ $\left.\mathrm{d}_{s}\left(x_{1}, K\right)\right]$ and let $r=\max \left\{\mathrm{d}\left(x_{1}, K\right), \mathrm{d}\left(x_{1}, x_{2}\right)\right\} / 2$. For any $z \in B\left(x_{1}, r\right)$, the triangle inequality leads to $\max \left\{\mathrm{d}\left(z, x_{2}\right), \mathrm{d}(z, K)\right\} \geq r$. This implies that

$$
\begin{aligned}
T_{x_{1}, x_{2}, s} \leq & 4 C s \int_{B\left(x_{1}, r\right)} \exp (-c \underbrace{\max \left\{\mathrm{d}_{s}\left(z, x_{2}\right), \mathrm{d}_{s}(z, K)\right\}^{\alpha}}_{\geq\left(s^{1 / \gamma} r\right)^{\alpha}}) \mathbb{Q}(\mathrm{d} z) \\
& +4 C s \int_{\mathbb{X} \backslash B\left(x_{1}, r\right)} \exp \left(-c \mathrm{~d}_{s}\left(x_{1}, z\right)^{\alpha}\right) \mathbb{Q}(\mathrm{d} z) .
\end{aligned}
$$


Recalling from (5.1) that $\mathbb{Q}\left(B\left(x_{1}, r\right)\right) \leq \kappa r^{\gamma}$, we have

$$
\begin{aligned}
4 C s \int_{B\left(x_{1}, r\right)} \exp \left(-c\left(s^{1 / \gamma} r\right)^{\alpha}\right) \mathbb{Q}(\mathrm{d} z) & \leq 4 C s \kappa r^{\gamma} \exp \left(-c\left(s^{1 / \gamma} r\right)^{\alpha}\right) \\
& \leq C_{1} \exp \left(-c\left(s^{1 / \gamma} r\right)^{\alpha} / 2\right)
\end{aligned}
$$

with a constant $C_{1} \in(0, \infty)$ only depending on $C, c, \gamma$ and $\alpha$. On the other hand, (5.2) yields

$$
4 C s \int_{\mathbb{X} \backslash B\left(x_{1}, r\right)} \exp \left(-c \mathrm{~d}_{s}\left(x_{1}, z\right)^{\alpha}\right) \mathbb{Q}(\mathrm{d} z) \leq C_{2} \exp \left(-c\left(s^{1 / \gamma} r\right)^{\alpha} / 2\right)
$$

with a constant $C_{2} \in(0, \infty)$ only depending on $C, c, \gamma$ and $\alpha$. Hence, we obtain

$$
T_{x_{1}, x_{2}, s} \leq\left(C_{1}+C_{2}\right) \exp \left(-c \max \left\{\mathrm{d}_{s}\left(x_{1}, K\right), \mathrm{d}_{s}\left(x_{2}, K\right), \mathrm{d}_{s}\left(x_{1}, x_{2}\right)\right\}^{\alpha} / 2^{\alpha+1}\right)
$$

and

$$
\begin{aligned}
& \mathbb{P}\left(D_{\left(x_{1}, M_{x_{1}}\right),\left(x_{2}, M_{x_{2}}\right)}^{2} h_{s}\left(\mathcal{P}_{s}\right) \neq 0\right) \\
& \quad \leq C_{3} \exp \left(-c \max \left\{\mathrm{d}_{s}\left(x_{1}, K\right), \mathrm{d}_{s}\left(x_{2}, K\right), \mathrm{d}_{s}\left(x_{1}, x_{2}\right)\right\}^{\alpha} / 2^{\alpha+1}\right)
\end{aligned}
$$

with $C_{3}:=C_{1}+C_{2}+4 C$. Consequently, we have

$$
\begin{aligned}
s \int_{\mathbb{X}} \mathbb{P} & \left(D_{\left(x_{1}, M_{x_{1}}\right),\left(x_{2}, M_{x_{2}}\right)}^{2} h_{s}\left(\mathcal{P}_{s}\right) \neq 0\right)^{\beta} \mathbb{Q}\left(\mathrm{d} x_{2}\right) \\
\leq & C_{3}^{\beta} s \int_{B\left(x_{1}, \mathrm{~d}\left(x_{1}, K\right) / 2\right)} \exp \left(-c \beta \mathrm{d}_{s}\left(x_{2}, K\right)^{\alpha} / 2^{\alpha+1}\right) \mathbb{Q}\left(\mathrm{d} x_{2}\right) \\
& +C_{3}^{\beta} s \int_{\mathbb{X} \backslash B\left(x_{1}, \mathrm{~d}\left(x_{1}, K\right) / 2\right)} \exp \left(-c \beta \mathrm{d}_{s}\left(x_{2}, x_{1}\right)^{\alpha} / 2^{\alpha+1}\right) \mathbb{Q}\left(\mathrm{d} x_{2}\right) .
\end{aligned}
$$

Using the same arguments as above, the right-hand side can be bounded by

$$
C_{\beta} \exp \left(-c \beta \mathrm{d}_{s}\left(K, x_{1}\right)^{\alpha} / 4^{\alpha+1}\right)
$$

with a constant $C_{\beta} \in(0, \infty)$ only depending on $\beta, C_{3}, c, \gamma$ and $\alpha$. This completes the proof of (a).

Similar arguments show for the binomial case that

$$
\begin{aligned}
& \sup _{\mathcal{A} \subset \mathbb{X},|\mathcal{A}| \leq 1} \mathbb{P}\left(D_{\left(x_{1}, M_{x_{1}}\right),\left(x_{2}, M_{x_{2}}\right)}^{2} h_{n}\left(\mathcal{X}_{n-2-|\mathcal{A}|} \cup\left(\mathcal{A}, M_{\mathcal{A}}\right)\right) \neq 0\right) \\
& \leq \sup _{\mathcal{A} \subset \mathbb{X},|\mathcal{A}| \leq 1} \mathbb{P}\left(D_{\left(x_{1}, M_{x_{1}}\right)} \xi_{n}\left(\left(x_{2}, M_{x_{2}}\right), \mathcal{X}_{n-2-|\mathcal{A}|} \cup\left\{\left(x_{2}, M_{x_{2}}\right)\right\} \cup\left(\mathcal{A}, M_{\mathcal{A}}\right)\right) \neq 0\right) \\
& \quad+\sup _{\mathcal{A} \subset \mathbb{X},|\mathcal{A}| \leq 1} \mathbb{P}\left(D_{\left(x_{2}, M_{x_{2}}\right)} \xi_{n}\left(\left(x_{1}, M_{x_{1}}\right), \mathcal{X}_{n-2-|\mathcal{A}|} \cup\left\{\left(x_{1}, M_{x_{1}}\right)\right\} \cup\left(\mathcal{A}, M_{\mathcal{A}}\right)\right) \neq 0\right) \\
& +n \int_{\mathbb{X}} \sup _{\mathcal{A} \subset \mathbb{X},|\mathcal{A}| \leq 1} \mathbb{P}\left(D _ { ( x _ { 1 } , M _ { x _ { 1 } } ) , ( x _ { 2 } , M _ { x _ { 2 } } ) } ^ { 2 } \xi _ { n } \left(\left(z, M_{z}\right), \mathcal{X}_{n-3-|\mathcal{A}|}\right.\right. \\
& \left.\left.\quad \cup\left\{\left(z, M_{z}\right)\right\} \cup\left(\mathcal{A}, M_{\mathcal{A}}\right)\right) \neq 0\right) \mathbb{Q}(\mathrm{d} z) \\
& +\sup _{z \in \mathbb{X}} \mathbb{P}\left(D_{\left(x_{1}, M_{x_{1}}\right),\left(x_{2}, M_{x_{2}}\right)}^{2} \xi_{n}\left(\left(z, M_{z}\right), \mathcal{X}_{n-3} \cup\left\{\left(z, M_{z}\right)\right\}\right) \neq 0\right) .
\end{aligned}
$$


Now similar computations as for the Poisson case conclude the proof of part (b).

For $\alpha, \tau \geq 0$, put

$$
I_{K, s}(\alpha, \tau):=s \int_{\mathbb{X}} \exp \left(-\tau \mathrm{d}_{s}(x, K)^{\alpha}\right) \mathbb{Q}(\mathrm{d} x), \quad s \geq 1 .
$$

LEMmA 5.10. Let $\beta \in(0, \infty)$ be fixed. There is a constant $\tilde{C}_{\beta} \in(0, \infty)$ such that for all $s \geq 1$ we have

$$
\begin{aligned}
& s \int_{\mathbb{X}}\left(s \int_{\mathbb{X}} \mathbb{P}\left(D_{\left(x_{1}, M_{x_{1}}\right),\left(x_{2}, M_{x_{2}}\right)}^{2} h_{s}\left(\mathcal{P}_{s}\right) \neq 0\right)^{\beta} \mathbb{Q}\left(\mathrm{d} x_{2}\right)\right)^{2} \mathbb{Q}\left(\mathrm{d} x_{1}\right) \\
& \quad \leq \tilde{C}_{\beta} I_{K, s}\left(\alpha, c \beta / 2^{2 \alpha+1}\right), \\
& s^{2} \int_{\mathbb{X}^{2}} \mathbb{P}\left(D_{\left(x_{1}, M_{x_{1}}\right),\left(x_{2}, M_{x_{2}}\right)}^{2} h_{s}\left(\mathcal{P}_{s}\right) \neq 0\right)^{\beta} \mathbb{Q}^{2}\left(\mathrm{~d}\left(x_{1}, x_{2}\right)\right) \\
& \quad \leq \tilde{C}_{\beta} I_{K, s}\left(\alpha, c \beta / 4^{\alpha+1}\right)
\end{aligned}
$$

and

$$
s \int_{\mathbb{X}} \mathbb{P}\left(D_{\left(x, M_{x}\right)} h_{s}\left(\mathcal{P}_{s}\right) \neq 0\right)^{\beta} \mathbb{Q}(\mathrm{d} x) \leq \tilde{C}_{\beta} I_{K, s}\left(\alpha, c \beta / 2^{\alpha+1}\right)
$$

PROOF. By Lemma 5.9(a), the integrals in (5.6) and (5.7) are bounded by

$$
C_{\beta}^{2} s \int_{\mathbb{X}} \exp \left(-c \beta \mathrm{d}_{s}\left(x_{1}, K\right)^{\alpha} / 2^{2 \alpha+1}\right) \mathbb{Q}\left(\mathrm{d} x_{1}\right)=C_{\beta}^{2} I_{k, s}\left(\alpha, c \beta / 2^{2 \alpha+1}\right)
$$

and

$$
C_{\beta} s \int_{\mathbb{X}} \exp \left(-c \beta \mathrm{d}_{s}\left(x_{1}, K\right)^{\alpha} / 4^{\alpha+1}\right) \mathbb{Q}\left(\mathrm{d} x_{1}\right)=C_{\beta} I_{k, s}\left(\alpha, c \beta / 4^{\alpha+1}\right),
$$

respectively. In order to derive the bound in (5.8), we compute $\mathbb{P}\left(D_{\left(x, M_{x}\right)} h_{S}\left(\mathcal{P}_{S}\right) \neq\right.$ 0) as follows. By Lemma 5.2 and the Slivnyack-Mecke formula, we obtain that

$$
\begin{aligned}
& \mathbb{P}\left(D_{\left(x, M_{x}\right)} h_{s}\left(\mathcal{P}_{s}\right) \neq 0\right) \\
& \leq \mathbb{P}\left(\xi_{s}\left(\left(x, M_{x}\right), \mathcal{P}_{s} \cup\left\{\left(x, M_{x}\right)\right\}\right) \neq 0\right)+\mathbb{E} \sum_{z \in \mathcal{P}_{s}} \mathbf{1}_{\left\{D_{\left(x, M_{x}\right)} \xi_{s}\left(z, \mathcal{P}_{s}\right) \neq 0\right\}} \\
&= \mathbb{P}\left(\xi_{s}\left(\left(x, M_{x}\right), \mathcal{P}_{s} \cup\left\{\left(x, M_{x}\right)\right\}\right) \neq 0\right) \\
&+s \int_{\mathbb{X}} \mathbb{P}\left(D_{\left(x, M_{x}\right)} \xi_{s}\left(\left(z, M_{z}\right), \mathcal{P}_{s} \cup\left\{\left(z, M_{z}\right)\right\}\right) \neq 0\right) \mathbb{Q}(\mathrm{d} z) .
\end{aligned}
$$

By (2.8) and Lemma 5.7(a), we obtain that for all $x \in \mathbb{X}$ and $s \geq 1$,

$$
\begin{aligned}
\mathbb{P}\left(D_{\left(x, M_{x}\right)} h_{s}\left(\mathcal{P}_{s}\right) \neq 0\right) \leq & C \exp \left(-c \mathrm{~d}_{s}(x, K)^{\alpha}\right) \\
& +2 C s \int_{\mathbb{X}} \exp \left(-c \max \left\{\mathrm{d}_{s}(x, z), \mathrm{d}_{s}(z, K)\right\}^{\alpha}\right) \mathbb{Q}(\mathrm{d} z) .
\end{aligned}
$$


Letting $r:=\mathrm{d}(x, K) / 2$, partitioning $\mathbb{X}$ into the union of $\mathbb{X} \backslash B(x, r)$ and $B(x, r)$, and following the discussion in the proof of Lemma 5.9, we obtain

$$
2 C s \int_{\mathbb{X}} \exp \left(-c \max \left\{\mathrm{d}_{s}(x, z), \mathrm{d}_{s}(z, K)\right\}^{\alpha}\right) \mathbb{Q}(\mathrm{d} z) \leq C_{1} \exp \left(-c \mathrm{~d}_{s}(x, K)^{\alpha} / 2^{\alpha+1}\right)
$$

with a constant $C_{1} \in(0, \infty)$. Consequently, for all $x \in \mathbb{X}$ and $s \geq 1$ we have

$$
\mathbb{P}\left(D_{\left(x, M_{x}\right)} h_{s}\left(\mathcal{P}_{s}\right) \neq 0\right) \leq\left(C+C_{1}\right) \exp \left(-c \mathrm{~d}_{s}(x, K)^{\alpha} / 2^{\alpha+1}\right)
$$

and

$$
s \int_{\mathbb{X}} \mathbb{P}\left(D_{\left(x, M_{x}\right)} h_{s}\left(\mathcal{P}_{s}\right) \neq 0\right)^{\beta} \mathbb{Q}(\mathrm{d} x) \leq\left(C+C_{1}\right) I_{K, s}\left(\alpha, c \beta / 2^{\alpha+1}\right),
$$

which was to be shown.

LEMMA 5.11. Let $\beta \in(0, \infty)$ be fixed. There is a constant $\tilde{C}_{\beta} \in(0, \infty)$ such that for all $n \geq 9$ we have

$$
\begin{aligned}
& n \int_{\mathbb{X}}\left(n \int_{\mathbb{X}} \sup _{\mathcal{A} \subset \mathbb{X},|\mathcal{A}| \leq 1} \mathbb{P}\left(D_{\left(x_{1}, M_{x_{1}}\right),\left(x_{2}, M_{x_{2}}\right)}^{2} h_{n}\left(\mathcal{X}_{n-2-|\mathcal{A}|} \cup\left(\mathcal{A}, M_{\mathcal{A}}\right)\right) \neq 0\right)^{\beta} \mathbb{Q}\left(\mathrm{d} x_{2}\right)\right)^{2} \\
& \quad \times \mathbb{Q}\left(\mathrm{d} x_{1}\right) \\
& \quad \leq \tilde{C}_{\beta} I_{K, n}\left(\alpha, c \beta / 2^{2 \alpha+1}\right), \\
& n^{2} \int_{\mathbb{X}^{2}} \sup _{\mathcal{A} \subset \mathbb{X},|\mathcal{A}| \leq 1} \mathbb{P}\left(D_{\left(x_{1}, M_{x_{1}}\right),\left(x_{2}, M_{x_{2}}\right)}^{2} h_{n}\left(\mathcal{X}_{n-2-|\mathcal{A}|} \cup\left(\mathcal{A}, M_{\mathcal{A}}\right)\right) \neq 0\right)^{\beta} \mathbb{Q}^{2}\left(\mathrm{~d}\left(x_{1}, x_{2}\right)\right) \\
& \quad \leq \tilde{C}_{\beta} I_{K, n}\left(\alpha, c \beta / 4^{\alpha+1}\right)
\end{aligned}
$$

and

$$
n \int_{\mathbb{X}} \mathbb{P}\left(D_{\left(x, M_{x}\right)} h_{n}\left(\mathcal{X}_{n-1}\right) \neq 0\right)^{\beta} \mathbb{Q}(\mathrm{d} x) \leq \tilde{C}_{\beta} I_{K, n}\left(\alpha, c \beta / 2^{\alpha+1}\right)
$$

ProOF. The bounds in (5.9) and (5.11) follow immediately from Lemma 5.9(b) and the definition of $I_{K, n}(\alpha, \tau)$. By Lemma 5.2, we obtain that, for $x \in \mathbb{X}$,

$$
\begin{aligned}
& \mathbb{P}\left(D_{\left(x, M_{x}\right)} h_{n}\left(\mathcal{X}_{n-1}\right) \neq 0\right) \\
& \leq \mathbb{P}\left(\xi_{n}\left(\left(x, M_{x}\right), \mathcal{X}_{n-1} \cup\left\{\left(x, M_{x}\right)\right\}\right) \neq 0\right) \\
&+\mathbb{E} \sum_{z \in \mathcal{X}_{n-1}} \mathbf{1}_{\left\{D_{\left(x, M_{x}\right)} \xi_{n}\left(z, \mathcal{X}_{n-1} \cup\{z\}\right) \neq 0\right\}} \\
& \leq \mathbb{P}\left(\xi_{n}\left(\left(x, M_{x}\right), \mathcal{X}_{n-1} \cup\left\{\left(x, M_{x}\right)\right\}\right) \neq 0\right) \\
&+n \int_{\mathbb{X}} \mathbb{P}\left(D_{\left(x, M_{x}\right)} \xi_{n}\left(\left(z, M_{z}\right), \mathcal{X}_{n-2} \cup\left\{\left(z, M_{z}\right)\right\}\right) \neq 0\right) \mathbb{Q}(\mathrm{d} z) .
\end{aligned}
$$


Combining the bound from Lemma 5.8(a) with the computations from the proof of Lemma 5.10 and (2.9), we see that there is a constant $C_{1} \in(0, \infty)$ such that for all $x \in \mathbb{X}$ and $s \geq 1$ we have

$$
\mathbb{P}\left(D_{\left(x, M_{x}\right)} h_{n}\left(\mathcal{X}_{n-1}\right) \neq 0\right) \leq C_{1} \exp \left(-c \mathrm{~d}_{s}(x, K)^{\alpha} / 2^{\alpha+1}\right) .
$$

Now (5.10) follows from the definition of $I_{K, n}(\alpha, \tau)$.

Proof of TheOrem 2.1. We start with the proof of the Poisson case (2.11). It follows from Lemma 5.5 that the condition (4.1) with the exponent $4+p / 2$ in Theorem 4.1 is satisfied for all $s \geq 1$ with the constant $C_{p / 2}$. In the following, we use the abbreviation

$$
I_{K, s}=I_{K, s}\left(\alpha, c p /\left(36 \cdot 4^{\alpha+1}\right)\right) .
$$

Together with Lemma 5.10 (with $\beta=p / 36$ ), it follows from Theorem 4.1 that there is a constant $\tilde{C} \in(0, \infty)$ depending on $\tilde{C}_{p / 36}, C_{p / 2}$ and $p$ such that

$$
d_{K}\left(\frac{H_{s}-\mathbb{E} H_{s}}{\sqrt{\operatorname{Var} H_{s}}}, N\right) \leq \tilde{C}\left(\frac{\sqrt{I_{K, s}}}{\operatorname{Var} H_{s}}+\frac{I_{K, s}}{\left(\operatorname{Var} H_{s}\right)^{3 / 2}}+\frac{I_{K, s}^{5 / 4}+I_{K, s}^{3 / 2}}{\left(\operatorname{Var} H_{s}\right)^{2}}\right),
$$

which completes the proof of the Poisson case.

For the binomial case (2.12), it follows from Lemma 5.6 that the condition (4.3) in Theorem 4.2 is satisfied with the exponent $4+p / 2$ for all $n \geq 9$ with the same constant $C_{p / 2} \geq 1$. Using the abbreviation

$$
I_{K, n}=I_{K, n}\left(\alpha, c p /\left(18 \cdot 4^{\alpha+1}\right)\right),
$$

we obtain from Lemma 5.11 (with $\beta=p / 18$ ) and Theorem 4.2 that there is a constant $\tilde{C} \in(0, \infty)$ depending on $\tilde{C}_{p / 18}, C_{p / 2}$ and $p$ such that

$$
d_{K}\left(\frac{H_{n}^{\prime}-\mathbb{E} H_{n}^{\prime}}{\sqrt{\operatorname{Var} H_{n}^{\prime}}}, N\right) \leq \tilde{C}\left(\frac{\sqrt{I_{K, n}}}{\operatorname{Var} H_{n}^{\prime}}+\frac{I_{K, n}}{\left(\operatorname{Var} H_{n}^{\prime}\right)^{3 / 2}}+\frac{I_{K, n}+\left(I_{K, n}\right)^{3 / 2}}{\left(\operatorname{Var} H_{n}^{\prime}\right)^{2}}\right),
$$

which completes the proof.

Before giving the proof of Theorem 2.3, we require a lemma. We thank Steffen Winter for discussions concerning the proof. For $K \subset \mathbb{R}^{d}$, recall that $K_{r}:=\{y \in$ $\left.\mathbb{R}^{d}: \mathrm{d}(y, K) \leq r\right\}$ and that $\overline{\mathcal{M}}^{d-1}(K)$ is defined at (2.16).

LEMMA 5.12. If $K$ is either a full-dimensional compact subset of $\mathbb{R}^{d}$ with $\overline{\mathcal{M}}^{d-1}(\partial K)<\infty$ or a $(d-1)$-dimensional compact subset of $\mathbb{R}^{d}$ with $\overline{\mathcal{M}}^{d-1}(K)<\infty$, then there exists a constant $C$ such that

$$
\mathcal{H}^{d-1}\left(\partial K_{r}\right) \leq C\left(1+r^{d-1}\right), \quad r>0 .
$$


Proof. By Corollary 3.6 in [33], the hypotheses yield $r_{0}, C_{1} \in(0, \infty)$ such that

$$
\mathcal{H}^{d-1}\left(\partial K_{r}\right) \leq C_{1}, \quad r \in\left(0, r_{0}\right) .
$$

Combining Lemma 4.1 of [33] along with Corollaries 2.4 and 2.5 and equation (2.1) of [33], we conclude for almost all $\tilde{r}>0$ that

$$
\mathcal{H}^{d-1}\left(\partial K_{r}\right) \leq(r / \tilde{r})^{d-1} \mathcal{H}^{d-1}\left(\partial K_{\tilde{r}}\right)
$$

for all $r>\tilde{r}$. Choosing such a $\tilde{r}>0$ from $\left(0, r_{0}\right)$ we see that

$$
\mathcal{H}^{d-1}\left(\partial K_{r}\right) \leq C_{1}(1 / \tilde{r})^{d-1} r^{d-1}, \quad r \in\left[r_{0}, \infty\right),
$$

which completes the proof.

Proof OF THEOREM 2.3. Note that we have the same situation as described in Example 1 in Section 2. In the following, we evaluate $I_{K, s}$, which allows us to apply Corollary 2.2. It suffices to show that if $K$ is a full $d$-dimensional subset of $\mathbb{X}$, then $I_{K, s}=O(s)$, while $I_{K, s}=O\left(s^{1-1 / d}\right)$ for lower dimensional $K$. Indeed, put $c:=\min \left\{c_{\mathrm{stab}}, c_{K}\right\} p /\left(36 \cdot 4^{\alpha+1}\right)$, so that

$$
\begin{aligned}
I_{K, s}= & s \int_{\mathbb{X}} \exp \left(-c \mathrm{~d}_{s}(x, K)^{\alpha}\right) g(x) \mathrm{d} x \\
\leq & \|g\|_{\infty} s \int_{K} \exp \left(-c s^{\alpha / d} \mathrm{~d}(x, K)^{\alpha}\right) \mathrm{d} x \\
& +\|g\|_{\infty} s \int_{\mathbb{X} \backslash K} \exp \left(-c s^{\alpha / d} \mathrm{~d}(x, K)^{\alpha}\right) \mathrm{d} x \\
= & \|g\|_{\infty} \operatorname{Vol}_{d}(K) s+\|g\|_{\infty} s \int_{0}^{\infty} \int_{\partial K_{r}} \exp \left(-c s^{\alpha / d} r^{\alpha}\right) \mathcal{H}^{d-1}(\mathrm{~d} y) \mathrm{d} r \\
\leq & \|g\|_{\infty} \operatorname{Vol}_{d}(K) s \\
& +C\left\|_{g}\right\|_{\infty} s \int_{0}^{\infty} \exp \left(-c s^{\alpha / d} r^{\alpha}\right)\left(1+r^{d-1}\right) \mathrm{d} r \\
\leq & \|g\|_{\infty} \operatorname{Vol}_{d}(K) s \\
& +C\|g\|_{\infty} s^{(d-1) / d} \int_{0}^{\infty} \exp \left(-c u^{\alpha}\right)\left(1+s^{-(d-1) / d} u^{d-1}\right) \mathrm{d} u,
\end{aligned}
$$

where the second equality follows by the co-area formula and where the second inequality follows by Lemma 5.12. If $K$ is a full $d$-dimensional subset of $\mathbb{X}$, then the first integral dominates and is $O(s)$. Otherwise, $\operatorname{Vol}_{d}(K)$ vanishes and the second integral is $O\left(s^{(d-1) / d}\right)$. 


\section{APPENDIX}

Here, we provide the proof of Lemma 3.8.

Proof of Lemma 3.8. We need some additional notation. Throughout the proof, $\kappa$ is a constant depending on $d, j$, whose value may change from line to line. For $L$ some linear space, let $\ell^{L}$ the Lebesgue measure on $L, G(L, q), q<\operatorname{dim}(L)$ its space of $q$-dimensional subspaces, and $v_{q}^{L}$ the Haar probability measure on $G(L, q)$. Note $G\left(\mathbb{R}^{d}, j\right)=G(d, j)$ and $v_{j}=v_{j}^{\mathbb{R}^{d}}$. Theorem 6.2.2 from [38] yields

$$
\begin{aligned}
V_{j}(A)-V_{j}(M) & =\kappa \int_{G(d, j)}\left(V_{j}(A \mid L)-V_{j}(M \mid L)\right) v_{j}(\mathrm{~d} L) \\
& =\kappa \int_{G(d, j)} \int_{L} f^{L}(x) \ell^{L}(\mathrm{~d} x) v_{j}(\mathrm{~d} L) .
\end{aligned}
$$

The Blaschke-Petkantschin formula (Theorem 7.2.1 in [38]) over the $\ell^{L}$ integral shows that the right-hand side equals

$$
\kappa \int_{G(d, j)} \int_{G(L, 1)} \int_{N} f^{L}(x)\|x\|^{j-1} \ell^{N}(\mathrm{~d} x) v_{1}^{L}(\mathrm{~d} N) v_{j}(\mathrm{~d} L) .
$$

Fubini's theorem and Theorem 7.1.1 in [38] yield that the last expression is

$$
\begin{aligned}
\kappa \int_{G(d, 1)} & \int_{G(N, j)} \int_{N} f^{L}(x)\|x\|^{j-1} \ell^{N}(\mathrm{~d} x) v_{j}^{N}(\mathrm{~d} L) v_{1}(\mathrm{~d} N) \\
= & \kappa \int_{G(d, 1)} \int_{N} \int_{G(N, j)} f^{L}(x)\|x\|^{j-1} v_{j}^{N}(\mathrm{~d} L) \ell^{N}(\mathrm{~d} x) v_{1}(\mathrm{~d} N) \\
= & \kappa \int_{G(d, 1)} \int_{N}\|x\|^{j-1} \int_{G(L(x), j)} f^{L}(x) v_{j}^{L(x)}(\mathrm{d} L) \ell^{N}(\mathrm{~d} x) v_{1}(\mathrm{~d} N) \\
= & \kappa \int_{G(d, 1)} \int_{N} f(x) \ell^{N}(\mathrm{~d} x) v_{1}(\mathrm{~d} N)
\end{aligned}
$$

with $f(x)=\|x\|^{j-1} \int_{G(L(x), j)} f^{L}(x) v_{j}^{L(x)}(\mathrm{d} L)$ because $N=L(x)$ in the second line. An independent application of the Blaschke-Petkantschin formula with $g(x)=f(x)\|x\|^{-(d-1)}$ for each $L$ yields

$$
\begin{aligned}
\int_{\mathbb{R}^{d}} g(x) \ell^{d}(\mathrm{~d} x) & =\int_{G(d, 1)} \int_{N} g(x)\|x\|^{d-1} \ell^{N}(\mathrm{~d} x) \nu_{1}(\mathrm{~d} N) \\
& =\int_{G(d, 1)} \int_{N} f(x) \ell^{N}(\mathrm{~d} x) \nu_{1}(\mathrm{~d} N)
\end{aligned}
$$

whence (A.1) is equal to $\int_{\mathbb{R}^{d}} \int_{G(L(x), j)} f^{L}(x)\|x\|^{(j-1)-(d-1)} v_{j}^{L(x)}(\mathrm{d} L) \ell^{d}(\mathrm{~d} x)$, which completes the proof. 
Acknowledgments. The authors gratefully acknowledge the comments of an anonymous referee, whose suggestions led to an improved exposition. This research was initiated during the workshop "New Directions in Stein's Method" at the Institute for Mathematical Sciences in Singapore held during May 2015. The authors are grateful to the IMS for their hospitality and support.

\section{REFERENCES}

[1] Avram, F. and Bertsimas, D. (1993). On central limit theorems in geometrical probability. Ann. Appl. Probab. 3 1033-1046. MR1241033

[2] BAI, Z.-D., HWANG, H.-K. and TSAI, T.-H. (2003). Berry-Esseen bounds for the number of maxima in planar regions. Electron. J. Probab. 8 Article ID 9. MR1986841

[3] BÁRÁNY, I., FODOR, F. and VÍGH, V. (2010). Intrinsic volumes of inscribed random polytopes in smooth convex bodies. Adv. in Appl. Probab. 42 605-619. MR2779551

[4] Barbour, A. D. and Xia, A. (2001). The number of two-dimensional maxima. Adv in Appl. Probab. 33 727-750. MR1875775

[5] Barbour, A. D. and XiA, A. (2006). Normal approximation for random sums. Adv in Appl. Probab. 38 693-728. MR2256874

[6] Baryshnikov, Y., Penrose, M. D. and Yukich, J. E. (2009). Gaussian limits for generalized spacings. Ann. Appl. Probab. 19 158-185. MR2498675

[7] BARYSHNIKOV, Y. and YUKICH, J. E. (2005). Gaussian limits for random measures in geometric probability. Ann. Appl. Probab. 15 213-253. MR2115042

[8] Bickel, P. J. and Breiman, L. (1983). Sums of functions of nearest neighbor distances, moment bounds, limit theorems and a goodness of fit test. Ann. Probab. 11 185-214. MR0682809

[9] Calka, P., Schreiber, T. and Yukich, J. E. (2013). Brownian limits, local limits and variance asymptotics for convex hulls in the ball. Ann. Probab. 41 50-108. MR3059193

[10] Chatterjee, S. (2008). A new method of normal approximation. Ann. Probab. 36 15841610. MR2435859

[11] Chen, W.-M., Hwang, H.-K. and Tsai, T.-H. (2003). Efficient maxima-finding algorithms for random planar samples. Discrete Math. Theor. Comput. Sci. 6 107-122. MR2011337

[12] Decreusefond, L., Ferraz, E., Randriambololona, H. and Vergne, A. (2014). Simplicial homology of random configurations. Adv. in Appl. Probab. 46 325-347. MR3215536

[13] Eichelsbacher, P., Raič, M. and Schreiber, T. (2015). Moderate deviations for stabilizing functionals in geometric probability. Ann. Inst. Henri Poincaré B Probab. Stat. 51 89-128. MR3300965

[14] Eichelsbacher, P. and ThäLe, C. (2014). New Berry-Esseen bounds for non-linear functionals of Poisson random measures. Electron. J. Probab. 19 Article ID 102. MR3275854

[15] Goldstein, L., Johnson, T. and Lachièze-Rey, R. (2018). Bounds to the normal for proximity region graphs. Stochastic Process. Appl. 128 1208-1237. MR3769660

[16] Grote, J. and ThäLE, C. (2018). Concentration and moderate deviations for Poisson polytopes and polyhedra. Bernoulli 24 2811-2841. MR3779703

[17] HIRsCH, C. (2017). From heavy-tailed Boolean models to scale-free Gilbert graphs. Braz. J. Probab. Stat. 31 111-143. MR3601663

[18] JimÉnEZ, R. and YUKICH, J. E. (2011). Nonparametric estimation of surface integrals. Ann. Statist. 39 232-260. MR2797845

[19] Kesten, H. and LeE, S. (1996). The central limit theorem for weighted minimal spanning trees on random points. Ann. Appl. Probab. 6 495-527. MR1398055 
[20] LachiÈze-Rey, R. and Peccati, G. (2017). New Berry-Esseen bounds for functionals of binomial point processes. Ann. Appl. Probab. 27 1992-2031. MR3693518

[21] LAChiÈze-ReY, R. and VegA, S. (2017). Boundary density and Voronoi set estimation for irregular sets. Trans. Amer. Math. Soc. 369 4953-4976. MR3632556

[22] Last, G., Peccati, G. and Schulte, M. (2016). Normal approximation on Poisson spaces: Mehler's formula, second order Poincaré inequalities and stabilization. Probab. Theory Related Fields 165 667-723. MR3520016

[23] Last, G. and Penrose, M. D. (2017). Lectures on the Poisson Process. Cambridge Univ. Press, Cambridge.

[24] Penrose, M. (2003). Random Geometric Graphs. Oxford Studies in Probability 5. Oxford Univ. Press, Oxford. MR1986198

[25] Penrose, M. D. (2007). Gaussian limits for random geometric measures. Electron. J. Probab. 12 989-1035. MR2336596

[26] Penrose, M. D. (2007). Laws of large numbers in stochastic geometry with statistical applications. Bernoulli 13 1124-1150. MR2364229

[27] Penrose, M. D. and Rosoman, T. (2008). Error bounds in stochastic-geometrical normal approximation. In Fifth Colloquium on Mathematics and Computer Science. Discrete Math. Theor. Comput. Sci. Proc., AI 71-94. Assoc. Discrete Math. Theor. Comput. Sci., Nancy. MR2508780

[28] Penrose, M. D. and Yukich, J. E. (2001). Central limit theorems for some graphs in computational geometry. Ann. Appl. Probab. 11 1005-1041. MR1878288

[29] Penrose, M. D. and Yukich, J. E. (2003). Weak laws of large numbers in geometric probability. Ann. Appl. Probab. 13 277-303. MR1952000

[30] Penrose, M. D. and Yukich, J. E. (2005). Normal approximation in geometric probability. In Stein's Method and Applications. Lect. Notes Ser. Inst. Math. Sci. Natl. Univ. Singap. 5 37-58. Singapore Univ. Press, Singapore. MR2201885

[31] Penrose, M. D. and Yukich, J. E. (2013). Limit theory for point processes in manifolds. Ann. Appl. Probab. 23 2161-2211. MR3127932

[32] Preparata, F. P. and Shamos, M. I. (1985). Computational Geometry: An Introduction. Texts and Monographs in Computer Science. Springer, New York. MR0805539

[33] RATAJ, J. and WinTER, S. (2010). On volume and surface area of parallel sets. Indiana Univ. Math. J. 59 1661-1685. MR2865426

[34] ReItzner, M. (2005). Central limit theorems for random polytopes. Probab. Theory Related Fields 133 483-507. MR2197111

[35] Reitzner, M. and Schulte, M. (2013). Central limit theorems for $U$-statistics of Poisson point processes. Ann. Probab. 41 3879-3909. MR3161465

[36] RÉNYI, A. and Sulanke, R. (1963). Über die konvexe Hülle von $n$ zufällig gewählten Punkten. Z. Wahrsch. Verw. Gebiete 2 75-84. MR0156262

[37] RÉNYI, A. and Sulanke, R. (1964). Über die konvexe Hülle von $n$ zufällig gewählten Punkten. II. Z. Wahrsch. Verw. Gebiete 3 138-147. MR0169139

[38] SChNeIDER, R. and WeIL, W. (2008). Stochastic and Integral Geometry. Probability and Its Applications (New York). Springer, Berlin. MR2455326

[39] Schoen, R. and YAU, S.-T. (1994). Lectures on Differential Geometry. Conference Proceedings and Lecture Notes in Geometry and Topology. International Press, Cambridge, MA. MR1333601

[40] SChreiber, T. (2010). Limit theorems in stochastic geometry. In New Perspectives in Stochastic Geometry 111-144. Oxford Univ. Press, Oxford. MR2654677

[41] Schreiber, T., Penrose, M. D. and Yukich, J. E. (2007). Gaussian limits for multidimensional random sequential packing at saturation. Comm. Math. Phys. 272 167-183. MR2291806 
[42] Schulte, M. (2012). A central limit theorem for the Poisson-Voronoi approximation. Adv. in Appl. Math. 49 285-306. MR3017961

[43] Schulte, M. (2016). Normal approximation of Poisson functionals in Kolmogorov distance. J. Theoret. Probab. 29 96-117. MR3463079

[44] Sholomov, L. A. (1983). Survey of estimational results in choice problems. Izv. Akad. Nauk SSSR Tekhn. Kibernet. 1 60-87. MR0736267

[45] ThÄLE, C., TURChI, N. and Wespi, F. (2018). Random polytopes: Central limit theorems for intrinsic volumes. Proc. Amer. Math. Soc. 146 3063-3071. MR3787367

[46] THÄLE, C. and YUKICH, J. E. (2016). Asymptotic theory for statistics of the Poisson-Voronoi approximation. Bernoulli 22 2372-2400. MR3498032

[47] Vu, V. (2006). Central limit theorems for random polytopes in a smooth convex set. Adv. Math. 207 221-243. MR2264072

[48] Yukich, J. E. (2015). Surface order scaling in stochastic geometry. Ann. Appl. Probab. 25 177-210. MR3297770

R. LACHIÈZE-REY

LABORATOIRE MAP 5

UNIVERSITÉ PARIS DESCARTES

45 RUE DES SAINTS-PÈRES

75006 PARIS

FRANCE

E-MAIL: raphael.lachieze-rey@parisdescartes.fr
M. SCHULTE

INSTITUT FÜR MATHEMATISCHE STATISTIK UND VERSICHERUNGSLEHRE

UNIVERSITÄT BERN

AlPENEGGSTRASSE 22

3012 BERN

SWITZERLAND

E-MAIL: matthias.schulte@stat.unibe.ch

\author{
J. E. YUKICH \\ DepartMEnT OF MATHEMATICS \\ LEHIGH UNIVERSITY \\ 14 East Packer Ave \\ Bethlehem, PennSylvania 18015 \\ USA \\ E-MAIL: jey0@lehigh.edu
}

